\title{
1 Atmospheric Autoxidation of Organophosphate Esters
}

2 Zihao $\mathrm{Fu}^{\dagger}$, Hong-Bin $\mathrm{Xie}^{\dagger *}$, Jonas Elm ${ }^{\S}$, Yang Liu*, Zhiqiang $\mathrm{Fu}^{\dagger}$, Jingwen Chen ${ }^{\dagger}$

3 †Key Laboratory of Industrial Ecology and Environmental Engineering (Ministry of

4 Education), School of Environmental Science and Technology, Dalian University of

5 Technology, Dalian 116024, China

$6{ }^{\S}$ Department of Chemistry and iClimate, Aarhus University, Langelandsgade 140, DK-

78000 Aarhus C, Denmark

$8 \quad$ School of Chemistry and Chemical Engineering, Harbin Institute of Technology, Harbin

9 150001, China

10

11

Totally, 90 pages, 13 figures and 6 tables 
$13 \mathrm{~S} 1$. Influence of the $-\mathrm{OC}(=\mathrm{O})-,-\mathrm{ONO}_{2}$ and $-\mathrm{OS}(=\mathrm{O})_{2}(\mathrm{OH})$ ester functional groups on the reactivity

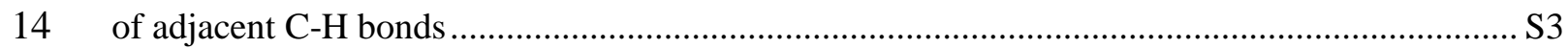

15 S2. The selection of single-point energy calculation method and the extrapolation evaluation ... S5

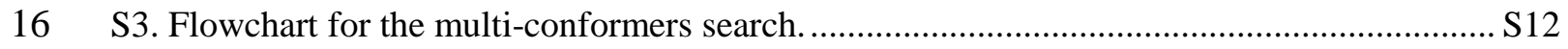

17 S4. Determination of the crucial reaction pathways for multi-conformers calculations ............. S13

18 S5. Calculated Boltzmann populations of the selected reactant conformers and $k_{\mathrm{SC}-\mathrm{TST} i}$ and $k_{\mathrm{MC}-\mathrm{TST}}$

20 S6. Calculated time-dependent fractional yields of the main transformation products from TMP-

$21 \mathrm{R} \cdot+\mathrm{O}_{2}$ and TCPP-R $+\mathrm{O}_{2}$ reactions S29

22 S7. Schematic potential energy surfaces of the various C-center radicals with $\mathrm{O}_{2}$ involved in $\mathrm{R}_{2}$

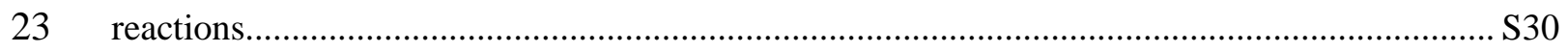

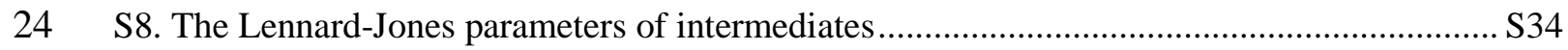

$25 \mathrm{~S} 9$. The effect of the $-(\mathrm{O})_{3} \mathrm{P}(=\mathrm{O})$ functional group on the $\mathrm{H}$-shift reactions ........................... S35

$26 \mathrm{~S} 10$. H-shift reactions of $\mathrm{RO}_{2}$. with the $-(\mathrm{O})_{3} \mathrm{~B},-(\mathrm{O})_{2} \mathrm{~S}(=\mathrm{O})_{2}$ and $-\mathrm{OS}(=\mathrm{O})_{2}(\mathrm{OH})$ ester functional

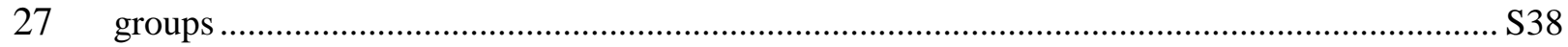

28 S11. Toxicity assessment of products formed in atmospheric transformation of $R_{1}$ and $R_{2} \ldots \ldots . . S 39$

29 S12. Cartesian coordinates of the transition states for all reactions ...................................... S41

30 
32 S1. Influence of the $-\mathrm{OC}(=\mathrm{O})-,-\mathrm{ONO}_{2}$ and $-\mathrm{OS}(=\mathrm{O})_{2}(\mathrm{OH})$ ester functional groups on

33 the reactivity of adjacent $\mathrm{C}-\mathrm{H}$ bonds

34 Previous studies revealed that some carboxylic ethers and organonitrates have lower

35 reaction rate constants than the corresponding alkanes toward $\cdot \mathrm{OH},{ }^{1-3}$ implying that the

36 ester functional groups $-\mathrm{OC}(=\mathrm{O})$ - and $-\mathrm{ONO}_{2}$ could deactivate their adjacent $\mathrm{C}-\mathrm{H}$ bonds to

37 hinder the autoxidation mechanism. Our preliminary studies (see Figure S1) indeed found

38 that the $\mathrm{C}-\mathrm{H}$ bond energies adjacent to $-\mathrm{OC}(=\mathrm{O})$ - and $-\mathrm{ONO}_{2}$ in ethers (by taking methyl

39 acetate $(103.1 \mathrm{kcal} / \mathrm{mol})$ and propyl nitrate $(96.2 \mathrm{kcal} / \mathrm{mol})$ as the model $)$, are higher than

40 the corresponding $\mathrm{C}-\mathrm{H}$ bond energies in alkanes $(98.3 \mathrm{kcal} / \mathrm{mol}$ for propane and 95.2

$41 \mathrm{kcal} / \mathrm{mol}$ for butane), further confirming their deactivating effect on adjacent $\mathrm{C}-\mathrm{H}$ bonds.

42 Similarly, the $\mathrm{C}-\mathrm{H}$ bond energy adjacent to $-\mathrm{OS}(=\mathrm{O})_{2}(\mathrm{OH})$ group in propyl sulfate (95.8

$43 \mathrm{kcal} / \mathrm{mol})$ is not lower than the corresponding $\mathrm{C}-\mathrm{H}$ bond energy in butane $(95.2 \mathrm{kcal} / \mathrm{mol})$,

44 indicating that the $-\mathrm{OS}(=\mathrm{O})_{2}(\mathrm{OH})$ group cannot activate its adjacent $\mathrm{C}-\mathrm{H}$ bonds. More

45 important, high $\mathrm{C}-\mathrm{H}$ bond energy indeed lead to high energy barrier of the H-shift reaction

46 of corresponding $\mathrm{RO}_{2}$ ( (the key step of autoxidation) as shown in Figure $\mathrm{S} 1$, hindering the

47 occurrence of $\mathrm{H}$-shift-driven autoxidation reaction. 


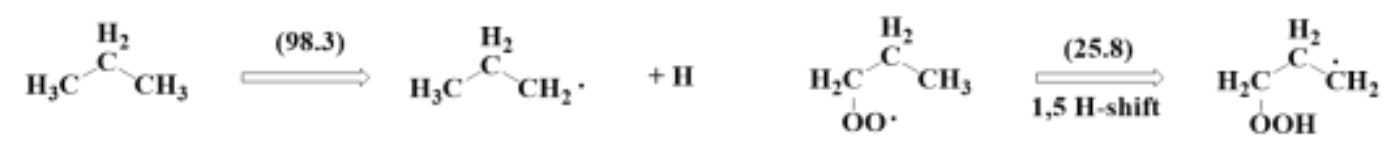

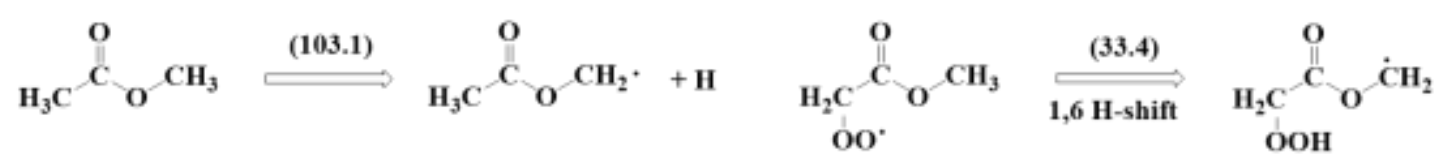

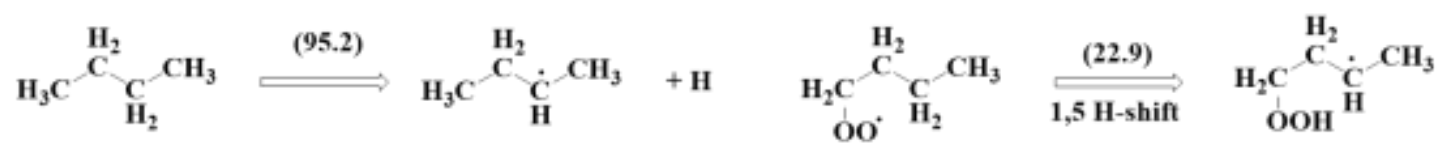

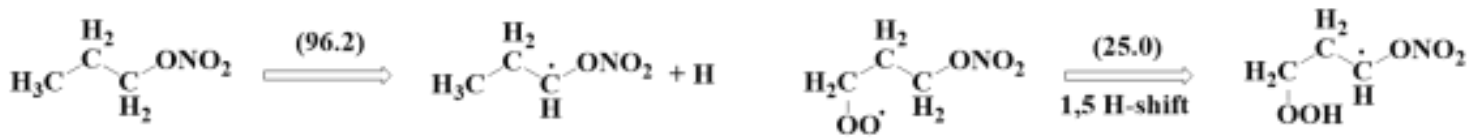
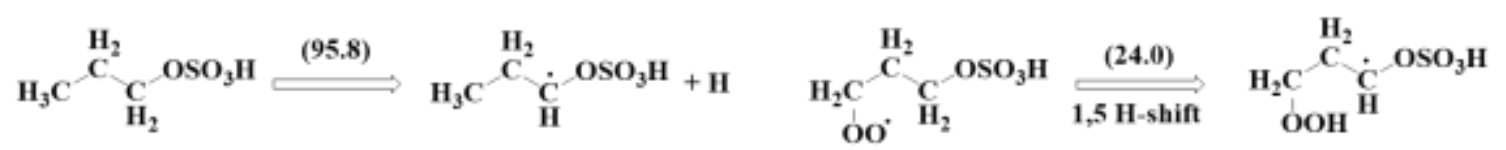

49 Figure S1. Calculated C-H bond energies of propane, butane, methyl acetate, propyl nitrate

50 and propyl sulfate, and $\mathrm{H}$-shift reaction energy barriers of their corresponding $\mathrm{RO}_{2}$. at the

51 M06-2X/6-311+G(3df,2p)//M06-2X/6-31+G(d,p) level of theory. All energetic

52 information is shown in the brackets (in $\mathrm{kcal} \mathrm{mol}^{-1}$ ). 


\section{S2. The selection of single-point energy calculation method and the extrapolation}

\section{5 evaluation}

56 Since the target systems include many atoms, performing a high-level single-point

57 energy (SPE) calculation such as ROHF-ROCCSD(T)-F12a/cc-pVDZ-F12 (abbreviated

$58 \mathrm{~F} 12 \mathrm{a})$ requires highly intensive computation-load, especially for TCPP-RO$\cdot\left(\mathrm{R}_{2}\right)$ system.

59 Here, a benchmark study was performed to identify a reliable and cost-effective SPE

60 method for studying the target systems. In the benchmark study, all six possible

61 unimolecular reaction pathways (as shown in Scheme $\mathrm{S} 1$ ) starting from $\mathrm{R}_{1}$ were selected

62 as test cases.
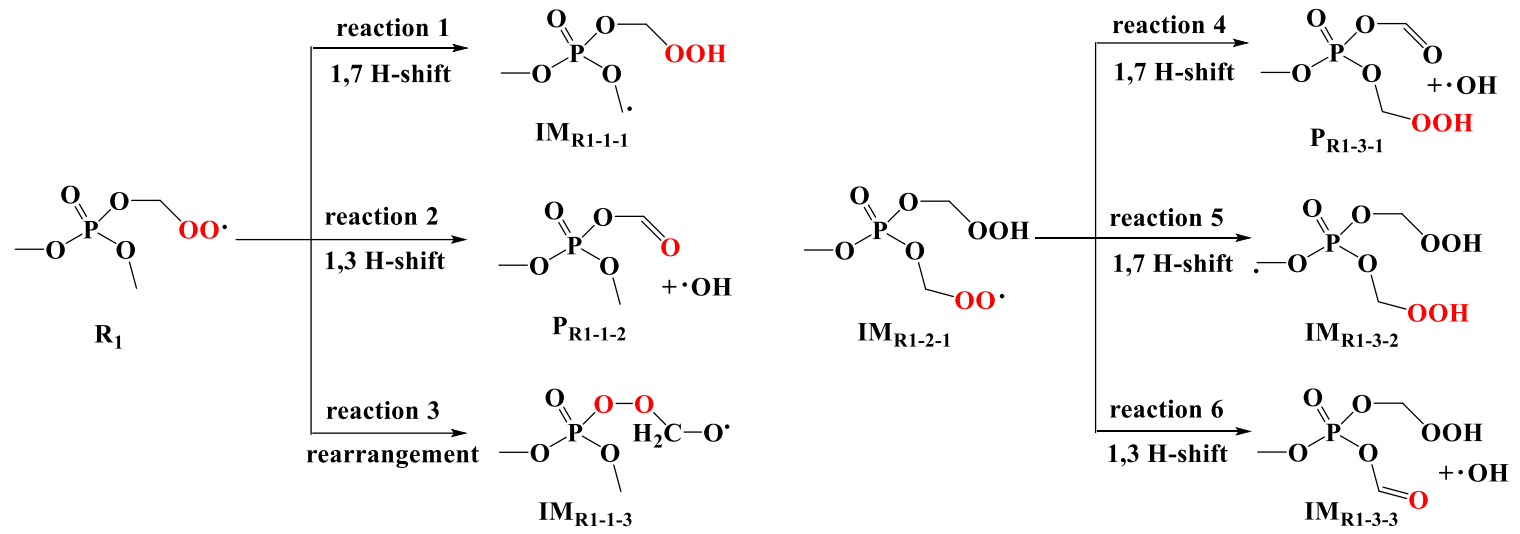

64 Scheme S1. Six possible unimolecular reaction pathways starting from $\mathrm{TMP}-\mathrm{RO}_{2} \cdot\left(\mathrm{R}_{1}\right)$.

$66311+\mathrm{G}(3 \mathrm{df}, 2 \mathrm{p}), \quad$ M06-2X/aug-cc-pVTZ， $\omega B$ 97X-D/aug-cc-pVTZ， ROCBS-QB3， and

67 DLPNO-CCSD(T)/aug-cc-pVTZ have been used to calculate the SPE of all the species

68 involved in these six reactions based on the optimized geometries at the M06-2X/6- 
$6931+\mathrm{G}(\mathrm{d}, \mathrm{p})$ level of theory. Based on the calculated SPE, the reaction energy barriers for

70 these six reactions were calculated by the selected six methods. The mean absolute errors

71 (MAE) of the reaction energy barrier for the five methods M06-2X/6-311+G(3df,2p), M06-

72 2X/aug-cc-pVTZ, $\omega B$ 97X-D/aug-cc-pVTZ, ROCBS-QB3, and DLPNO-CCSD(T)/aug-

73 cc-pVTZ were calculated relative to F12a method. As can be seen in Figure S2, the M06-

$742 \mathrm{X} / 6-311+\mathrm{G}(3 \mathrm{df}, 2 \mathrm{p})$ method exhibits the lowest MAE of $0.37 \mathrm{kcal} \mathrm{mol}^{-1}$ relative to the

75 high level F12a method. If only the H-shift pathways, which are more important than

76 rearrangement pathway for target reactions, were considered, the MAE of M06-2X/6-

$77311+\mathrm{G}(3 \mathrm{df}, 2 \mathrm{p})$ method becomes $0.30 \mathrm{kcal} \mathrm{mol}^{-1}$. Therefore, the M06-2X/6-311+G(3df,2p)

78 method could be a reliable and cost-effective SPE calculation method for the target

79 reactions under study. 

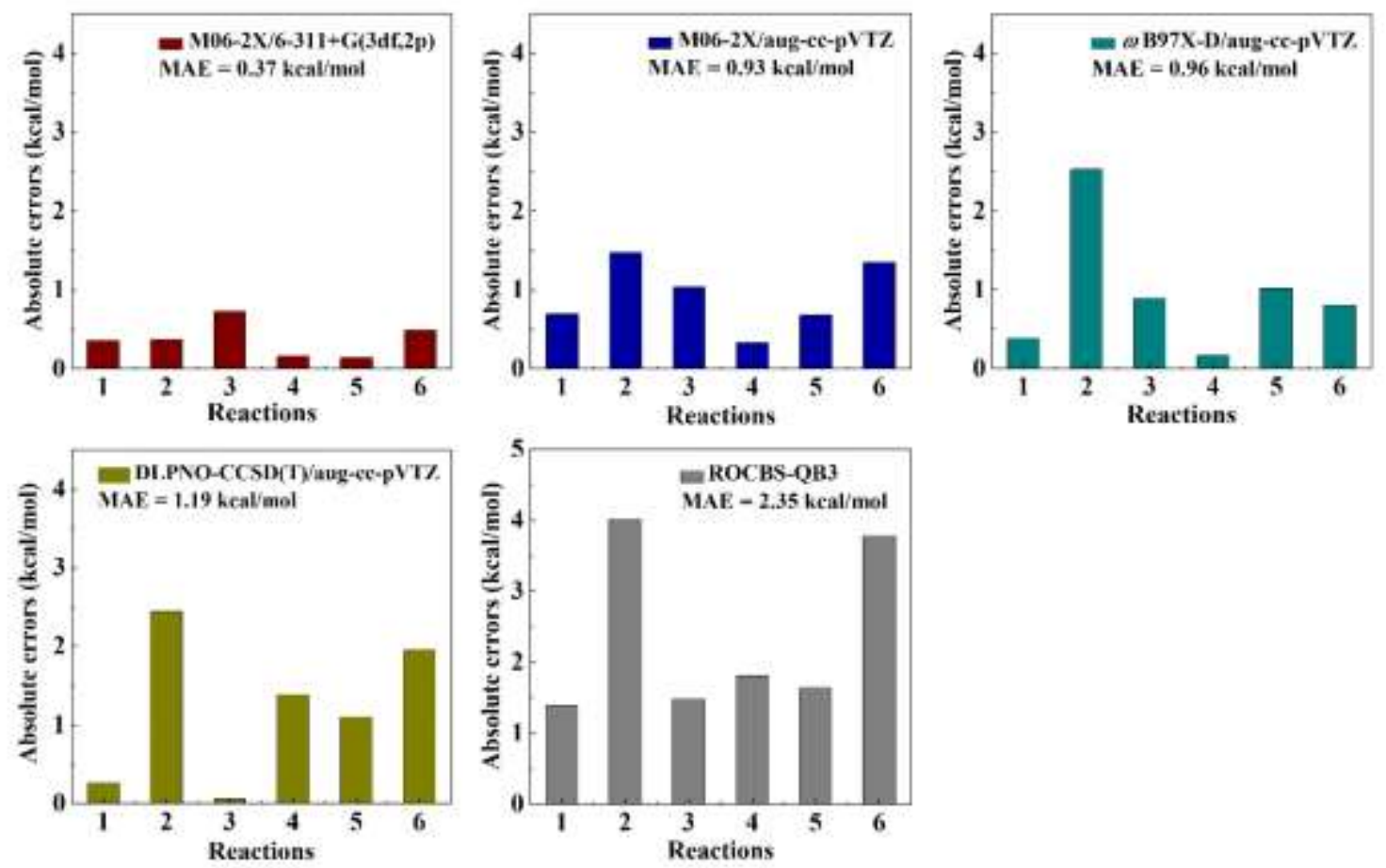

81 Figure S2. Absolute errors of the reaction energy barriers for six representative

82 unimolecular reactions starting from $\mathrm{TMP}^{-\mathrm{RO}_{2}} \cdot\left(\mathrm{R}_{1}\right)$ calculated by M06-2X/6-

83 311+G(3df,2p), M06-2X/aug-cc-pVTZ, $\omega B$ 97X-D/aug-cc-pVTZ，ROCBS-QB3， and

84 DLPNO-CCSD(T)/aug-cc-pVTZ methods, relative to that of high level F12a method. The

85 mean absolute errors (MAE, in $\mathrm{kcal} \mathrm{mol}^{-1}$ ) of the reaction energy barriers produced by the

86 five methods relative to F12a are shown in the corresponding figures.

87 To further evaluate the reliability of M06-2X/6-311+G(3df,2p) in predicting the

88 barrier heights for $R_{2}$ system, the barrier heights from M06-2X/6-311+G(3df,2p) method

89 for selected test reaction systems are compared with those from an average scheme of

90 multiple methods (eq.1). 


$$
\begin{aligned}
& E_{\mathrm{R} 2 \text { reaction } j}(\text { average scheme })=\frac{1}{4}\left(E_{\mathrm{R}_{2} \text { reaction } j}(\mathrm{M} 06-2 \mathrm{X} / 6-311+\mathrm{G}(3 \mathrm{df}, 2 \mathrm{p}))+\right. \\
& E_{\mathrm{R} 2_{2} \text { reaction } j}(\mathrm{M} 06-2 \mathrm{X} / \text { aug-cc-pVTZ })+ \\
& E_{\mathrm{R} 2 \text { reaction } j}(\omega \mathrm{B} 97 \mathrm{X}-\mathrm{D} / \text { aug-cc-pVTZ })+ \\
& \text { eq. } 1 \\
& \left.E_{\mathrm{R}_{2} \text { reaction } j}(\mathrm{DLPNO}-\mathrm{CCSDT} / \text { aug-cc-pVTZ })\right)+ \\
& \mathrm{MAE}_{\mathrm{R} 1}
\end{aligned}
$$

92 where the $E_{\mathrm{R} 2 \text { reaction } j}$ (method) represents the reaction energy barrier of selected reactions

$93 j$ of $\mathrm{R}_{2}$ calculated by selected four methods; the $\mathrm{MAE}_{\mathrm{R} 1}$ is the mean absolute error of the

94 selected four methods for calculating reaction energy barriers of six reactions for $\mathrm{R}_{1}$ system.

95 The $\mathrm{MAE}_{\mathrm{R} 1}$ can be calculated by the following eq.2:

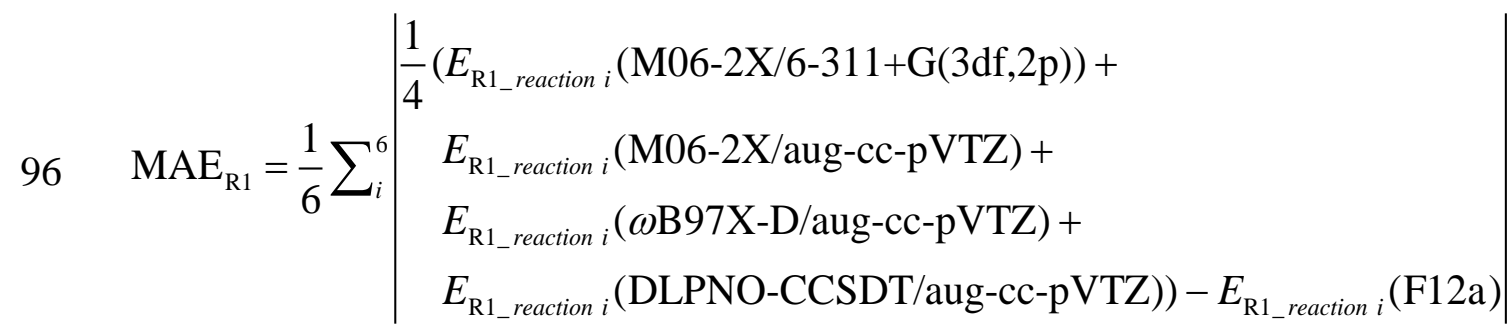
eq. 2

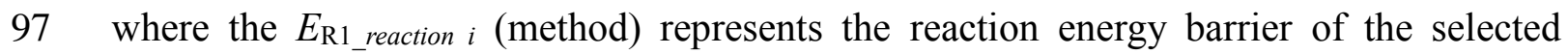

98 reaction $i$ of $\mathrm{R}_{1}$ calculated by the selected four methods and high level F12a method. Here,

99 we assumed that the average scheme of multiple methods can produce a more reliable

100 barrier height, compared to a single method. A previous study also found that an average

101 scheme of different functionals can produce a better result since the average of different

102 methods could cancel potential errors arising from each method. ${ }^{4}$ The test reaction systems

103 come from five key reaction pathways of $\mathrm{R}_{2}$, and two conformers are selected for each key

104 reaction pathway. Therefore, totally 10 reactions were selected in the test reaction systems

105 (see Scheme S2). The top best four methods (based on the calculated MAE for $\mathrm{R}_{1}$ reactions) 
107 and DLPNO-CCSD(T)/aug-cc-pVTZ are selected as multiple methods (as shown in Figure

108 S2).

109

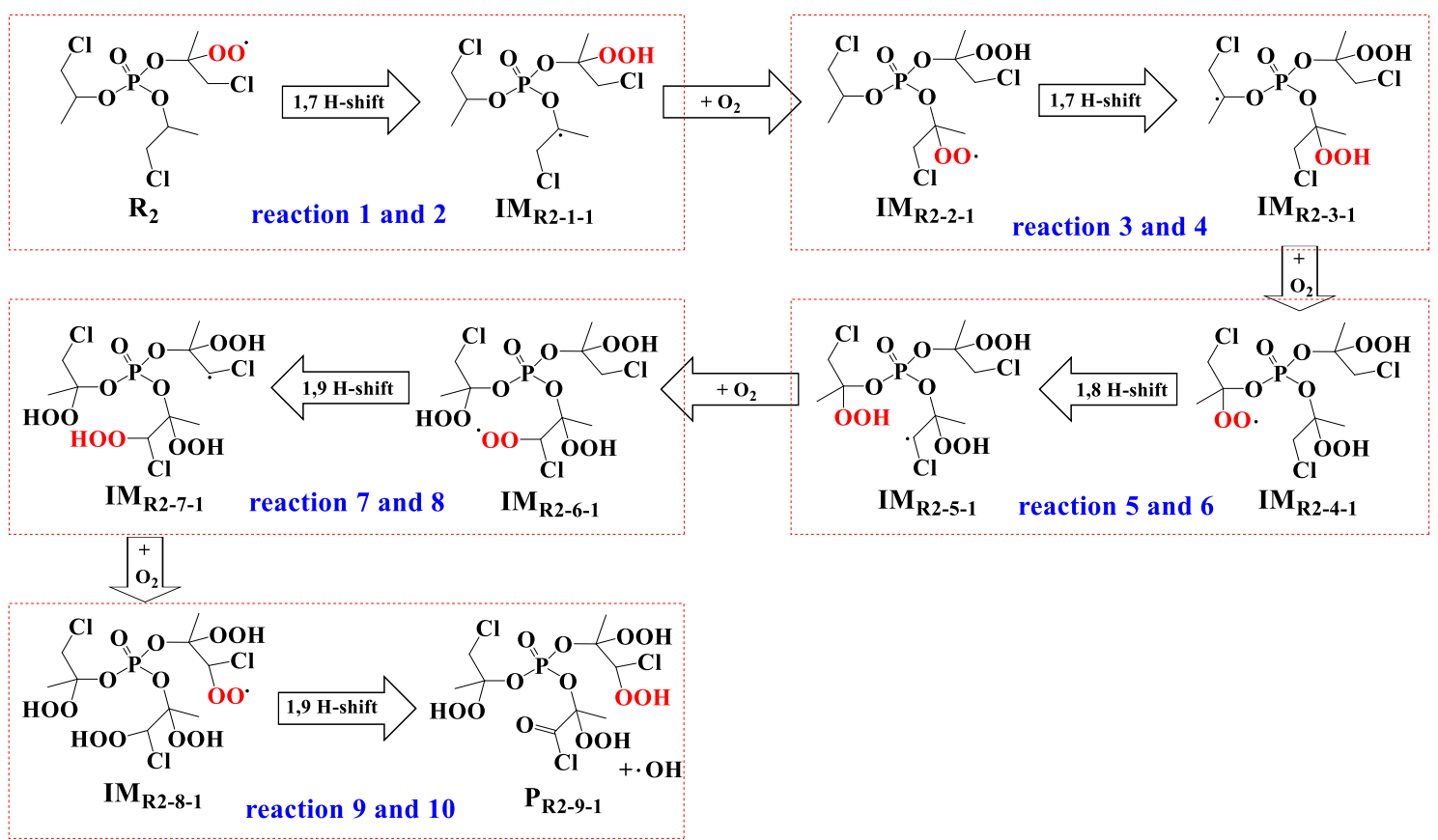

110 Scheme $\mathrm{S} 2$. Selected ten test H-shift reactions of TCPP-RO ${ }_{2} \cdot\left(\mathrm{R}_{2}\right)$ system.

111 As shown in Figure S3, the calculated barrier heights for selected key reaction

112 pathways of TCPP- $\mathrm{RO}_{2} \cdot$ system based on this scheme are very close to corresponding

113 values at M06-2X/6-311+G(3df,2p) level of theory with a MAE of $0.29 \mathrm{kcal} \mathrm{mol}^{-1}$. Such

114 consistence in predicting barrier heights between the average scheme of multiple methods

115 and M06-2X/6-311+G(3df,2p) could further indicate that M06-2X/6-311+G(3df,2p) can

116 well predict the barrier heights of TCPP- $\mathrm{RO}_{2} \cdot$ reaction system. 


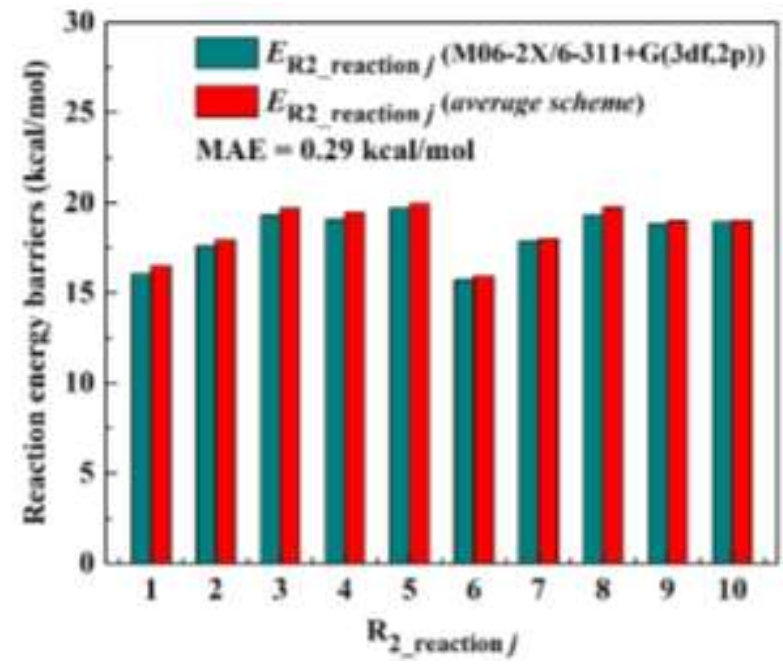

118 Figure S3. The reaction energy barriers for ten representative unimolecular reactions

119 starting from TCPP-RO ${ }_{2} \cdot\left(\mathrm{R}_{2}\right)$ calculated by M06-2X/6-311+G(3df,2p) method and an

120 average scheme of multiple methods. The mean absolute errors (MAE, in $\mathrm{kcal} \mathrm{mol}^{-1}$ ) of

121 the reaction energy barriers produced by the average scheme relative to M06-2X/6-

$122311+\mathrm{G}(3 \mathrm{df}, 2 \mathrm{p})$ is shown in the figure.

123 It is found that the M06-2X with two different basis sets (6-311+G(3df,2p) and aug-

124 cc-pVTZ) produce a relatively large difference in the reaction energy barriers. The M06-

$1252 \mathrm{X}$ with 6-311+G(3df,2p) gives a smaller MAE, compared to that with aug-cc-pVTZ. We

126 further tested whether the difference still exist when other functionals were employed. It

127 was found that the relatively large difference in the reaction energy barrier exist when other

128 functionals such as $\omega$ B97X-D and B3LYP with 6-311+G(3df,2p) and aug-cc-pVTZ were

129 employed (see Figure S4). However, the 6-311+G(3df,2p) does not always give a smaller

130 MAE when the functional changes, compared to aug-cc-pVTZ, e.g. wB97X-D with 6- 
$131311+\mathrm{G}(3 \mathrm{df}, 2 \mathrm{p})$ gives a greater MAE, compared to that with aug-cc-pVTZ. In addition, as

132 shown in Figure S4, it was found that MAE changes little when M06-2X was used and 6-

$133311+\mathrm{G}(3 \mathrm{df}, 2 \mathrm{p})$ was changed to $6-311+\mathrm{G}(3 \mathrm{df}, 2 \mathrm{pd})$ and $6-311+\mathrm{G}(3 \mathrm{df}, 3 \mathrm{pd})$. Hence, the 6-

$134311+\mathrm{G}(3 \mathrm{df}, 2 \mathrm{p})$ basis set can be expected to yield accurate reaction energy barriers for the

135 studied systems, due to a favorable, and consistent, cancellation of errors.
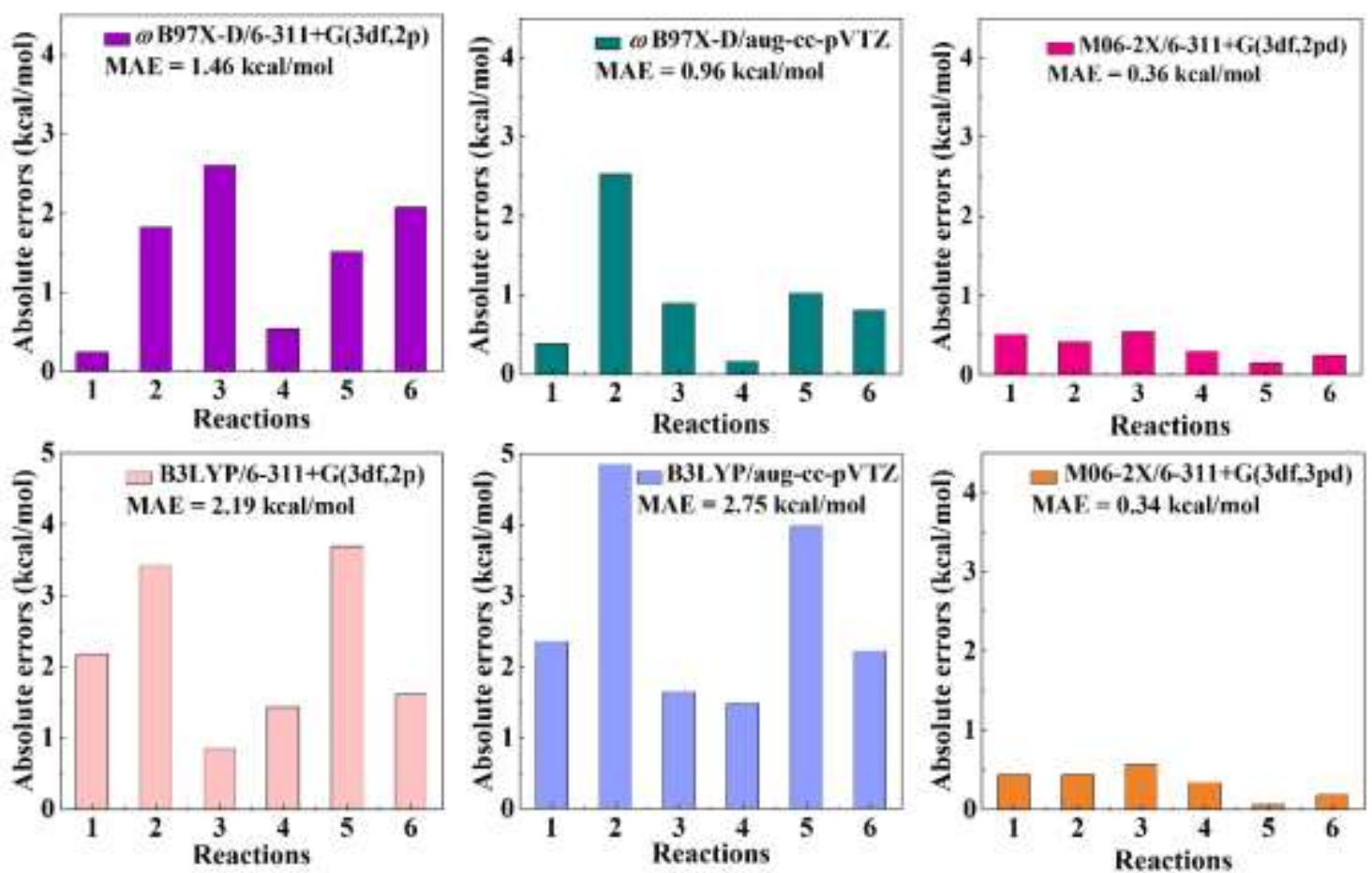

137 Figure S4. Absolute errors of the reaction energy barriers for six representative 138 unimolecular reactions starting from $\mathrm{TMP}-\mathrm{RO}_{2} \cdot\left(\mathrm{R}_{1}\right)$ calculated by $\omega \mathrm{B} 97 \mathrm{X}-\mathrm{D} / 6-$ $139311+\mathrm{G}(3 \mathrm{df}, 2 \mathrm{p}), \omega \mathrm{B} 97 \mathrm{X}-\mathrm{D} /$ aug-cc-pVTZ, B3LYP/6-311+G(3df,2p), B3LYP/aug-cc-pVTZ, 140 M06-2X/6-311+G(3df,2pd), and M06-2X/6-311+G(3df,3pd) methods, relative to that of 141 high level F12a method. The mean absolute errors (MAE, in $\mathrm{kcal} \mathrm{mol}^{-1}$ ) of the reaction 
142 energy barriers produced by the six methods relative to F12a are shown in the

143 corresponding figures.

144 S3. Flowchart for the multi-conformers search.

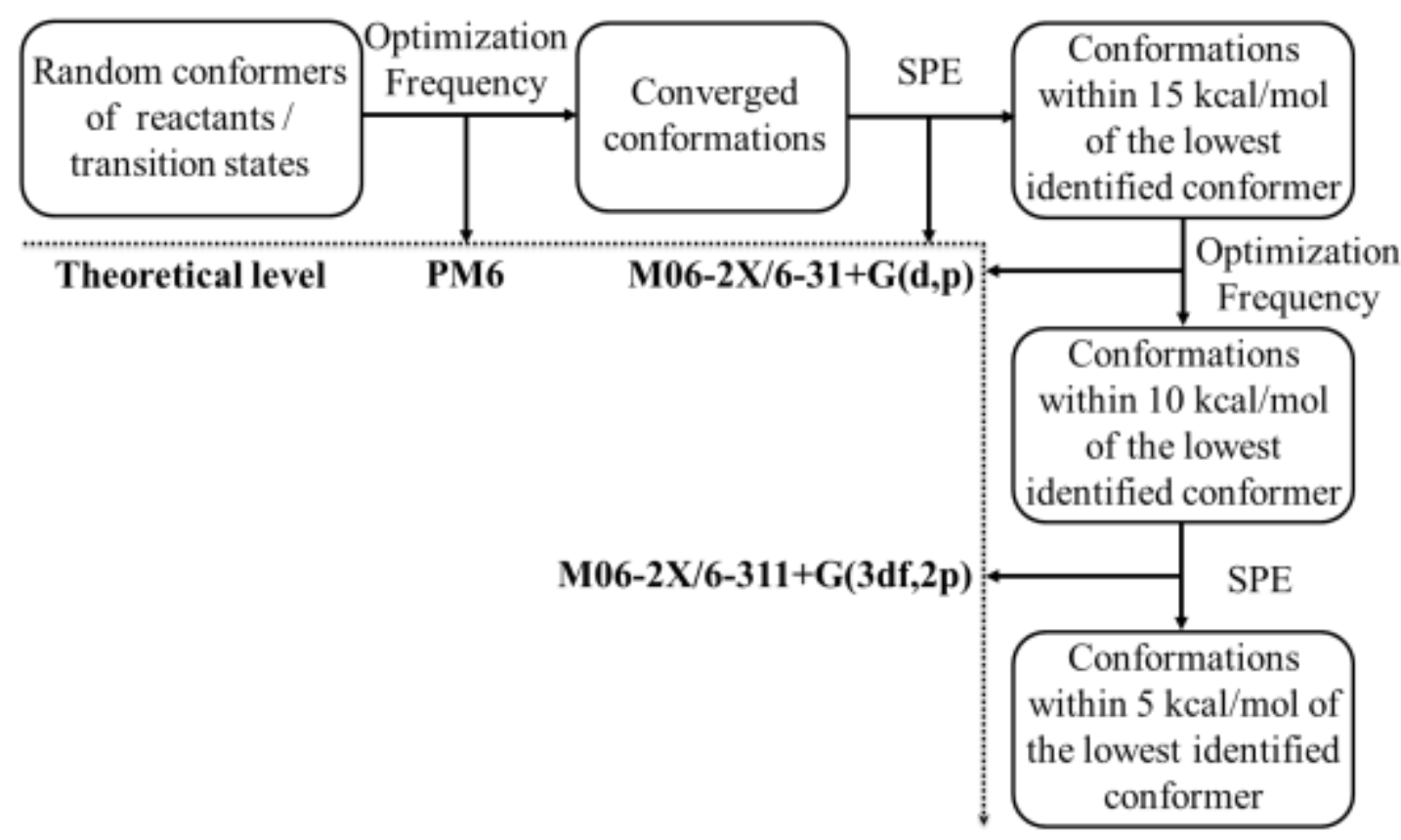

146 Figure S5. Flowchart for the multi-conformers search. "SPE" represents the single point

147 energy calculation. All optimizations, frequency, and SPE calculations with semi-empirical

148 PM6 level or density functional theory (M06-2X functional) were performed in Gaussian

14909 program. Unique conformers are identified based on their calculated energies and

150 distance matrix using the script isostat in Molclus program. 
151 S4. Determination of the crucial reaction pathways for multi-conformers calculations

152 The potential crucial reaction pathways in this study include the H-shift reactions of

153 all formed peroxy radicals derived from TMP- $\mathrm{RO}_{2} \cdot\left(\mathrm{R}_{1}\right)$ and TCPP- $\mathrm{RO}_{2} \cdot\left(\mathrm{R}_{2}\right)$ under the

154 low NO conditions, as well as the dissociation reactions of TMP-RO and TCPP-RO under

155 the high $\mathrm{NO}$ conditions. In the following part, taking the $\mathrm{R}_{2}$ reaction as an example to

156 illustrate how to determine the crucial reaction pathways for multi-conformers calculation.

$157 \mathrm{R}_{2} \rightarrow \mathrm{IM}_{\mathrm{R} 2-1-1} \rightarrow \mathrm{IM}_{\mathrm{R} 2-2-1}$ is determined to be the most favorable unimolecular reaction

158 pathways, in terms of the schematic potential energy surfaces and corresponding kinetics

159 analysis from single-conformer approximation at the M06-2X/6-311+G(3df,2p)//M06-

$1602 \mathrm{X} / 6-31+\mathrm{G}(\mathrm{d}, \mathrm{p})$ level. The high-pressure limit reaction rate constant for $\mathrm{R}_{2} \rightarrow \mathrm{IM}_{\mathrm{R} 2-1-1}$ is

161 calculated to be $13.9 \mathrm{~s}^{-1}$, about two orders of magnitude higher than the reaction rate

162 constants of $\mathrm{R}_{2}$ reaction with $100 \mathrm{ppt} \mathrm{NO}\left(0.023 \mathrm{~s}^{-1}\right)$ and $40 \mathrm{ppt} \mathrm{HO}_{2} \cdot\left(0.017 \mathrm{~s}^{-1}\right)$, based on

163 the fixed reaction rate constants for the bimolecular reactions of peroxy radicals with

$164 \mathrm{NO} / \mathrm{HO}_{2} \cdot\left(k_{\mathrm{RO} 2+\mathrm{NO}}=9.0 \times 10^{-12} \mathrm{~cm}^{3}\right.$ molecule $^{-1} \mathrm{~s}^{-1}$ and $k_{\mathrm{RO} 2+\mathrm{HO} 2}=1.7 \times 10^{-11} \mathrm{~cm}^{3}$

165 molecule $\left.{ }^{-1} \mathrm{~s}^{-1}\right) \cdot{ }^{5,6}$ The two orders of magnitude difference in reaction rate constants could

166 still be a possible computational error range caused by single-conformer approximation

167 calculation. Therefore, $\mathrm{R}_{2} \rightarrow \mathrm{IM}_{\mathrm{R} 2-1-1}$ is selected as one crucial pathway for $\mathrm{R}_{2}$ reaction. In

168 addition, the high-pressure limit reaction rate constant for $\operatorname{IM}_{\mathrm{R} 2-1-1} \rightarrow \mathrm{R}_{2}\left(8.3 \times 10^{4} \mathrm{~s}^{-1}\right)$

169 could be comparable to the reaction rate constant $\left(3.0 \times 10^{7} \mathrm{~s}^{-1}\right)$ of $\mathrm{IM}_{\mathrm{R} 2-1-1}$ with $\mathrm{O}_{2}$ (based 
170 on the reaction rate constant $\left(6 \times 10^{-12} \mathrm{~cm}^{3}\right.$ molecule $\left.{ }^{-1} \mathrm{~s}^{-1}\right)$ of $\mathrm{C}$-centered radical with $\mathrm{O}_{2}$

171 and the atmospheric concentration of $\mathrm{O}_{2}\left(5.0 \times 10^{18}\right.$ molecule $\left.\left.\mathrm{cm}^{-3}\right)\right)$ within the range of

172 the possible computational error. Therefore, $\mathrm{IM}_{\mathrm{R} 2-1-1} \rightarrow \mathrm{R}_{2}$ is also selected as one crucial

173 pathway for $\mathrm{R}_{2}$ reaction. To further consider the effect of possible computational error on

174 the competition of unimolecular reaction of $R_{2}$, reaction pathway $R_{2} \leftrightarrow I_{R 2-1-2}$, which

175 reaction energy barrier is the closest to that of $\mathrm{R}_{2} \leftrightarrow \mathrm{IM}_{\mathrm{R} 2-1-1}$, is also selected as one crucial

176 pathway for the unimolecular reaction of $R_{2}$. All in all, $R_{2} \leftrightarrow I_{R 2-1-1}$ and $R_{2} \leftrightarrow I_{R 2-1-2}$

177 are determined as the final crucial reaction pathways for the unimolecular reactions of $\mathrm{R}_{2}$.

178 Based on the same principle, the reaction pathways $\mathrm{IM}_{\mathrm{R} 2-2-1} \leftrightarrow \mathrm{IM}_{\mathrm{R} 2-3-1}$ and $\mathrm{IM}_{\mathrm{R} 2-2-1} \leftrightarrow$

$179 \mathrm{IM}_{\mathrm{R} 2-3-2} ; \mathrm{IM}_{\mathrm{R} 2-4-1} \leftrightarrow \mathrm{IM}_{\mathrm{R} 2-5-1}$ and $\mathrm{IM}_{\mathrm{R} 2-4-1} \leftrightarrow \mathrm{IM}_{\mathrm{R} 2-5-2} ; \mathrm{IM}_{\mathrm{R} 2-6-1} \leftrightarrow \mathrm{IM}_{\mathrm{R} 2-7-1}$ and $\mathrm{IM}_{\mathrm{R} 2-6-1} \leftrightarrow$

$180 \quad \mathrm{IM}_{\mathrm{R} 2-7-2} ; \mathrm{IM}_{\mathrm{R} 2-8-1} \rightarrow \mathrm{P}_{\mathrm{R} 2-9-1}$ and $\mathrm{IM}_{\mathrm{R} 2-8-1} \rightarrow \mathrm{P}_{\mathrm{R} 2-9-2}$ are selected as crucial ones for the $\mathrm{R}_{2}$

181 reactions. Similarly, the reaction pathways $\mathrm{R}_{1} \leftrightarrow \mathrm{IM}_{\mathrm{R} 1-1-1} ; \mathrm{IM}_{\mathrm{R} 1-2-1} \rightarrow \mathrm{P}_{\mathrm{R} 1-3-1}$ and $\mathrm{IM}_{\mathrm{R} 1-2-1} \leftrightarrow$

$182 \mathrm{P}_{\mathrm{R} 1-3-2}$ are selected as crucial ones for the $\mathrm{R}_{1}$ reactions. In addition, the reaction pathways

$183 \mathrm{TMP}-\mathrm{RO} \cdot \rightarrow \mathrm{P}_{\mathrm{R} 1-4-1}$ and TMP-RO $\rightarrow \mathrm{P}_{\mathrm{R} 1-4-2}$; TCPP-RO $\rightarrow \mathrm{P}_{\mathrm{R} 1-10-1}$ and TCPP-RO $\rightarrow \mathrm{P}_{\mathrm{R} 1-}$

184 10-2 are selected as crucial ones for the reactions of TMP-RO· and TCPP-RO·, respectively.

185 The applied reaction rate constants for these selected reaction pathways are presented in

186 Table S1. 
187 Table S1. Calculated high-pressure limit reaction rate constants $(k)$ for the crucial reaction 188 pathways in the reaction systems $R_{1}$ and $R_{2}$ based on the energetics data from single189 conformer approximation and at M06-2X/6-311+G(3df,2p)//M06-2X/6-31+G(d,p) level of 190 theory.

\begin{tabular}{cc}
\hline Reactions & $k\left(\mathrm{~s}^{-1}\right)$ \\
\hline $\mathrm{R}_{1} \rightarrow \mathrm{IM}_{\mathrm{R} 1-1-1}$ & 0.43 \\
$\mathrm{IM}_{\mathrm{R} 1-1-1} \rightarrow \mathrm{R}_{1}$ & $3.4 \times 10^{5}$ \\
$\mathrm{IM}_{\mathrm{R} 1-2-1} \rightarrow \mathrm{P}_{\mathrm{R} 1-3-1}$ & 3.8 \\
$\mathrm{IM}_{\mathrm{R} 1-2-1} \rightarrow \mathrm{P}_{\mathrm{R} 1-3-2}$ & 0.10 \\
$\mathrm{IM}_{\mathrm{R} 1-3-2} \rightarrow \mathrm{P}_{\mathrm{R} 1-2-1}$ & $3.7 \times 10^{7}$ \\
$\mathrm{TMP}-\mathrm{RO} \rightarrow \mathrm{P}_{\mathrm{R} 1-4-1}$ & $5.4 \times 10^{6}$ \\
$\mathrm{TMP}-\mathrm{RO} \rightarrow \mathrm{P}_{\mathrm{R} 1-4-2}$ & $3.5 \times 10^{2}$ \\
$\mathrm{R}_{2} \rightarrow \mathrm{I} \mathrm{M}_{\mathrm{R} 2-1-1}$ & 13.9 \\
$\mathrm{IM}_{\mathrm{R} 2-1-1} \rightarrow \mathrm{R}_{2}$ & $8.3 \times 10^{4}$ \\
$\mathrm{R}_{2} \rightarrow \mathrm{I} \mathrm{M}_{\mathrm{R} 2-1-2}$ & 1.0 \\
$\mathrm{IM}_{\mathrm{R} 2-1-2} \rightarrow \mathrm{R}_{2}$ & $2.1 \times 10^{7}$ \\
$\mathrm{IM}_{\mathrm{R} 2-2-1} \rightarrow \mathrm{IM}_{\mathrm{R} 2-3-1}$ & 19.1 \\
$\mathrm{IM}_{\mathrm{R} 2-3-1} \rightarrow \mathrm{IM}_{\mathrm{R} 2-2-1}$ & $1.5 \times 10^{5}$ \\
$\mathrm{IM}_{\mathrm{R} 2-2-1} \rightarrow \mathrm{IM}_{\mathrm{R} 2-3-2}$ & 0.60 \\
$\mathrm{IM}_{\mathrm{R} 2-3-2} \rightarrow \mathrm{IM}_{\mathrm{R} 2-2-1}$ & $2.1 \times 10^{7}$ \\
$\mathrm{IM}_{\mathrm{R} 2-4-1} \rightarrow \mathrm{IM}_{\mathrm{R} 2-5-1}$ & 3.0 \\
$\mathrm{IM}_{\mathrm{R} 2-5-1} \rightarrow \mathrm{IM}_{\mathrm{R} 2-4-1}$ & $7.0 \times 10^{4}$ \\
$\mathrm{IM}_{\mathrm{R} 2-4-1} \rightarrow \mathrm{IM}_{\mathrm{R} 2-5-2}$ & 0.39 \\
$\mathrm{IM}_{\mathrm{R} 2-5-2} \rightarrow \mathrm{IM}_{\mathrm{R} 2-4-1}$ & $4.9 \times 10^{3}$ \\
$\mathrm{IM}_{\mathrm{R} 2-6-1} \rightarrow \mathrm{IM}_{\mathrm{R} 2-7-1}$ & 1.4 \\
$\mathrm{IM}_{\mathrm{R} 2-7-1} \rightarrow \mathrm{IM}_{\mathrm{R} 2-6-1}$ & $1.4 \times 10^{4}$ \\
$\mathrm{IM}_{\mathrm{R} 2-6-1} \rightarrow \mathrm{IM}_{\mathrm{R} 2-7-2}$ & $3.8 \times 10^{-4}$ \\
$\mathrm{IM}_{\mathrm{R} 2-7-2} \rightarrow \mathrm{IM}_{\mathrm{R} 2-6-1}$ & $4.5 \times 10^{5}$ \\
$\mathrm{IM}_{\mathrm{R} 2-8-1} \rightarrow \mathrm{P}_{\mathrm{R} 2-9-1}$ & 0.78 \\
$\mathrm{IM}_{\mathrm{R} 2-8-1} \rightarrow \mathrm{P}_{\mathrm{R} 2-9-2}$ & $4.3 \times 10^{-5}$ \\
$\mathrm{TCPP} \mathrm{T}_{\mathrm{R} 1-10-1} \rightarrow \mathrm{P}_{\mathrm{R}}$ & $2.3 \times 10^{7}$ \\
$\mathrm{TCPP}-\mathrm{RO} \rightarrow \mathrm{P}_{\mathrm{R} 1-10-2}$ & $2.6 \times 10^{3}$ \\
\hline & \\
\hline & \\
\hline
\end{tabular}

191 
192 S5. Calculated Boltzmann populations of the selected reactant conformers and $\boldsymbol{k}_{\mathrm{SC}}$ -

\section{$193 \quad$ TST $i$ and $k_{\text {MC-TST }}$}

194 Table S2. Calculated relative free energy $\left(\Delta G_{i}\right.$, the free energy of global minimum is set as

195 a reference state for each species) and Boltzmann populations ( $\left.w_{i}\right)$ of the selected reactant

196 conformers at the M06-2X/6-311+G(3df,2p)//M06-2X/6-31+G(d,p) level of theory.

\begin{tabular}{lcc}
\hline Species & $\Delta G_{i}\left(\mathrm{kcal} \mathrm{mol}^{-1}\right)$ & $w_{i}$ \\
\hline $\mathrm{R}_{1-1}$ & 0.0000 & 0.1806 \\
$\mathrm{R}_{1}-2$ & 0.0703 & 0.1604 \\
$\mathrm{R}_{1-3}$ & 0.1445 & 0.1415 \\
$\mathrm{R}_{1}-4$ & 0.2008 & 0.1286 \\
$\mathrm{R}_{1-5}$ & 0.3232 & 0.1046 \\
$\mathrm{R}_{1-6}$ & 0.6003 & 0.0655 \\
$\mathrm{R}_{1-7}$ & 0.6546 & 0.0598 \\
$\mathrm{R}_{1-8}$ & 0.7649 & 0.0496 \\
$\mathrm{R}_{1-9}$ & 0.8421 & 0.0435 \\
$\mathrm{R}_{1-10}$ & 1.1606 & 0.0254 \\
$\mathrm{R}_{1-11}$ & 1.3207 & 0.0194 \\
$\mathrm{R}_{1-12}$ & 1.4888 & 0.0146 \\
$\mathrm{R}_{1-13}$ & 2.4232 & 0.0030 \\
$\mathrm{R}_{1-14}$ & 2.6673 & 0.0020 \\
$\mathrm{R}_{1-15}$ & 2.8491 & 0.0015 \\
\hline $\mathrm{IM}_{\mathrm{R} 1-1-1}-1$ & 0.0000 & 0.5784 \\
$\mathrm{IM}_{\mathrm{R} 1-1-1}-2$ & 0.7506 & 0.1627 \\
$\mathrm{IM}_{\mathrm{R} 1-1-1}-3$ & 1.3121 & 0.0630 \\
$\mathrm{IM}_{\mathrm{R} 1-1-1}-4$ & 1.5578 & 0.0416 \\
$\mathrm{IM}_{\mathrm{R} 1-1-1}-5$ & 1.6197 & 0.0375 \\
$\mathrm{IM}_{\mathrm{R} 1-1-1}-6$ & 1.7408 & 0.0305 \\
$\mathrm{IM}_{\mathrm{R} 1-1-1}-7$ & 2.0973 & 0.0167 \\
$\mathrm{IM}_{\mathrm{R} 1-1-1}-8$ & 2.2134 & 0.0137 \\
$\mathrm{IM}_{\mathrm{R} 1-1-1}-9$ & 2.2514 & 0.0129 \\
$\mathrm{IM}_{\mathrm{R} 1-1-1}-10$ & 2.2632 & 0.0126 \\
$\mathrm{IM}_{\mathrm{R} 1-1-1}-11$ & 2.2877 & 0.0121 \\
$\mathrm{IM}_{\mathrm{R} 1-1-1}-12$ & 2.4251 & 0.0096 \\
& $\mathrm{~S} 16$ & \\
& &
\end{tabular}




\begin{tabular}{|c|c|c|}
\hline $\mathrm{IM}_{\mathrm{R} 1-1-1-13}$ & 2.9402 & 0.0040 \\
\hline $\mathrm{IM}_{\mathrm{R} 1-1-1-14}$ & 3.0282 & 0.0035 \\
\hline $\mathrm{IM}_{\mathrm{R} 1-1-1-15}$ & 3.8624 & 0.0008 \\
\hline $\mathrm{IM}_{\mathrm{R} 1-1-1}-16$ & 4.8494 & 0.0002 \\
\hline $\mathrm{IM}_{\mathrm{R} 1-2-1-1}$ & 0.0000 & 0.3110 \\
\hline $\mathrm{IM}_{\mathrm{R} 1-2-1-2}$ & 0.4506 & 0.1453 \\
\hline $\mathrm{IM}_{\mathrm{R} 1-2-1-3}$ & 0.5440 & 0.1241 \\
\hline $\mathrm{IM}_{\mathrm{R} 1-2-1-4}$ & 0.6265 & 0.1079 \\
\hline $\mathrm{IM}_{\mathrm{R} 1-2-1-5}$ & 0.8400 & 0.0752 \\
\hline$I_{\mathrm{R} 1-2-1-6}$ & 0.8958 & 0.0685 \\
\hline $\mathrm{IM}_{\mathrm{R} 1-2-1-7}$ & 1.1226 & 0.0467 \\
\hline $\mathrm{IM}_{\mathrm{R} 1-2-1-8}$ & 1.1782 & 0.0425 \\
\hline $\mathrm{IM}_{\mathrm{R} 1-2-1-9}$ & 1.2398 & 0.0383 \\
\hline $\mathrm{IM}_{\mathrm{R} 1-2-1-10}$ & 1.5825 & 0.0215 \\
\hline $\mathrm{IM}_{\mathrm{R} 1-2-1-11}$ & 1.8167 & 0.0144 \\
\hline $\mathrm{IM}_{\mathrm{R} 1-2-1-12}$ & 2.8373 & 0.0026 \\
\hline $\mathrm{IM}_{\mathrm{R} 1-2-1-13}$ & 3.2755 & 0.0012 \\
\hline $\mathrm{IM}_{\mathrm{R} 1-2-1-14}$ & 3.4442 & 0.0009 \\
\hline $\mathrm{IM}_{\mathrm{R} 1-3-2-1}$ & 0.0000 & 0.5748 \\
\hline $\mathrm{IM}_{\mathrm{R} 1-3-2-2}$ & 0.5474 & 0.2279 \\
\hline $\mathrm{IM}_{\mathrm{R} 1-3-2-3}$ & 1.0673 & 0.0947 \\
\hline $\mathrm{IM}_{\mathrm{R} 1-3-2-4}$ & 1.8574 & 0.0249 \\
\hline $\mathrm{IM}_{\mathrm{R} 1-3-2-5}$ & 1.8926 & 0.0235 \\
\hline $\mathrm{IM}_{\mathrm{R} 1-3-2-6}$ & 2.1945 & 0.0141 \\
\hline $\mathrm{IM}_{\mathrm{R} 1-3-2-7}$ & 2.2541 & 0.0127 \\
\hline $\mathrm{IM}_{\mathrm{R} 1-3-2-8}$ & 2.8159 & 0.0049 \\
\hline $\mathrm{IM}_{\mathrm{R} 1-3-2-9}$ & 2.8938 & 0.0043 \\
\hline $\mathrm{IM}_{\mathrm{R} 1-3-2-10}$ & 2.9527 & 0.0039 \\
\hline $\mathrm{IM}_{\mathrm{R} 1-3-2-11}$ & 3.1622 & 0.0027 \\
\hline $\mathrm{IM}_{\mathrm{R} 1-3-2-12}$ & 3.1818 & 0.0027 \\
\hline $\mathrm{IM}_{\mathrm{R} 1-3-2-13}$ & 3.4937 & 0.0016 \\
\hline $\mathrm{IM}_{\mathrm{R} 1-3-2-14}$ & 3.5361 & 0.0015 \\
\hline $\mathrm{IM}_{\mathrm{R} 1-3-2-15}$ & 3.6709 & 0.0012 \\
\hline $\mathrm{IM}_{\mathrm{R} 1-3-2-16}$ & 3.6829 & 0.0011 \\
\hline $\mathrm{IM}_{\mathrm{R} 1-3-2-17}$ & 3.7930 & 0.0009 \\
\hline $\mathrm{IM}_{\mathrm{R} 1-3-2-18}$ & 3.8149 & 0.0009 \\
\hline $\mathrm{IM}_{\mathrm{R} 1-3-2-19}$ & 4.0322 & 0.0006 \\
\hline $\mathrm{IM}_{\mathrm{R} 1-3-2-20}$ & 4.2639 & 0.0004 \\
\hline
\end{tabular}




\begin{tabular}{|c|c|c|}
\hline $\mathrm{IM}_{\mathrm{R} 1-3-2-21}$ & 4.3948 & 0.0003 \\
\hline $\mathrm{IM}_{\mathrm{R} 1-3-2-22}$ & 4.7193 & 0.0002 \\
\hline $\mathrm{R}_{2}-1$ & 0.0000 & 0.2120 \\
\hline $\mathrm{R}_{2}-2$ & 0.1156 & 0.1744 \\
\hline $\mathrm{R}_{2}-3$ & 0.2673 & 0.1349 \\
\hline $\mathrm{R}_{2}-4$ & 0.3112 & 0.1253 \\
\hline $\mathrm{R}_{2-5}$ & 0.3812 & 0.1113 \\
\hline $\mathrm{R}_{2}-6$ & 0.5390 & 0.0853 \\
\hline $\mathrm{R}_{2-7}$ & 0.8485 & 0.0505 \\
\hline $\mathrm{R}_{2-8}$ & 0.9796 & 0.0405 \\
\hline $\mathrm{R}_{2}-9$ & 1.4317 & 0.0189 \\
\hline $\mathrm{R}_{2}-10$ & 2.0139 & 0.0071 \\
\hline $\mathrm{R}_{2}-11$ & 2.0292 & 0.0069 \\
\hline $\mathrm{R}_{2}-12$ & 2.1119 & 0.0060 \\
\hline $\mathrm{R}_{2}-13$ & 2.1282 & 0.0058 \\
\hline $\mathrm{R}_{2}-14$ & 2.2020 & 0.0051 \\
\hline $\mathrm{R}_{2}-15$ & 2.3476 & 0.0040 \\
\hline $\mathrm{R}_{2}-16$ & 2.4265 & 0.0035 \\
\hline $\mathrm{R}_{2-17}$ & 2.5024 & 0.0031 \\
\hline $\mathrm{R}_{2}-18$ & 2.8217 & 0.0018 \\
\hline $\mathrm{R}_{2}-19$ & 2.8845 & 0.0016 \\
\hline $\mathrm{R}_{2}-20$ & 3.0971 & 0.0011 \\
\hline $\mathrm{R}_{2}-21$ & 3.4655 & 0.0006 \\
\hline $\mathrm{R}_{2}-22$ & 4.0139 & 0.0002 \\
\hline $\mathrm{R}_{2}-23$ & 4.8621 & 0.0001 \\
\hline $\mathrm{IM}_{\mathrm{R} 2-1-1-1}$ & 0.0000 & 0.5499 \\
\hline $\mathrm{IM}_{\mathrm{R} 2-1-1-2}$ & 0.9130 & 0.1176 \\
\hline $\mathrm{IM}_{\mathrm{R} 2-1-1-3}$ & 1.2973 & 0.0614 \\
\hline $\mathrm{IM}_{\mathrm{R} 2-1-1-4}$ & 1.3489 & 0.0563 \\
\hline $\mathrm{IM}_{\mathrm{R} 2-1-1-5}$ & 1.3670 & 0.0546 \\
\hline $\mathrm{IM}_{\mathrm{R} 2-1-1-6}$ & 1.6090 & 0.0363 \\
\hline $\mathrm{IM}_{\mathrm{R} 2-1-1-7}$ & 1.6400 & 0.0344 \\
\hline $\mathrm{IM}_{\mathrm{R} 2-1-1-8}$ & 1.7773 & 0.0273 \\
\hline $\mathrm{IM}_{\mathrm{R} 2-1-1-9}$ & 1.9332 & 0.0210 \\
\hline $\mathrm{IM}_{\mathrm{R} 2-1-1-10}$ & 2.2408 & 0.0125 \\
\hline $\mathrm{IM}_{\mathrm{R} 2-1-1-11}$ & 2.4475 & 0.0088 \\
\hline $\mathrm{IM}_{\mathrm{R} 2-1-1-12}$ & 2.5027 & 0.0080 \\
\hline $\mathrm{IM}_{\mathrm{R} 2-1-1-13}$ & 2.8347 & 0.0046 \\
\hline
\end{tabular}




\begin{tabular}{|c|c|c|}
\hline $\mathrm{IM}_{\mathrm{R} 2-1-1-14}$ & 3.4815 & 0.0015 \\
\hline $\mathrm{IM}_{\mathrm{R} 2-1-1-15}$ & 3.5965 & 0.0013 \\
\hline $\mathrm{IM}_{\mathrm{R} 2-1-1-16}$ & 3.6227 & 0.0012 \\
\hline $\mathrm{IM}_{\mathrm{R} 2-1-1-17}$ & 3.6583 & 0.0011 \\
\hline $\mathrm{IM}_{\mathrm{R} 2-1-1-18}$ & 3.8248 & 0.0009 \\
\hline $\mathrm{IM}_{\mathrm{R} 2-1-1-19}$ & 4.0841 & 0.0006 \\
\hline $\mathrm{IM}_{\mathrm{R} 2-1-1-20}$ & 4.2095 & 0.0004 \\
\hline $\mathrm{IM}_{\mathrm{R} 2-1-1-21}$ & 4.7556 & 0.0002 \\
\hline $\mathrm{IM}_{\mathrm{R} 2-1-1-22}$ & 4.9809 & 0.0001 \\
\hline $\mathrm{IM}_{\mathrm{R} 2-1-2-1}$ & 0.0000 & 0.5838 \\
\hline $\mathrm{IM}_{\mathrm{R} 2-1-2-2}$ & 1.0146 & 0.1051 \\
\hline $\mathrm{IM}_{\mathrm{R} 2-1-2-3}$ & 1.1459 & 0.0842 \\
\hline $\mathrm{IM}_{\mathrm{R} 2-1-2-4}$ & 1.2526 & 0.0703 \\
\hline $\mathrm{IM}_{\mathrm{R} 2-1-2-5}$ & 1.3013 & 0.0648 \\
\hline $\mathrm{IM}_{\mathrm{R} 2-1-2-6}$ & 1.7122 & 0.0323 \\
\hline $\mathrm{IM}_{\mathrm{R} 2-1-2-7}$ & 1.9971 & 0.0200 \\
\hline $\mathrm{IM}_{\mathrm{R} 2-1-2-8}$ & 2.2261 & 0.0136 \\
\hline $\mathrm{IM}_{\mathrm{R} 2-1-2-9}$ & 2.4448 & 0.0094 \\
\hline $\mathrm{IM}_{\mathrm{R} 2-1-2-10}$ & 2.6795 & 0.0063 \\
\hline $\mathrm{IM}_{\mathrm{R} 2-1-2-11}$ & 3.1247 & 0.0030 \\
\hline $\mathrm{IM}_{\mathrm{R} 2-1-2-12}$ & 3.4367 & 0.0018 \\
\hline $\mathrm{IM}_{\mathrm{R} 2-1-2-13}$ & 3.5921 & 0.0013 \\
\hline $\mathrm{IM}_{\mathrm{R} 2-1-2-14}$ & 3.6135 & 0.0013 \\
\hline $\mathrm{IM}_{\mathrm{R} 2-1-2-15}$ & 3.7853 & 0.0010 \\
\hline $\mathrm{IM}_{\mathrm{R} 2-1-2-16}$ & 4.0402 & 0.0006 \\
\hline $\mathrm{IM}_{\mathrm{R} 2-1-2-17}$ & 4.2956 & 0.0004 \\
\hline $\mathrm{IM}_{\mathrm{R} 2-1-2-18}$ & 4.3169 & 0.0004 \\
\hline $\mathrm{IM}_{\mathrm{R} 2-1-2-19}$ & 4.6434 & 0.0002 \\
\hline $\mathrm{IM}_{\mathrm{R} 2-1-2-20}$ & 4.7662 & 0.0002 \\
\hline $\mathrm{IM}_{\mathrm{R} 2-2-1-1}$ & 0.0000 & 0.6753 \\
\hline $\mathrm{IM}_{\mathrm{R} 2-2-1-2}$ & 0.9319 & 0.1399 \\
\hline $\mathrm{IM}_{\mathrm{R} 2-2-1-3}$ & 1.7919 & 0.0327 \\
\hline $\mathrm{IM}_{\mathrm{R} 2-2-1-4}$ & 1.8254 & 0.0309 \\
\hline $\mathrm{IM}_{\mathrm{R} 2-2-1-5}$ & 1.8629 & 0.0290 \\
\hline $\mathrm{IM}_{\mathrm{R} 2-2-1}-6$ & 1.9009 & 0.0272 \\
\hline $\mathrm{IM}_{\mathrm{R} 2-2-1-7}$ & 2.3096 & 0.0136 \\
\hline $\mathrm{IM}_{\mathrm{R} 2-2-1-8}$ & 2.4792 & 0.0102 \\
\hline $\mathrm{IM}_{\mathrm{R} 2-2-1-9}$ & 2.4865 & 0.0101 \\
\hline
\end{tabular}




\begin{tabular}{|c|c|c|}
\hline $\mathrm{IM}_{\mathrm{R} 2-2-1-10}$ & 2.4942 & 0.0100 \\
\hline $\mathrm{IM}_{\mathrm{R} 2-2-1-11}$ & 2.5998 & 0.0084 \\
\hline $\mathrm{IM}_{\mathrm{R} 2-2-1-12}$ & 2.8626 & 0.0054 \\
\hline $\mathrm{IM}_{\mathrm{R} 2-2-1-13}$ & 3.6017 & 0.0015 \\
\hline $\mathrm{IM}_{\mathrm{R} 2-2-1-14}$ & 3.6321 & 0.0015 \\
\hline $\mathrm{IM}_{\mathrm{R} 2-2-1-15}$ & 3.6450 & 0.0014 \\
\hline $\mathrm{IM}_{\mathrm{R} 2-2-1-16}$ & 3.7270 & 0.0012 \\
\hline $\mathrm{IM}_{\mathrm{R} 2-2-1-17}$ & 3.8239 & 0.0011 \\
\hline $\mathrm{IM}_{\mathrm{R} 2-2-1-18}$ & 4.1748 & 0.0006 \\
\hline $\mathrm{IM}_{\mathrm{R} 2-3-1-1}$ & 0.0000 & 0.4454 \\
\hline $\mathrm{IM}_{\mathrm{R} 2-3-1-2}$ & 0.6970 & 0.1372 \\
\hline $\mathrm{IM}_{\mathrm{R} 2-3-1-3}$ & 0.7604 & 0.1232 \\
\hline $\mathrm{IM}_{\mathrm{R} 2-3-1-1}$ & 0.8293 & 0.1097 \\
\hline $\mathrm{IM}_{\mathrm{R} 2-3-1-5}$ & 1.1535 & 0.0634 \\
\hline $\mathrm{IM}_{\mathrm{R} 2-3-1-6}$ & 1.5358 & 0.0333 \\
\hline $\mathrm{IM}_{\mathrm{R} 2-3-1-7}$ & 1.5377 & 0.0331 \\
\hline $\mathrm{IM}_{\mathrm{R} 2-3-1-1-8}$ & 1.6866 & 0.0258 \\
\hline $\mathrm{IM}_{\mathrm{R} 2-3-1-9}$ & 2.3072 & 0.0090 \\
\hline $\mathrm{IM}_{\mathrm{R} 2-3-1-10}$ & 2.6329 & 0.0052 \\
\hline $\mathrm{IM}_{\mathrm{R} 2-3-1-11}$ & 2.9175 & 0.0032 \\
\hline $\mathrm{IM}_{\mathrm{R} 2-3-1-12}$ & 3.0036 & 0.0028 \\
\hline $\mathrm{IM}_{\mathrm{R} 2-3-1-13}$ & 3.2085 & 0.0020 \\
\hline $\mathrm{IM}_{\mathrm{R} 2-3-1-14}$ & 3.3619 & 0.0015 \\
\hline $\mathrm{IM}_{\mathrm{R} 2-3-1-15}$ & 3.4296 & 0.0014 \\
\hline $\mathrm{IM}_{\mathrm{R} 2-3-1-16}$ & 3.4998 & 0.0012 \\
\hline $\mathrm{IM}_{\mathrm{R} 2-3-1-17}$ & 3.6910 & 0.0009 \\
\hline $\mathrm{IM}_{\mathrm{R} 2-3-1-18}$ & 3.7572 & 0.0008 \\
\hline $\mathrm{IM}_{\mathrm{R} 2-3-1-19}$ & 4.3769 & 0.0003 \\
\hline $\mathrm{IM}_{\mathrm{R} 2-3-1-20}$ & 4.5545 & 0.0002 \\
\hline $\mathrm{IM}_{\mathrm{R} 2-3-1-21}$ & 4.6936 & 0.0002 \\
\hline $\mathrm{IM}_{\mathrm{R} 2-3-1-22}$ & 4.8454 & 0.0001 \\
\hline $\mathrm{IM}_{\mathrm{R} 2-3-1-23}$ & 4.9202 & 0.0001 \\
\hline $\mathrm{IM}_{\mathrm{R} 2-3-2-1}$ & 0.0000 & 0.6321 \\
\hline IM $\mathrm{M}_{\mathrm{R}-3-3-2-2}$ & 0.8450 & 0.1516 \\
\hline $\mathrm{IM}_{\mathrm{R} 2-3-2-3}$ & 1.2492 & 0.0766 \\
\hline $\mathrm{IM}_{\mathrm{R} 2-3-2-4}$ & 1.3903 & 0.0603 \\
\hline $\mathrm{IM}_{\mathrm{R} 2-3-2-5}$ & 1.9101 & 0.0251 \\
\hline $\mathrm{IM}_{\mathrm{R} 2-3-2-6}$ & 2.0188 & 0.0209 \\
\hline
\end{tabular}




\begin{tabular}{|c|c|c|}
\hline $\mathrm{IM}_{\mathrm{R} 2-3-2-7}$ & 2.5569 & 0.0084 \\
\hline $\mathrm{IM}_{\mathrm{R} 2-3-2-8}$ & 2.8851 & 0.0048 \\
\hline $\mathrm{IM}_{\mathrm{R} 2-3-2-9}$ & 2.9603 & 0.0043 \\
\hline $\mathrm{IM}_{\mathrm{R} 2-3-2-10}$ & 3.0927 & 0.0034 \\
\hline $\mathrm{IM}_{\mathrm{R} 2-3-2-11}$ & 3.2490 & 0.0026 \\
\hline $\mathrm{IM}_{\mathrm{R} 2-3-2-12}$ & 3.3333 & 0.0023 \\
\hline $\mathrm{IM}_{\mathrm{R} 2-3-2-13}$ & 3.4978 & 0.0017 \\
\hline $\mathrm{IM}_{\mathrm{R} 2-3-2-14}$ & 3.6650 & 0.0013 \\
\hline $\mathrm{IM}_{\mathrm{R2} 2-3-2-15}$ & 3.7723 & 0.0011 \\
\hline $\mathrm{IM}_{\mathrm{R} 2-3-2-16}$ & 3.8424 & 0.0010 \\
\hline $\mathrm{IM}_{\mathrm{R} 2-3-2-17}$ & 3.8609 & $0.000 s$ \\
\hline $\mathrm{IM}_{\mathrm{R} 2-3-2-18}$ & 3.9073 & 0.0005 \\
\hline $\mathrm{IM}_{\mathrm{R} 2-3-2-19}$ & 4.2642 & 0.0005 \\
\hline $\mathrm{IM}_{\mathrm{R} 2-3-2-20}$ & 4.5190 & 0.0003 \\
\hline $\mathrm{IM}_{\mathrm{R} 2-3-2-21}$ & 4.9351 & 0.0002 \\
\hline $\mathrm{IM}_{\mathrm{R} 2-4-1-1}$ & 0.0000 & 0.3514 \\
\hline $\mathrm{IM}_{\mathrm{R} 2-4-1-2}$ & 0.3279 & 0.2019 \\
\hline $\mathrm{IM}_{\mathrm{R} 2-4-1-3}$ & 0.5941 & 0.1288 \\
\hline $\mathrm{IM}_{\mathrm{R} 2-4-1-4}$ & 0.7385 & $0.100 s$ \\
\hline $\mathrm{IM}_{\mathrm{R} 2-4-1-5}$ & 1.0545 & 0.0592 \\
\hline $\mathrm{IM}_{\mathrm{R} 2-4-1-6}$ & 1.1315 & 0.0519 \\
\hline $\mathrm{IM}_{\mathrm{R} 2-4-1-7}$ & 1.2423 & 0.0431 \\
\hline $\mathrm{IM}_{\mathrm{R} 2-4-1-8}$ & 1.5592 & 0.0252 \\
\hline $\mathrm{IM}_{\mathrm{R} 2-4-1-9}$ & 2.0312 & 0.0114 \\
\hline $\mathrm{IM}_{\mathrm{R} 2-4-1-10}$ & 2.0821 & 0.0104 \\
\hline $\mathrm{IM}_{\mathrm{R} 2-4-1-11}$ & 2.5379 & 0.0048 \\
\hline $\mathrm{IM}_{\mathrm{R} 2-4-1-12}$ & 2.9363 & 0.0025 \\
\hline $\mathrm{IM}_{\mathrm{R} 2-4-1-13}$ & 2.9814 & 0.0023 \\
\hline $\mathrm{IM}_{\mathrm{R} 2-4-1-14}$ & 3.3214 & 0.0013 \\
\hline $\mathrm{IM}_{\mathrm{R} 2-4-1-15}$ & 3.3395 & 0.0012 \\
\hline $\mathrm{IM}_{\mathrm{R} 2-4-1-16}$ & 3.4129 & 0.0011 \\
\hline $\mathrm{IM}_{\mathrm{R} 2-4-1-17}$ & 3.5234 & $0.000 \mathrm{~S}$ \\
\hline $\mathrm{IM}_{\mathrm{R} 2-4-1-18}$ & 3.5628 & $0.000 s$ \\
\hline $\mathrm{IM}_{\mathrm{R} 2-4-1-19}$ & 3.8192 & 0.0006 \\
\hline $\mathrm{IM}_{\mathrm{R} 2-4-1-20}$ & 3.9086 & 0.0005 \\
\hline $\mathrm{IM}_{\mathrm{R} 2-5-1-1}$ & 0.0000 & 0.3678 \\
\hline $\mathrm{IM}_{\mathrm{R} 2-5-1-2}$ & 0.4339 & 0.1767 \\
\hline $\mathrm{IM}_{\mathrm{R} 2-5-1-3}$ & 0.4391 & 0.1752 \\
\hline
\end{tabular}




\begin{tabular}{|c|c|c|}
\hline $\mathrm{IM}_{\mathrm{R} 2-5-1-4}$ & 0.6455 & 0.1236 \\
\hline $\mathrm{IM}_{\mathrm{R} 2-5-1-5}$ & 1.0519 & 0.0622 \\
\hline $\mathrm{IM}_{\mathrm{R} 2-5-1-6}$ & 1.1484 & 0.0528 \\
\hline $\mathrm{IM}_{\mathrm{R} 2-5-1-7}$ & 2.3196 & 0.0073 \\
\hline $\mathrm{IM}_{\mathrm{R} 2-5-1-8}$ & 2.4795 & 0.0056 \\
\hline $\mathrm{IM}_{\mathrm{R} 2-5-1-9}$ & 2.5409 & 0.0050 \\
\hline $\mathrm{IM}_{\mathrm{R} 2-5-1-10}$ & 2.5507 & 0.0049 \\
\hline $\mathrm{IM}_{\mathrm{R} 2-5-1-11}$ & 2.7501 & 0.0035 \\
\hline $\mathrm{IM}_{\mathrm{R} 2-5-1-12}$ & 2.7538 & 0.0035 \\
\hline $\mathrm{IM}_{\mathrm{R} 2-5-1-13}$ & 2.8049 & 0.0032 \\
\hline $\mathrm{IM}_{\mathrm{R} 2-5-1-14}$ & 3.0497 & 0.0021 \\
\hline $\mathrm{IM}_{\mathrm{R} 2-5-1-15}$ & 3.1281 & 0.0019 \\
\hline $\mathrm{IM}_{\mathrm{R} 2-5-1-16}$ & 3.2632 & 0.0015 \\
\hline $\mathrm{IM}_{\mathrm{R} 2-5-1-17}$ & 3.3177 & 0.0014 \\
\hline $\mathrm{IM}_{\mathrm{R} 2-5-1-18}$ & 3.5695 & 0.0009 \\
\hline $\mathrm{IM}_{\mathrm{R} 2-5-1-19}$ & 3.7905 & 0.0006 \\
\hline $\mathrm{IM}_{\mathrm{R} 2-5-1-20}$ & 4.5936 & 0.0002 \\
\hline $\mathrm{IM}_{\mathrm{R} 2-5-1-21}$ & 4.8953 & 0.0001 \\
\hline $\mathrm{IM}_{\mathrm{R} 2-5-2-1}$ & 0.0000 & 0.3769 \\
\hline $\mathrm{IM}_{\mathrm{R} 2-5-2-2}$ & 0.2052 & 0.2665 \\
\hline $\mathrm{IM}_{\mathrm{R} 2-5-2-3}$ & 0.4420 & 0.1786 \\
\hline $\mathrm{IM}_{\mathrm{R} 2-5-2-4}$ & 1.0130 & 0.0681 \\
\hline $\mathrm{IM}_{\mathrm{R} 2-5-2-5}$ & 1.6746 & 0.0223 \\
\hline $\mathrm{IM}_{\mathrm{R} 2-5-2-6}$ & 1.7836 & 0.0185 \\
\hline $\mathrm{IM}_{\mathrm{R} 2-5-2-7}$ & 1.8448 & 0.0167 \\
\hline $\mathrm{IM}_{\mathrm{R} 2-5-2-8}$ & 2.0812 & 0.0112 \\
\hline $\mathrm{IM}_{\mathrm{R} 2-5-2-9}$ & 2.1368 & 0.0102 \\
\hline $\mathrm{IM}_{\mathrm{R} 2-5-2-10}$ & 2.1970 & 0.0092 \\
\hline $\mathrm{IM}_{\mathrm{R} 2-5-2-11}$ & 2.2374 & 0.0086 \\
\hline $\mathrm{IM}_{\mathrm{R} 2-5-2-12}$ & 2.9286 & 0.0027 \\
\hline $\mathrm{IM}_{\mathrm{R} 2-5-2-13}$ & 2.9394 & 0.0026 \\
\hline $\mathrm{IM}_{\mathrm{R} 2-5-2-14}$ & 3.0194 & 0.0023 \\
\hline $\mathrm{IM}_{\mathrm{R} 2-5-2-15}$ & 3.0367 & 0.0022 \\
\hline $\mathrm{IM}_{\mathrm{R} 2-5-2-16}$ & 3.2460 & 0.0016 \\
\hline $\mathrm{IM}_{\mathrm{R} 2-5-2-17}$ & 3.7144 & 0.0007 \\
\hline $\mathrm{IM}_{\mathrm{R} 2-5-2-18}$ & 3.8559 & 0.0006 \\
\hline $\mathrm{IM}_{\mathrm{R} 2-5-2-19}$ & 3.9785 & 0.0005 \\
\hline $\mathrm{IM}_{\mathrm{R} 2-5-2-20}$ & 4.4758 & 0.0002 \\
\hline
\end{tabular}




\begin{tabular}{|c|c|c|}
\hline $\mathrm{IM}_{\mathrm{R} 2-6-1-1}$ & 0.0000 & 0.6694 \\
\hline $\mathrm{IM}_{\mathrm{R} 2-6-1-2}$ & 1.3017 & 0.0742 \\
\hline $\mathrm{IM}_{\mathrm{R} 2-6-1-3}$ & 1.5021 & 0.0529 \\
\hline $\mathrm{IM}_{\mathrm{R} 2-6-1-4}$ & 1.6848 & 0.0388 \\
\hline IM ${ }_{\text {R2-6-1- }-5}$ & 1.7563 & 0.0344 \\
\hline $\mathrm{IM}_{\mathrm{R} 2-6-1-6}$ & 1.7784 & 0.0332 \\
\hline $\mathrm{IM}_{\mathrm{R} 2-6-1-7}$ & 1.8103 & 0.0314 \\
\hline $\mathrm{IM}_{\mathrm{R} 2-6-1-8}$ & 1.8554 & 0.0291 \\
\hline $\mathrm{IM}_{\mathrm{R} 2-6-1-9}$ & 2.2959 & 0.0138 \\
\hline $\mathrm{IM}_{\mathrm{R} 2-6-1-10}$ & 2.5922 & 0.0084 \\
\hline $\mathrm{IM}_{\mathrm{R} 2-6-1-11}$ & 2.9931 & 0.0043 \\
\hline $\mathrm{IM}_{\mathrm{R} 2-6-1}-12$ & 3.2621 & 0.0027 \\
\hline $\mathrm{IM}_{\mathrm{R} 2-6-1-13}$ & 3.2732 & 0.0027 \\
\hline $\mathrm{IM}_{\mathrm{R} 2-6-1}-14$ & 3.5976 & 0.0015 \\
\hline $\mathrm{IM}_{\mathrm{R} 2-6-1-15}$ & 3.6991 & 0.0013 \\
\hline $\mathrm{IM}_{\mathrm{R} 2-6-1-16}$ & 4.0126 & 0.0008 \\
\hline $\mathrm{IM}_{\mathrm{R} 2-6-1-17}$ & 4.0245 & 0.0007 \\
\hline $\mathrm{IM}_{\mathrm{R} 2-6-1-18}$ & 4.8790 & 0.0002 \\
\hline $\mathrm{IM}_{\mathrm{R} 2-6-1-19}$ & 4.9780 & 0.0001 \\
\hline $\mathrm{IM}_{\mathrm{R} 2-7-1-1}$ & 0.0000 & 0.2717 \\
\hline $\mathrm{IM}_{\mathrm{R} 2-7-1-2}$ & 0.0043 & 0.2697 \\
\hline $\mathrm{IM}_{\mathrm{R} 2-7-1-3}$ & 0.1974 & 0.1946 \\
\hline $\mathrm{IM}_{\mathrm{R} 2-7-1-4}$ & 0.2304 & 0.1841 \\
\hline $\mathrm{IM}_{\mathrm{R} 2-7-1-5}$ & 0.9485 & 0.0547 \\
\hline $\mathrm{IM}_{\mathrm{R} 2-7-1-6}$ & 1.8709 & 0.0115 \\
\hline $\mathrm{IM}_{\mathrm{R} 2-7-1-7}$ & 2.4635 & 0.0042 \\
\hline $\mathrm{IM}_{\mathrm{R} 2-7-1-8}$ & 2.5169 & 0.0039 \\
\hline $\mathrm{IM}_{\mathrm{R} 2-7-1-9}$ & 2.9818 & 0.0018 \\
\hline $\mathrm{IM}_{\mathrm{R} 2-7-1-10}$ & 3.2027 & 0.0012 \\
\hline $\mathrm{IM}_{\mathrm{R} 2-7-1-11}$ & 3.3724 & 0.0009 \\
\hline $\mathrm{IM}_{\mathrm{R} 2-7-1-12}$ & 3.6141 & 0.0006 \\
\hline $\mathrm{IM}_{\mathrm{R} 2-7-1-13}$ & 3.8983 & 0.0004 \\
\hline $\mathrm{IM}_{\mathrm{R} 2-7-1-14}$ & 4.1046 & 0.0003 \\
\hline $\mathrm{IM}_{\mathrm{R} 2-7-1-15}$ & 4.3058 & 0.0002 \\
\hline $\mathrm{IM}_{\mathrm{R} 2-7-1-16}$ & 4.5222 & 0.0001 \\
\hline $\mathrm{IM}_{\mathrm{R} 2-7-1-17}$ & 4.6375 & 0.0001 \\
\hline $\mathrm{IM}_{\mathrm{R} 2-7-1}-18$ & 4.7973 & 0.0001 \\
\hline \multirow[t]{2}{*}{$\mathrm{IM}_{\mathrm{R} 2-7-2-1}$} & 0.0000 & 0.1973 \\
\hline & $\mathrm{S} 23$ & \\
\hline
\end{tabular}




\begin{tabular}{|c|c|c|}
\hline $\mathrm{IM}_{\mathrm{R} 2-7-2-2}$ & 0.0243 & 0.1894 \\
\hline $\mathrm{IM}_{\mathrm{R} 2-7-2-3}$ & 0.0388 & 0.1848 \\
\hline $\mathrm{IM}_{\mathrm{R} 2-7-2-4}$ & 0.1557 & 0.1517 \\
\hline $\mathrm{IM}_{\mathrm{R} 2-7-2-5}$ & 0.5340 & 0.0800 \\
\hline $\mathrm{IM}_{\mathrm{R} 2-7-2-6}$ & 0.6682 & 0.0638 \\
\hline $\mathrm{IM}_{\mathrm{R} 2-7-2-7}$ & 0.8720 & 0.0452 \\
\hline $\mathrm{IM}_{\mathrm{R} 2-7-2-8}$ & 1.2619 & 0.0234 \\
\hline $\mathrm{IM}_{\mathrm{R} 2-7-2-9}$ & 1.3955 & 0.0187 \\
\hline $\mathrm{IM}_{\mathrm{R} 2-7-2-10}$ & 1.4054 & 0.0184 \\
\hline $\mathrm{IM}_{\mathrm{R} 2-7-2-11}$ & 1.7149 & 0.0109 \\
\hline $\mathrm{IM}_{\mathrm{R} 2-7-2-12}$ & 2.2260 & 0.0046 \\
\hline $\mathrm{IM}_{\mathrm{R} 2-7-2-13}$ & 2.3425 & 0.0038 \\
\hline $\mathrm{IM}_{\mathrm{R} 2-7-2-14}$ & 2.7849 & 0.0018 \\
\hline $\mathrm{IM}_{\mathrm{R} 2-7-2-15}$ & 2.8819 & 0.0015 \\
\hline $\mathrm{IM}_{\mathrm{R} 2-7-2-16}$ & 2.9245 & 0.0014 \\
\hline $\mathrm{IM}_{\mathrm{R} 2-7-2-17}$ & 3.2127 & 0.0009 \\
\hline $\mathrm{IM}_{\mathrm{R} 2-7-2-18}$ & 3.2556 & 0.0008 \\
\hline $\mathrm{IM}_{\mathrm{R} 2-7-2-19}$ & 3.2870 & 0.0008 \\
\hline $\mathrm{IM}_{\mathrm{R} 2-7-2-20}$ & 3.3846 & 0.0006 \\
\hline $\mathrm{IM}_{\mathrm{R} 2-7-2-21}$ & 4.2676 & 0.0001 \\
\hline $\mathrm{IM}_{\mathrm{R} 2-7-2-22}$ & 4.6205 & 0.0001 \\
\hline $\mathrm{IM}_{\mathrm{R} 2-8-1-1}$ & 0.0000 & 0.5401 \\
\hline $\mathrm{IM}_{\mathrm{R} 2-8-1-2}$ & 0.5205 & 0.2241 \\
\hline $\mathrm{IM}_{\mathrm{R} 2-8-1-3}$ & 0.8546 & 0.1275 \\
\hline $\mathrm{IM}_{\mathrm{R} 2-8-1-4}$ & 1.6620 & 0.0326 \\
\hline $\mathrm{IM}_{\mathrm{R} 2-8-1-5}$ & 1.9338 & 0.0206 \\
\hline $\mathrm{IM}_{\mathrm{R} 2-8-1}-6$ & 1.9775 & 0.0191 \\
\hline $\mathrm{IM}_{\mathrm{R} 2-8-1-7}$ & 2.4317 & 0.0089 \\
\hline $\mathrm{IM}_{\mathrm{R} 2-8-1-8}$ & 2.4777 & 0.0082 \\
\hline $\mathrm{IM}_{\mathrm{R} 2-8-1-9}$ & 2.8798 & 0.0042 \\
\hline $\mathrm{IM}_{\mathrm{R} 2-8-1-10}$ & 2.9044 & 0.0040 \\
\hline $\mathrm{IM}_{\mathrm{R} 2-8-1-11}$ & 3.1290 & 0.0027 \\
\hline $\mathrm{IM}_{\mathrm{R} 2-8-1-12}$ & 3.1849 & 0.0025 \\
\hline $\mathrm{IM}_{\mathrm{R} 2-8-1-13}$ & 3.3304 & 0.0019 \\
\hline $\mathrm{IM}_{\mathrm{R} 2-8-1-14}$ & 3.4206 & 0.0017 \\
\hline $\mathrm{IM}_{\mathrm{R} 2-8-1-15}$ & 3.7146 & 0.0010 \\
\hline $\mathrm{IM}_{\mathrm{R} 2-8-1-16}$ & 3.8272 & 0.0008 \\
\hline $\mathrm{IM}_{\mathrm{R} 2-8-1-17}$ & 4.9945 & 0.0001 \\
\hline
\end{tabular}




\begin{tabular}{lll}
\hline TMP-RO-1 & 0.0000 & 0.3823 \\
TMP-RO-2 & 0.4354 & 0.1832 \\
TMP-RO-3 & 0.5523 & 0.1504 \\
TMP-RO-4 & 0.8764 & 0.0870 \\
TMP-RO-5 & 0.9282 & 0.0797 \\
TMP-RO-6 & 1.0589 & 0.0639 \\
TMP-RO-7 & 1.6530 & 0.0234 \\
TMP-RO-8 & 1.6549 & 0.0233 \\
TMP-RO-9 & 2.6948 & 0.0040 \\
TMP-RO-10 & 3.0488 & 0.0022 \\
TMP-RO-11 & 3.8360 & 0.0006 \\
\hline TCPP-RO-1 & 0.0000 & 0.3171 \\
TCPP-RO-2 & 0.2048 & 0.2244 \\
TCPP-RO-3 & 0.2676 & 0.2018 \\
TCPP-RO-4 & 0.6528 & 0.1052 \\
TCPP-RO-5 & 1.0542 & 0.0534 \\
TCPP-RO-6 & 1.1527 & 0.0452 \\
TCPP-RO-7 & 1.5575 & 0.0228 \\
TCPP-RO-8 & 1.7017 & 0.0179 \\
TCPP-RO-9 & 2.3370 & 0.0061 \\
TCPP-RO-10 & 2.9014 & 0.0024 \\
TCPP-RO-11 & 3.0471 & 0.0018 \\
TCPP-RO-12 & 3.3058 & 0.0012 \\
TCPP-RO-13 & 3.8649 & 0.0005 \\
TCPP-RO-14 & 4.3405 & 0.0002 \\
\hline
\end{tabular}

197 
198 Table S3. Calculated multi-conformer reaction rate constants $\left(k_{\mathrm{MC}-\mathrm{TST}}\right)$, corresponding 199 available single-conformer reaction rate constant $\left(k_{\mathrm{SC}-\mathrm{TST} i}\right)$ of transition state $i\left(\mathrm{TS}_{i}\right)$ and 200 relative Boltzmann populations $\left(w_{i}\right)$ of the reactant conformer connected to $\mathrm{TS}_{i}$ for the 201 selected crucial reaction pathways based on the energetic data at the M06-2X/6$202311+\mathrm{G}(3 \mathrm{df}, 2 \mathrm{p}) / / \mathrm{M} 06-2 \mathrm{X} / 6-31+\mathrm{G}(\mathrm{d}, \mathrm{p})$ level of theory.

\begin{tabular}{|c|c|c|c|c|}
\hline Reaction pathways & TSs & $k_{\text {SC-TST } i}\left(\mathrm{~s}^{-1}\right)$ & $w_{i}$ & $k_{\text {MC-TST }}\left(\mathrm{s}^{-1}\right)$ \\
\hline \multirow{3}{*}{$\mathrm{R}_{1} \rightarrow \mathrm{IM}_{\mathrm{R} 1-1-1}$} & $\mathrm{TS}_{\mathrm{R} 1-1-1-1}$ & 0.43 & 0.1806 & 0.11 \\
\hline & $\mathrm{TS}_{\mathrm{R} 1-1-1-2}$ & $4.6 \times 10^{-3}$ & 0.1046 & \\
\hline & $\mathrm{TS}_{\mathrm{R} 1-1-1-3}$ & 1.4 & 0.0194 & \\
\hline \multirow[t]{3}{*}{$\mathrm{IM}_{\mathrm{R} 1-1-1} \rightarrow \mathrm{R}_{1}$} & $\mathrm{TS}_{\mathrm{R} 1-1-1-1}$ & $3.4 \times 10^{5}$ & 0.0375 & $1.6 \times 10^{4}$ \\
\hline & $\mathrm{TS}_{\mathrm{R} 1-1-1-2}$ & $2.1 \times 10^{5}$ & 0.0126 & \\
\hline & $\mathrm{TS}_{\mathrm{R} 1-1-1-3}$ & $2.9 \times 10^{5}$ & 0.0002 & \\
\hline \multirow[t]{3}{*}{$\mathrm{IM}_{\mathrm{R} 1-2-1} \rightarrow \mathrm{P}_{\mathrm{R} 1-3-1}$} & $\mathrm{TS}_{\mathrm{R} 1-3-1-1}$ & 3.8 & 0.3110 & 1.3 \\
\hline & $\mathrm{TS}_{\mathrm{R} 1-3-1-2}$ & 1.9 & 0.0383 & \\
\hline & $\mathrm{TS}_{\mathrm{R} 1-3-1-3}$ & 71.4 & 0.0009 & \\
\hline \multirow[t]{4}{*}{$\mathrm{IM}_{\mathrm{R} 1-2-1} \rightarrow \mathrm{P}_{\mathrm{R} 1-3-2}$} & $\mathrm{TS}_{\mathrm{R} 1-3-2-1}$ & 0.10 & 0.0144 & $5.8 \times 10^{-3}$ \\
\hline & $\mathrm{TS}_{\mathrm{R} 1-3-2-2}$ & 0.25 & 0.0026 & \\
\hline & $\mathrm{TS}_{\mathrm{R} 1-3-2-3}$ & 2.9 & 0.0012 & \\
\hline & $\mathrm{TS}_{\mathrm{R} 1-3-2-4}$ & 0.18 & 0.0009 & \\
\hline \multirow[t]{4}{*}{$\mathrm{P}_{\mathrm{R} 1-3-2} \rightarrow \mathrm{IM}_{\mathrm{R} 1-2-1}$} & $\mathrm{TS}_{\mathrm{R} 1-3-2-1}$ & $3.7 \times 10^{7}$ & 0.0003 & $6.4 \times 10^{4}$ \\
\hline & $\mathrm{TS}_{\mathrm{R} 1-3-2-2}$ & $2.5 \times 10^{6}$ & 0.0004 & \\
\hline & $\mathrm{TS}_{\mathrm{R} 1-3-2-3}$ & $2.4 \times 10^{8}$ & 0.0002 & \\
\hline & $\mathrm{TS}_{\mathrm{R} 1-3-2-4}$ & $1.8 \times 10^{6}$ & 0.0009 & \\
\hline \multirow[t]{4}{*}{$\mathrm{R}_{2} \rightarrow \mathrm{IM}_{\mathrm{R} 2-1-1}$} & $\mathrm{TS}_{\mathrm{R} 2-1-1-1}$ & 13.9 & 0.1349 & 7.3 \\
\hline & $\mathrm{TS}_{\mathrm{R} 2-1-1-2}$ & 86.4 & 0.0505 & \\
\hline & $\mathrm{TS}_{\mathrm{R} 2-1-1-3}$ & 19.4 & 0.0405 & \\
\hline & $\mathrm{TS}_{\mathrm{R} 2-1-1}-4$ & 73.2 & 0.0040 & \\
\hline \multirow[t]{4}{*}{$\mathrm{IM}_{\mathrm{R} 2-1-1} \rightarrow \mathrm{R}_{2}$} & $\mathrm{TS}_{\mathrm{R} 2-1-1-1}$ & $8.3 \times 10^{4}$ & 0.0125 & $4.3 \times 10^{3}$ \\
\hline & $\mathrm{TS}_{\mathrm{R} 2-1-1-2}$ & $7.5 \times 10^{5}$ & 0.0012 & \\
\hline & $\mathrm{TS}_{\mathrm{R} 2-1-1-3}$ & $8.8 \times 10^{5}$ & 0.0006 & \\
\hline & $\mathrm{TS}_{\mathrm{R} 2-1-1-4}$ & $1.2 \times 10^{6}$ & 0.0015 & \\
\hline $\mathrm{R}_{2} \rightarrow \mathrm{IM}_{\mathrm{R} 2-1-2}$ & $\mathrm{TS}_{\mathrm{R} 2-1-2-1}$ & 1.0 & 0.0060 & $5.8 \times 10^{-3}$ \\
\hline
\end{tabular}




\begin{tabular}{|c|c|c|c|c|}
\hline & $\mathrm{TS}_{\mathrm{R} 2-1-2-2}$ & 0.12 & 0.0002 & \\
\hline & $\mathrm{TS}_{\mathrm{R} 2-1-2-3}$ & 0.53 & 0.0001 & \\
\hline \multirow[t]{3}{*}{$\mathrm{IM}_{\mathrm{R} 2-1-2} \rightarrow \mathrm{R}_{2}$} & $\mathrm{TS}_{\mathrm{R} 2-1-2-1}$ & $2.1 \times 10^{7}$ & 0.0018 & $3.6 \times 10^{5}$ \\
\hline & $\mathrm{TS}_{\mathrm{R} 2-1-2-2}$ & $1.6 \times 10^{7}$ & 0.0010 & \\
\hline & $\mathrm{TS}_{\mathrm{R} 2-1-2-3}$ & $9.7 \times 10^{6}$ & 0.0323 & \\
\hline \multirow[t]{4}{*}{$\mathrm{IM}_{\mathrm{R} 2-2-1} \rightarrow \mathrm{IM}_{\mathrm{R} 2-3-1}$} & $\mathrm{TS}_{\mathrm{R} 2-3-1-1}$ & 19.1 & 0.1399 & 6.2 \\
\hline & $\mathrm{TS}_{\mathrm{R} 2-3-1-2}$ & 5.1 & 0.0327 & \\
\hline & $\mathrm{TS}_{\mathrm{R} 2-3-1-3}$ & $1.0 \times 10^{2}$ & 0.0290 & \\
\hline & $\mathrm{TS}_{\mathrm{R} 2-3-1-4}$ & $4.4 \times 10^{2}$ & 0.0011 & \\
\hline \multirow[t]{4}{*}{$\mathrm{IM}_{\mathrm{R} 2-3-1} \rightarrow \mathrm{IM}_{\mathrm{R} 2-2-1}$} & $\mathrm{TS}_{\mathrm{R} 2-3-1-1}$ & $1.5 \times 10^{5}$ & 0.0015 & $7.0 \times 10^{3}$ \\
\hline & $\mathrm{TS}_{\mathrm{R} 2-3-1-2}$ & $1.5 \times 10^{6}$ & 0.0001 & \\
\hline & $\mathrm{TS}_{\mathrm{R} 2-3-1-3}$ & $2.7 \times 10^{4}$ & 0.1372 & \\
\hline & $\mathrm{TS}_{\mathrm{R} 2-3-1-4}$ & $3.2 \times 10^{6}$ & 0.0009 & \\
\hline \multirow[t]{3}{*}{$\mathrm{IM}_{\mathrm{R} 2-2-1} \rightarrow \mathrm{IM}_{\mathrm{R} 2-3-2}$} & $\mathrm{TS}_{\mathrm{R} 2-3-2-1}$ & 0.60 & 0.0100 & $9.3 \times 10^{-3}$ \\
\hline & $\mathrm{TS}_{\mathrm{R} 2-3-2-2}$ & 1.5 & 0.0015 & \\
\hline & $\mathrm{TS}_{\mathrm{R} 2-3-2-3}$ & 1.7 & 0.0006 & \\
\hline \multirow[t]{3}{*}{$\mathrm{IM}_{\mathrm{R} 2-3-2} \rightarrow \mathrm{IM}_{\mathrm{R} 2-2-1}$} & $\mathrm{TS}_{\mathrm{R} 2-3-2-1}$ & $2.1 \times 10^{7}$ & 0.0043 & $1.3 \times 10^{5}$ \\
\hline & $\mathrm{TS}_{\mathrm{R} 2-3-2-2}$ & $1.3 \times 10^{7}$ & 0.0026 & \\
\hline & $\mathrm{TS}_{\mathrm{R} 2-3-2-3}$ & $1.8 \times 10^{7}$ & 0.0003 & \\
\hline \multirow[t]{4}{*}{$\mathrm{IM}_{\mathrm{R} 2-4-1} \rightarrow \mathrm{IM}_{\mathrm{R} 2-5-1}$} & $\mathrm{TS}_{\mathrm{R} 2-5-1-1}$ & 3.0 & 0.2019 & 0.65 \\
\hline & $\mathrm{TS}_{\mathrm{R} 2-5-1-2}$ & 1.4 & 0.0431 & \\
\hline & $\mathrm{TS}_{\mathrm{R} 2-5-1-3}$ & 3.2 & 0.0025 & \\
\hline & $\mathrm{TS}_{\mathrm{R} 2-5-1-4}$ & 28.1 & 0.0013 & \\
\hline \multirow[t]{4}{*}{$\mathrm{IM}_{\mathrm{R} 2-5-1} \rightarrow \mathrm{IM}_{\mathrm{R} 2-4-1}$} & $\mathrm{TS}_{\mathrm{R} 2-5-1-1}$ & $7.0 \times 10^{4}$ & 0.0528 & $4.6 \times 10^{3}$ \\
\hline & $\mathrm{TS}_{\mathrm{R} 2-5-1-2}$ & $4.0 \times 10^{4}$ & 0.0032 & \\
\hline & $\mathrm{TS}_{\mathrm{R} 2-5-1-3}$ & $1.5 \times 10^{4}$ & 0.0056 & \\
\hline & $\mathrm{TS}_{\mathrm{R} 2-5-1-4}$ & $8.3 \times 10^{5}$ & 0.0009 & \\
\hline \multirow[t]{3}{*}{$\mathrm{IM}_{\mathrm{R} 2-4-1} \rightarrow \mathrm{IM}_{\mathrm{R} 2-5-2}$} & $\mathrm{TS}_{\mathrm{R} 2-5-2-1}$ & $7.6 \times 10^{-4}$ & 0.3537 & $7.8 \times 10^{-4}$ \\
\hline & $\mathrm{TS}_{\mathrm{R} 2-5-2-2}$ & 0.39 & 0.0012 & \\
\hline & $\mathrm{TS}_{\mathrm{R} 2-5-2-3}$ & 0.03 & 0.0005 & \\
\hline \multirow[t]{3}{*}{$\mathrm{IM}_{\mathrm{R} 2-5-2} \rightarrow \mathrm{IM}_{\mathrm{R} 2-4-1}$} & $\mathrm{TS}_{\mathrm{R} 2-5-2-1}$ & $1.3 \times 10^{8}$ & 0.0006 & $1.7 \times 10^{5}$ \\
\hline & $\mathrm{TS}_{\mathrm{R} 2-5-2-2}$ & $4.9 \times 10^{3}$ & 0.0026 & \\
\hline & $\mathrm{TS}_{\mathrm{R} 2-5-2-3}$ & $6.4 \times 10^{7}$ & 0.0016 & \\
\hline \multirow[t]{5}{*}{$\mathrm{IM}_{\mathrm{R} 2-6-1} \rightarrow \mathrm{IM}_{\mathrm{R} 2-7-1}$} & $\mathrm{TS}_{\mathrm{R} 2-7-1-1}$ & 1.4 & 0.0388 & 0.08 \\
\hline & $\mathrm{TS}_{\mathrm{R} 2-7-1-2}$ & 0.70 & 0.0138 & \\
\hline & $\mathrm{TS}_{\mathrm{R} 2-7-1-3}$ & 4.1 & 0.0027 & \\
\hline & $\mathrm{TS}_{\mathrm{R} 2-7-1-4}$ & 0.03 & 0.0008 & \\
\hline & $\mathrm{TS}_{\mathrm{R} 2-7-1-5}$ & 0.72 & 0.0001 & \\
\hline
\end{tabular}




\begin{tabular}{|c|c|c|c|c|}
\hline \multirow{5}{*}{$\mathrm{IMR}_{2-7-1} \rightarrow \mathrm{IM}_{\mathrm{R} 2-6-1}$} & $\mathrm{TS}_{\mathrm{R} 2-7-1-1}$ & $1.4 \times 10^{4}$ & 0.2697 & $4.7 \times 10^{3}$ \\
\hline & $\mathrm{TS}_{\mathrm{R} 2-7-1-2}$ & $1.7 \times 10^{4}$ & 0.0547 & \\
\hline & $\mathrm{TS}_{\mathrm{R} 2-7-1-3}$ & $2.8 \times 10^{5}$ & 0.0002 & \\
\hline & $\mathrm{TS}_{\mathrm{R} 2-7-1-4}$ & $2.2 \times 10^{3}$ & 0.0039 & \\
\hline & $\mathrm{TS}_{\mathrm{R} 2-7-1-5}$ & $4.2 \times 10^{5}$ & 0.0001 & \\
\hline \multirow[t]{4}{*}{$\mathrm{IM}_{\mathrm{R} 2-6-1} \rightarrow \mathrm{IM}_{\mathrm{R} 2-7-2}$} & $\mathrm{TS}_{\mathrm{R} 2-7-2-1}$ & $3.8 \times 10^{-4}$ & 0.0742 & $5.5 \times 10^{-5}$ \\
\hline & $\mathrm{TS}_{\mathrm{R} 2-7-2-2}$ & $7.1 \times 10^{-5}$ & 0.0344 & \\
\hline & $\mathrm{TS}_{\mathrm{R} 2-7-2-3}$ & $7.2 \times 10^{-4}$ & 0.0291 & \\
\hline & $\mathrm{TS}_{\mathrm{R} 2-7-2-4}$ & $8.4 \times 10^{-4}$ & 0.0043 & \\
\hline \multirow{4}{*}{$\mathrm{IM}_{\mathrm{R} 2-7-2} \rightarrow \mathrm{IM}_{\mathrm{R} 2-6-1}$} & $\mathrm{TS}_{\mathrm{R} 2-7-2-1}$ & $4.5 \times 10^{5}$ & 0.1848 & $1.7 \times 10^{5}$ \\
\hline & $\mathrm{TS}_{\mathrm{R} 2-7-2-2}$ & $2.0 \times 10^{5}$ & 0.0109 & \\
\hline & $\mathrm{TS}_{\mathrm{R} 2-7-2-3}$ & $4.5 \times 10^{5}$ & 0.1517 & \\
\hline & $\mathrm{TS}_{\mathrm{R} 2-7-2-4}$ & $1.9 \times 10^{5}$ & 0.0800 & \\
\hline \multirow[t]{5}{*}{$\mathrm{IM}_{\mathrm{R} 2-8-1} \rightarrow \mathrm{P}_{\mathrm{R} 2-9-1}$} & $\mathrm{TS}_{\mathrm{R} 2-9-1-1}$ & 0.78 & 0.2241 & 0.23 \\
\hline & $\mathrm{TS}_{\mathrm{R} 2-9-1-2}$ & 0.15 & 0.0206 & \\
\hline & $\mathrm{TS}_{\mathrm{R} 2-9-1-3}$ & 4.9 & 0.0089 & \\
\hline & $\mathrm{TS}_{\mathrm{R} 2-9-1-4}$ & 0.26 & 0.0082 & \\
\hline & $\mathrm{TS}_{\mathrm{R} 2-9-1-5}$ & 16.4 & 0.0001 & \\
\hline \multirow[t]{3}{*}{$\mathrm{IM}_{\mathrm{R} 2-8-1} \rightarrow \mathrm{P}_{\mathrm{R} 2-9-2}$} & $\mathrm{TS}_{\mathrm{R} 2-9-2-1}$ & $4.3 \times 10^{-5}$ & 0.0042 & $3.9 \times 10^{-6}$ \\
\hline & $\mathrm{TS}_{\mathrm{R} 2-9-2-2}$ & $6.8 \times 10^{-6}$ & 0.0010 & \\
\hline & $\mathrm{TS}_{\mathrm{R} 2-9-2-3}$ & $4.4 \times 10^{-3}$ & 0.0008 & \\
\hline \multirow[t]{4}{*}{$\mathrm{TMP}-\mathrm{RO} \rightarrow \mathrm{P}_{\mathrm{R} 1-4-1}$} & $\mathrm{TS}_{\mathrm{R} 1-4-1-1}$ & $5.4 \times 10^{6}$ & 0.1832 & $3.0 \times 10^{6}$ \\
\hline & $\mathrm{TS}_{\mathrm{R} 1-4-1-2}$ & $2.7 \times 10^{6}$ & 0.1504 & \\
\hline & $\mathrm{TS}_{\mathrm{R} 1-4-1-3}$ & $1.1 \times 10^{7}$ & 0.0870 & \\
\hline & $\mathrm{TS}_{\mathrm{R} 1-4-1-4}$ & $8.9 \times 10^{6}$ & 0.0797 & \\
\hline \multirow[t]{2}{*}{$\mathrm{TMP}-\mathrm{RO} \rightarrow \mathrm{P}_{\mathrm{R} 1-4-2}$} & $\mathrm{TS}_{\mathrm{R} 1-4-2-1}$ & $3.5 \times 10^{2}$ & 0.0639 & 24.9 \\
\hline & $\mathrm{TS}_{\mathrm{R} 1-4-2-2}$ & 95.4 & 0.0234 & \\
\hline \multirow[t]{3}{*}{$\mathrm{TCPP}-\mathrm{RO} \rightarrow \mathrm{P}_{\mathrm{R} 2-10-1}$} & $\mathrm{TS}_{\mathrm{R} 2-10-1-1}$ & $2.3 \times 10^{7}$ & 0.0018 & $5.3 \times 10^{4}$ \\
\hline & $\mathrm{TS}_{\mathrm{R} 2-10-1-2}$ & $1.7 \times 10^{7}$ & 0.0005 & \\
\hline & $\mathrm{TS}_{\mathrm{R} 2-10-1-3}$ & $1.4 \times 10^{7}$ & 0.0002 & \\
\hline \multirow[t]{3}{*}{$\mathrm{TCPP}-\mathrm{RO} \rightarrow \mathrm{P}_{\mathrm{R} 2-10-2}$} & $\mathrm{TS}_{\mathrm{R} 2-10-2-1}$ & $2.6 \times 10^{3}$ & 0.2244 & $6.3 \times 10^{2}$ \\
\hline & $\mathrm{TS}_{\mathrm{R} 2-10-2-2}$ & $1.9 \times 10^{2}$ & 0.2018 & \\
\hline & $\mathrm{TS}_{\mathrm{R} 2-10-2-3}$ & $1.9 \times 10^{3}$ & 0.0061 & \\
\hline
\end{tabular}


205 from TMP-R $\cdot+\mathbf{O}_{2}$ and TCPP-R $\cdot+\mathbf{O}_{2}$ reactions
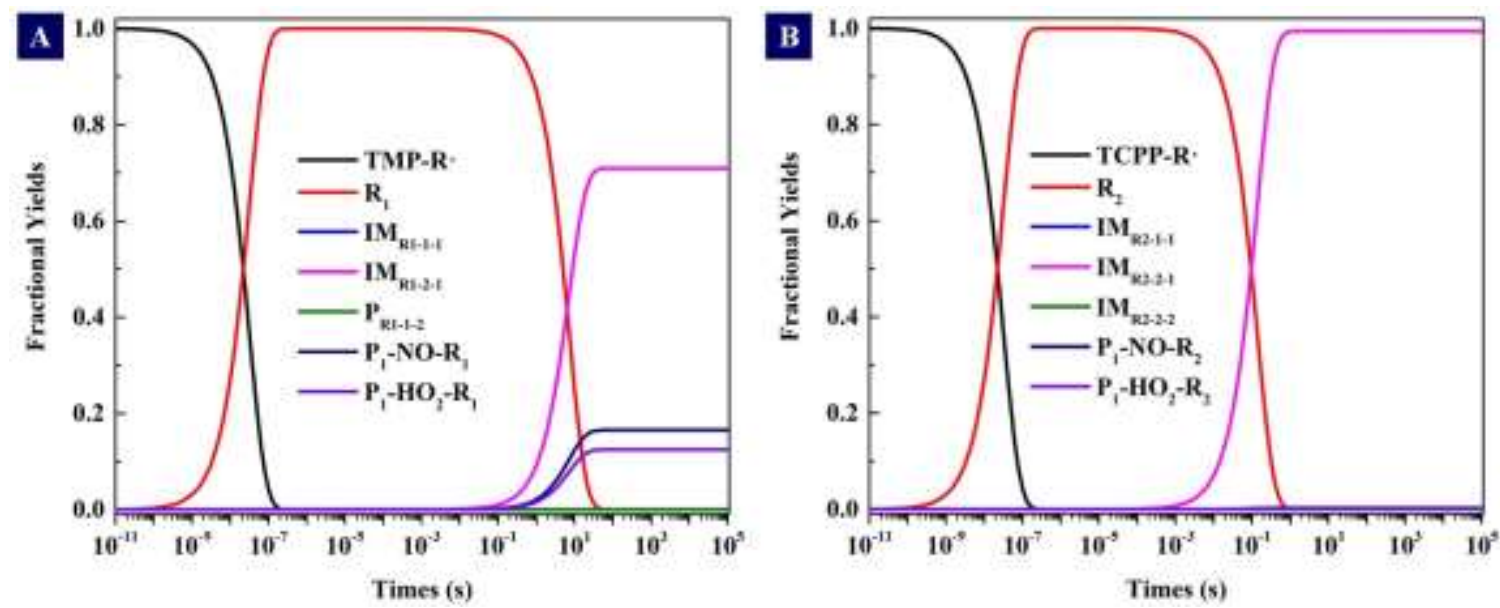

207 Figure S6. Calculated time-dependent fractional yields of main transformation products

208 from the reactions of TMP-R · (A) and TCPP-R - (B) with $\mathrm{O}_{2}$ (under $100 \mathrm{ppt}$ NO and $40 \mathrm{ppt}$

$209 \mathrm{HO}_{2}$ conditions, at $298 \mathrm{~K}$ and $\left.1 \mathrm{~atm}\right)$. The symbols " $\mathrm{P}_{1}-\mathrm{NO}-\mathrm{R}_{1 / 2}$ " and " $\mathrm{P}_{1}-\mathrm{HO}_{2}-\mathrm{R}_{1 / 2}$ "

210 represent the products from reactions of $\mathrm{R}_{1} / \mathrm{R}_{2}$ with $\mathrm{NO}$ and $\mathrm{HO}_{2} \cdot$, respectively. 
211 S7. Schematic potential energy surfaces of the various $\mathbf{C}$-center radicals with $\mathbf{O}_{2}$

\section{2 involved in $\mathbf{R}_{2}$ reactions}

213

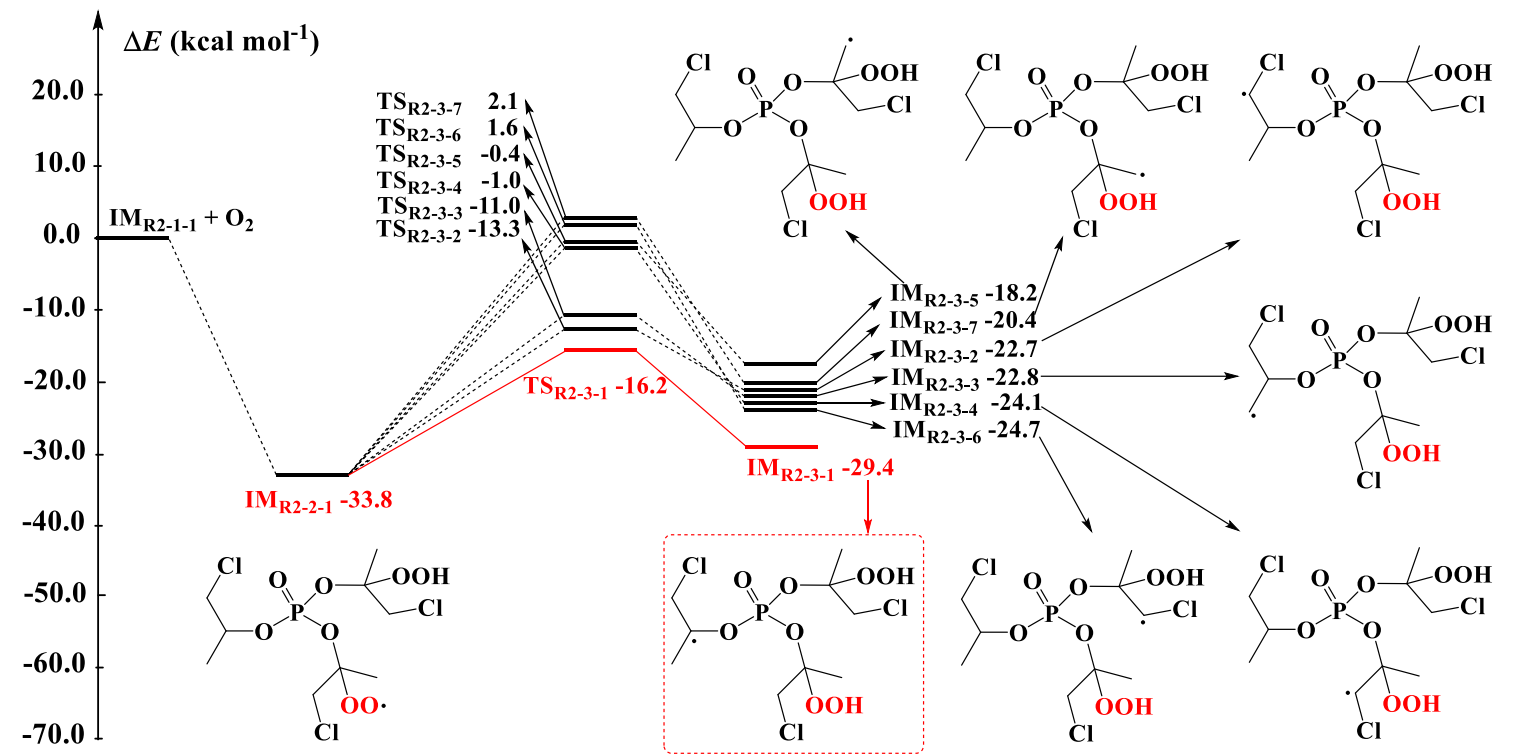

214 Figure S7. Schematic potential energy surfaces for $\mathrm{IM}_{\mathrm{R} 2-1-1}+\mathrm{O}_{2}$ reaction at the M06-2X/6-

$215311+\mathrm{G}(3 \mathrm{df}, 2 \mathrm{p}) / / \mathrm{M} 06-2 \mathrm{X} / 6-31+\mathrm{G}(\mathrm{d}, \mathrm{p})$ level of theory. The symbols $\mathrm{IM}_{\mathrm{R} 2-n-m}$ and TS $\mathrm{R} 2 \square n-m_{n}$

216 represent the intermediates and transition states, respectively; $n$ and $m$ denote different

217 species. The energies of the $\mathrm{IM}_{\mathrm{R} 2-1-1}+\mathrm{O}_{2}$ are set to zero (reference state) for the reactions 218 of IMR2-2-1. 


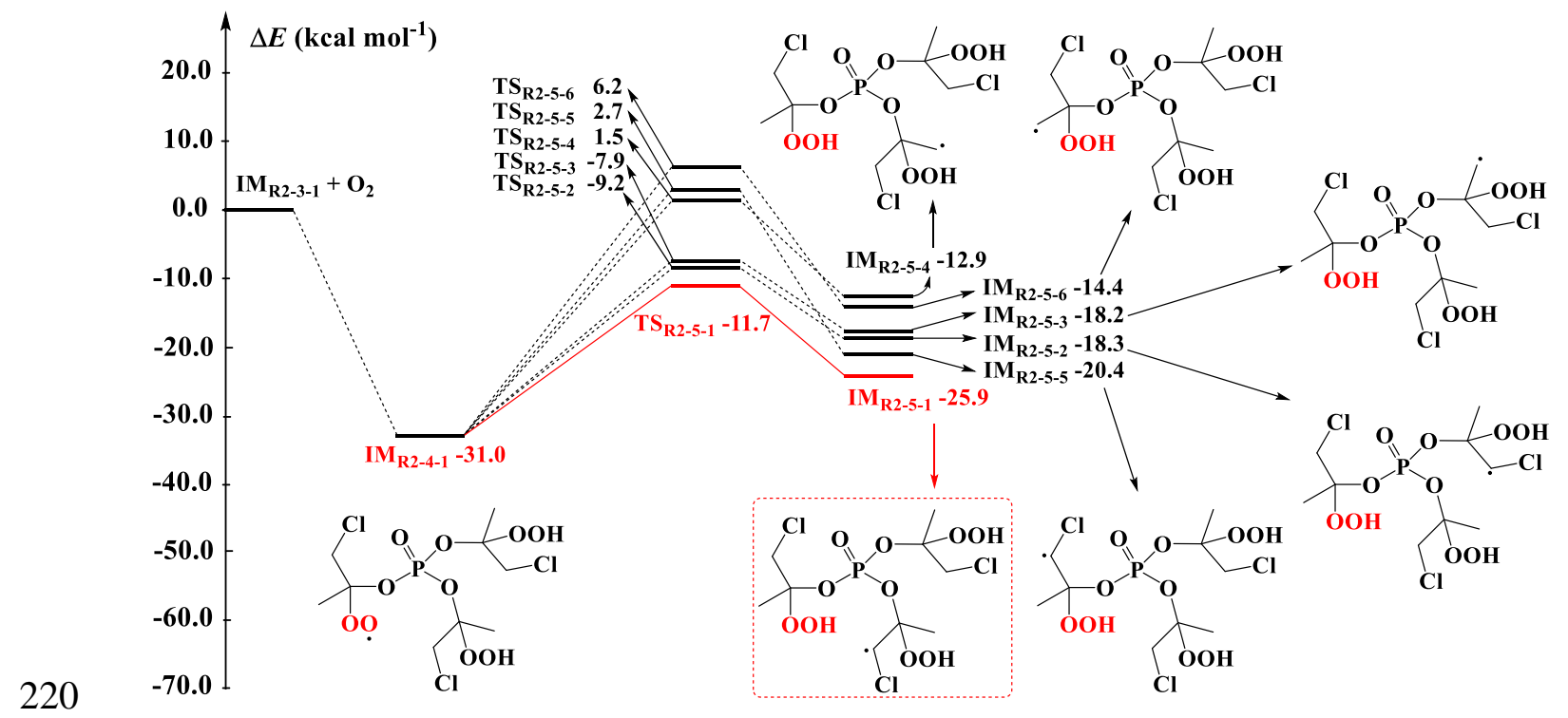

221 Figure S8. Schematic potential energy surfaces for $\mathrm{IM}_{\mathrm{R} 2-3-1}+\mathrm{O}_{2}$ reaction at the M06-2X/6-

$222311+\mathrm{G}(3 \mathrm{df}, 2 \mathrm{p}) / / \mathrm{M} 06-2 \mathrm{X} / 6-31+\mathrm{G}(\mathrm{d}, \mathrm{p})$ level of theory. The symbols $\mathrm{IM}_{\mathrm{R} 2-n-m}$ and TS $2 \square n-m$

223 represent the intermediates and transition states, respectively; $n$ and $m$ denote different

224 species. The energies of the $\mathrm{IM}_{\mathrm{R} 2-3-1}+\mathrm{O}_{2}$ are respectively set to zero (reference state) for 225 the reactions of $\mathrm{IM}_{\mathrm{R} 2-4-1 .}$ 


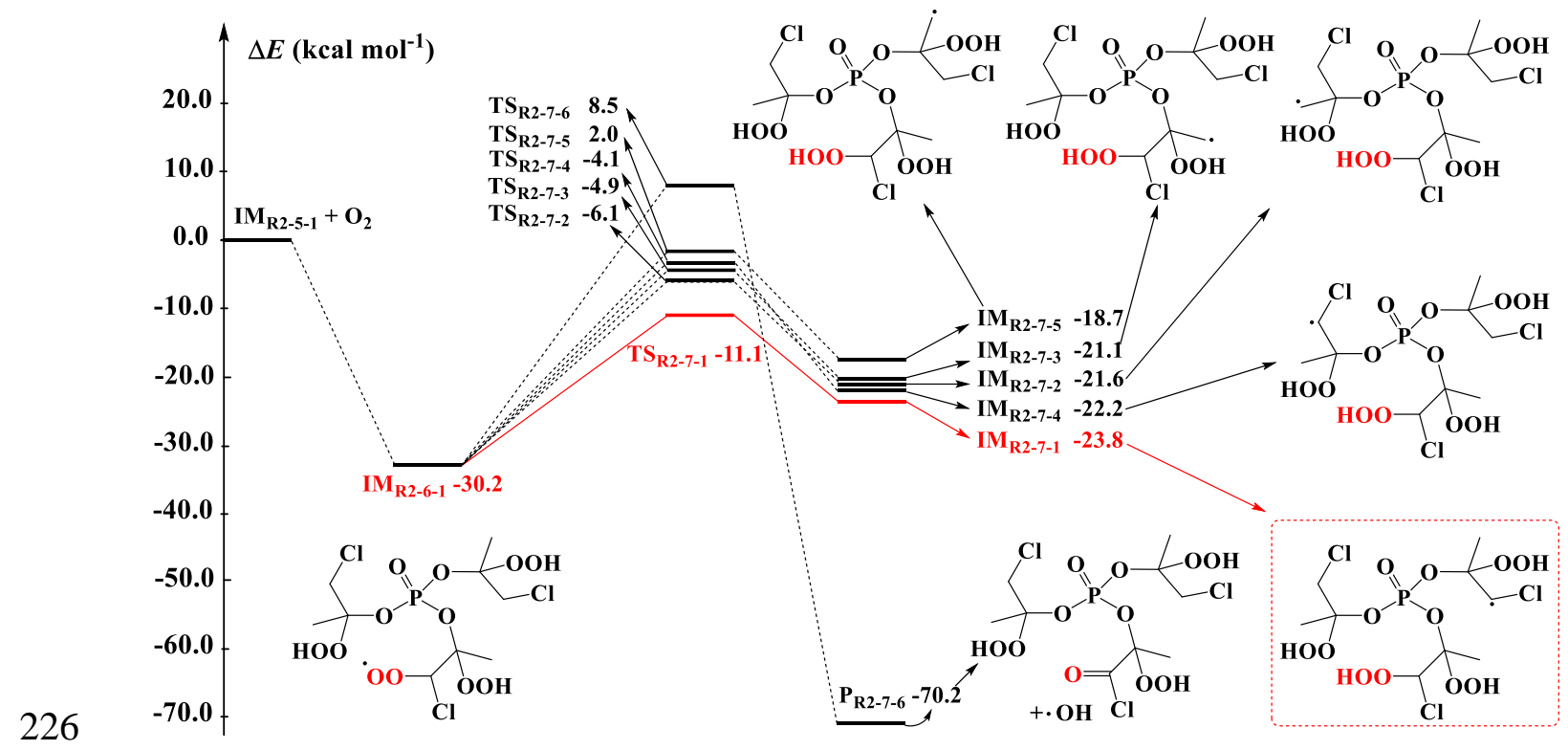

227 Figure S9. Schematic potential energy surfaces for $\mathrm{IM}_{\mathrm{R} 2-5-1}+\mathrm{O}_{2}$ reaction at the M06-2X/6-

$228311+\mathrm{G}(3 \mathrm{df}, 2 \mathrm{p}) / / \mathrm{M} 06-2 \mathrm{X} / 6-31+\mathrm{G}(\mathrm{d}, \mathrm{p})$ level of theory. The symbols $\mathrm{IM}_{\mathrm{R} 2-n-m}, \mathrm{TS}_{\mathrm{R} 2 \square n-m}$ and

$229 \mathrm{P}_{\mathrm{R} 2 \square n-m}$ represent the intermediates, transition states and products, respectively; $n$ and $m$

230 denote different species. The energies of the $\mathrm{IM}_{\mathrm{R} 2-5-1}+\mathrm{O}_{2}$ are respectively set to zero

231 (reference state) for the reactions of $\mathrm{IM}_{\mathrm{R} 2-6-1}$. 


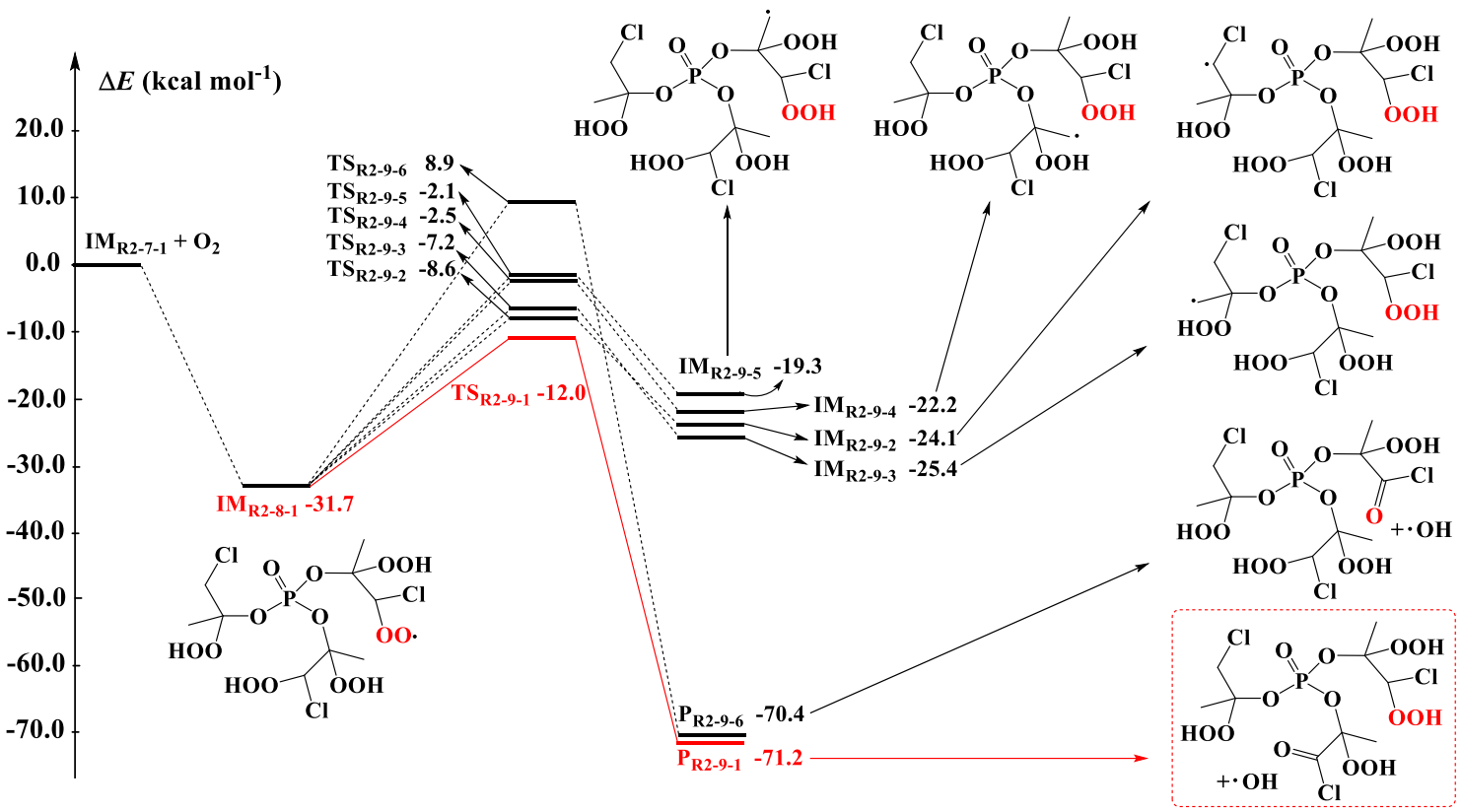

233 Figure S10. Schematic potential energy surfaces for $\mathrm{IM}_{\mathrm{R} 2-7-1}+\mathrm{O}_{2}$ reaction at the M06-

$2342 \mathrm{X} / 6-311+\mathrm{G}(3 \mathrm{df}, 2 \mathrm{p}) / / \mathrm{M} 06-2 \mathrm{X} / 6-31+\mathrm{G}(\mathrm{d}, \mathrm{p})$ level of theory. The symbols $\mathrm{IM}_{\mathrm{R} 2-n-m}, \mathrm{TS}_{\mathrm{R} 2 \square n-}$

$235 m$ and $\mathrm{P}_{\mathrm{R} 2 \square n-m}$ represent the intermediates, transition states and products, respectively; $n$ and

$236 m$ denote different species. The energies of the $\mathrm{IM}_{\mathrm{R} 2-7-1}+\mathrm{O}_{2}$ are respectively set to zero

237 (reference state) for the reactions of $\mathrm{IM}_{\mathrm{R} 2-8-1 .}$ 
238 S8. The Lennard-Jones parameters of intermediates

239 Table S4. Lennard-Jones parameters of intermediates for various reactions used in the 240 MESMER simulations.

\begin{tabular}{lcc}
\hline Species & $\sigma /(\AA)$ & $\varepsilon /(\mathrm{K})$ \\
\hline TMP-R & 6.2 & 498 \\
$\mathrm{R}_{1}$ & 6.5 & 552 \\
$\mathrm{IM}_{\mathrm{R} 1-2-1}$ & 6.7 & 606 \\
\hline $\mathrm{TCPP}-\mathrm{R} \cdot$ & 8.4 & 769 \\
$\mathrm{R}_{2}$ & 8.5 & 823 \\
$\mathrm{IM}_{\mathrm{R} 2-2-1}$ & 8.6 & 877 \\
$\mathrm{IM}_{\mathrm{R} 2-4-1}$ & 8.8 & 931 \\
$\mathrm{IM}_{\mathrm{R} 2-6-1}$ & 8.9 & 986 \\
$\mathrm{IM}_{\mathrm{R} 2-8-1}$ & 9.0 & 1040 \\
\hline
\end{tabular}

241 
243 In principle, both electronic and steric effects could be the reasons that the $-(\mathrm{O})_{3} \mathrm{P}(=\mathrm{O})$

244 functional group can facilitate the occurrence of $\mathrm{H}$-shift reaction. The electronic effect

245 could affect the $\mathrm{H}$-shift reaction mainly via changing the $\mathrm{C}-\mathrm{H}$ bond energy (which $\mathrm{H}$ is

246 shifted) and the radical activity of peroxy radicals. By comparing the C-H energy of TMP

247 (96.6 kcal $/ \mathrm{mol})$ and an alkane (here butane was selected, $98.2 \mathrm{kcal} / \mathrm{mol})$, it can be

248 concluded that the introduction of the $-(\mathrm{O})_{3} \mathrm{P}(=\mathrm{O})$ functional group can decrease its

249 adjacent $\mathrm{C}-\mathrm{H}$ bond energy and therefore facilitate the occurrence of the $\mathrm{H}$-shift reaction,

250 compared to the alkane. The change of radical activity of peroxy radicals caused by the -

$251(\mathrm{O})_{3} \mathrm{P}(=\mathrm{O})$ functional group could be evaluated by comparing the $1,7 \mathrm{H}$-shift reaction

252 energy barrier of selected model compounds 1 with 2 , and 3 with 4 . As can be seen in

253 Figure S11, the 1,7 H-shift reaction energy barriers of model compounds 1 and 3 with

254 additional $-(\mathrm{O}){ }_{3} \mathrm{P}(=\mathrm{O})$ functional group on the adjacent site of the $-\mathrm{OO} \cdot$ group are lower

255 than that of compounds 2 and 4, respectively. This implies that the introduction of -

$256(\mathrm{O})_{3} \mathrm{P}(=\mathrm{O})$ group on the adjacent site of the -OO group could increase the activity of

257 abstracting an $\mathrm{H}$-atom. Therefore, the decrease of the $\mathrm{C}-\mathrm{H}$ bond energy (where $\mathrm{H}$ is shifted)

258 and the increase of the radical activity of peroxy radicals should be the main electronic

259 effect caused by $-(\mathrm{O}){ }_{3} \mathrm{P}(=\mathrm{O})$ functional group.

260 In addition, since H-shift reaction energy barrier via cyclic transition state arching 
261 across the $-(\mathrm{O})_{3} \mathrm{P}(=\mathrm{O})$ functional group is always lower than that via the cyclic transition

262 state without arching across the $-(\mathrm{O})_{3} \mathrm{P}(=\mathrm{O})$ group. This imply that the steric effect caused

263 by the $-(\mathrm{O})_{3} \mathrm{P}(=\mathrm{O})$ functional group could facilitate the occurrence of $\mathrm{H}$-shift reaction. We

264 attempted to unveil the steric effect by comparing the reaction energy barriers of $1,7 \mathrm{H}$ -

265 shifts of model compound 1 with $\mathrm{TMP}-\mathrm{RO}_{2}$, and compound 3 with $\mathrm{TCPP}-\mathrm{RO}_{2} \cdot($ see

266 Figure S11). As can be seen in Figure S11, the reaction energy barriers of 1,7 H-shifts of

$267 \mathrm{TMP}-\mathrm{RO}_{2} \cdot$ and TCPP- $\mathrm{RO}_{2} \cdot$ are lower than that of model compound 1 and 3 , respectively,

268 indicating the steric effect of the $-(\mathrm{O})_{3} \mathrm{P}(=\mathrm{O})$ group on facilitating the occurrence of $\mathrm{H}$-shift

269 reaction. Therefore, the electronic (including the decrease of the $\mathrm{C}-\mathrm{H}$ bond energy and the

270 increase of the radical reactivity of peroxy radicals) and steric effects could be the main

271 reason for the role of $-(\mathrm{O})_{3} \mathrm{P}(=\mathrm{O})$ group in facilitating the $\mathrm{H}$-shift reactions. 

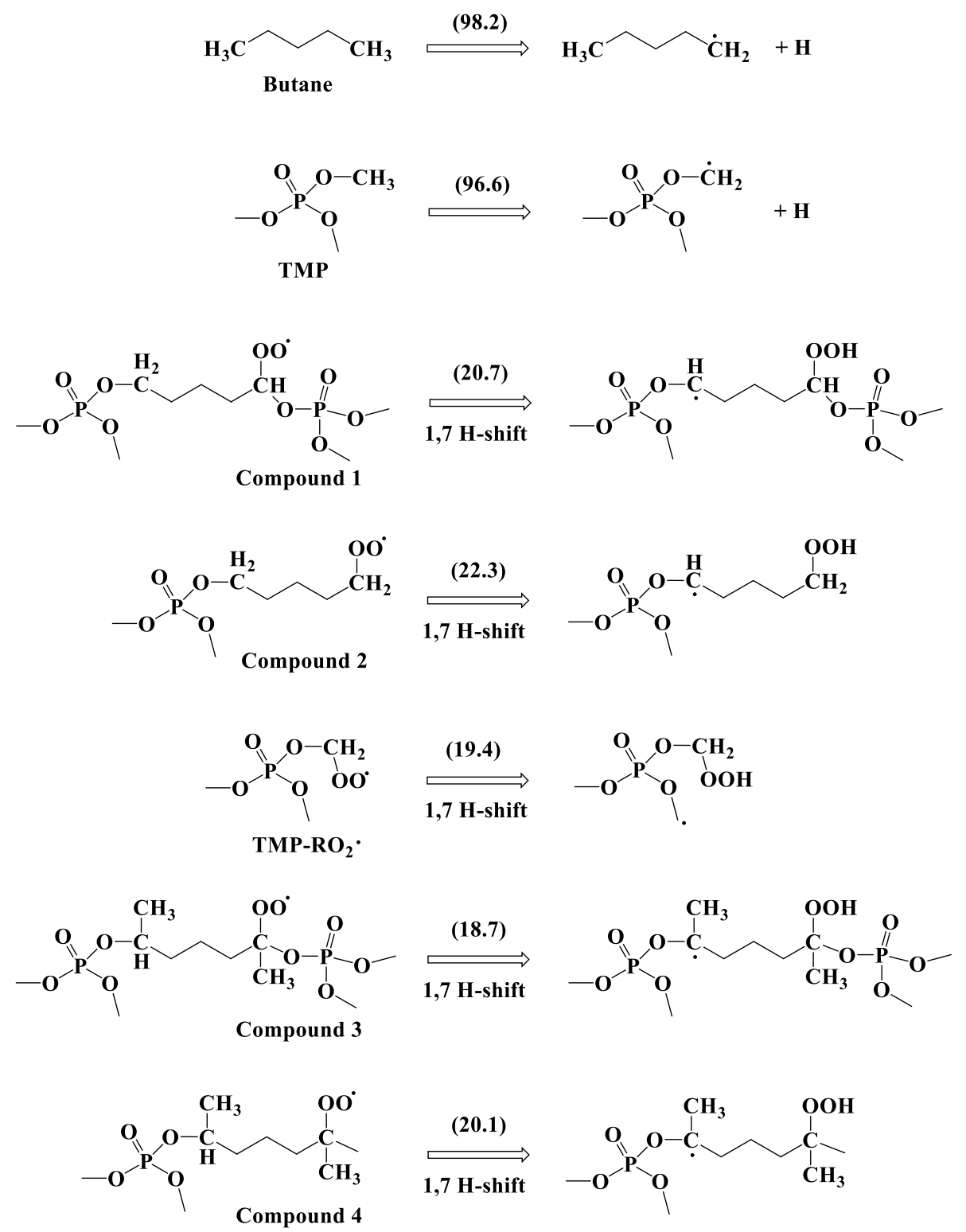

273 Figure S11. Calculated C-H bond energies of TMP and butane, and H-shift reaction energy

274 barriers of selected model $\mathrm{RO}_{2}$. at the M06-2X/6-311+G(3df,2p)//M06-2X/6-31+G(d,p)

275 level of theory. All energetic information is shown in the bracket (in $\mathrm{kcal} \mathrm{mol}^{-1}$ ). 
$276 \mathrm{S10}$. $\mathrm{H}$-shift reactions of $\mathrm{RO}_{2} \cdot$ with the $-(\mathrm{O})_{3} \mathrm{~B},-(\mathrm{O})_{2} \mathrm{~S}(=\mathrm{O})_{2}$ and $-\mathrm{OS}(=\mathrm{O})_{2}(\mathrm{OH})$ ester

277 functional groups
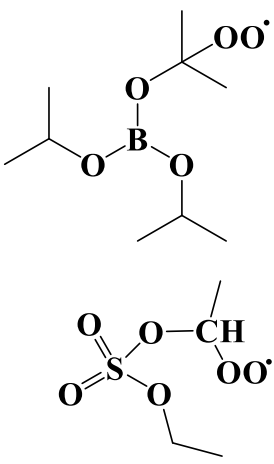

278

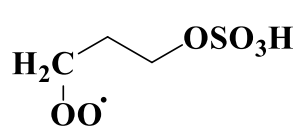

(18.7)

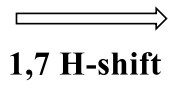

(18.3)
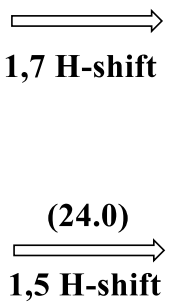
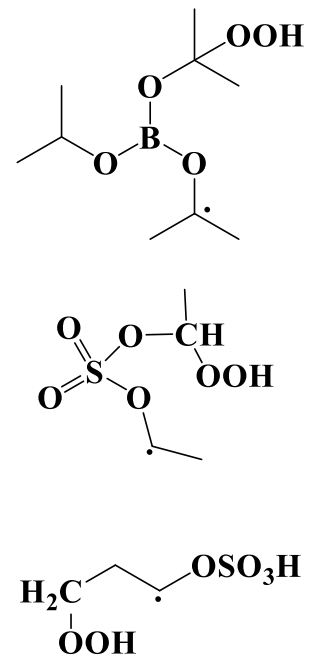

279 Figure S12. Calculated $\mathrm{H}$-shift reaction energy barriers of $\mathrm{RO}_{2}$. with the $-(\mathrm{O})_{3} \mathrm{~B}$, -

$280 \quad(\mathrm{O})_{2} \mathrm{~S}(=\mathrm{O})_{2}$ and $-\mathrm{OS}(=\mathrm{O})_{2}(\mathrm{OH})$ ester functional groups at the M06-2X/6-

$281311+\mathrm{G}(3 \mathrm{df}, 2 \mathrm{p}) / / \mathrm{M} 06-2 \mathrm{X} / 6-31+\mathrm{G}(\mathrm{d}, \mathrm{p})$ level of theory. All energetic information is shown 282 in the brackets (in $\mathrm{kcal} \mathrm{mol}^{-1}$ ). 
283 S11. Toxicity assessment of products formed in atmospheric transformation of $R_{1}$ and

$284 \quad \mathbf{R}_{2}$

285

Table S5. The acute and chronic toxicity classification $(\mathrm{mg} / \mathrm{L})$

\begin{tabular}{lll}
\hline Classification & Acute toxicity & Chronic toxicity \\
\hline Very toxic & LC50 $<1$ or EC $50<1$ & $\mathrm{ChV}<0.1$ \\
Toxic & $1<\mathrm{LC} 50<10$ or $1<$ EC50 $<10$ & $0.1<\mathrm{ChV}<1$ \\
Harmful & $10<\mathrm{LC} 50<100$ or $10<\mathrm{EC}_{50}<100$ & $1<\mathrm{ChV}<10$ \\
Not harmful & $\mathrm{LC} 50>100$ or EC $50>100$ & $\mathrm{ChV}>10$ \\
\hline
\end{tabular}



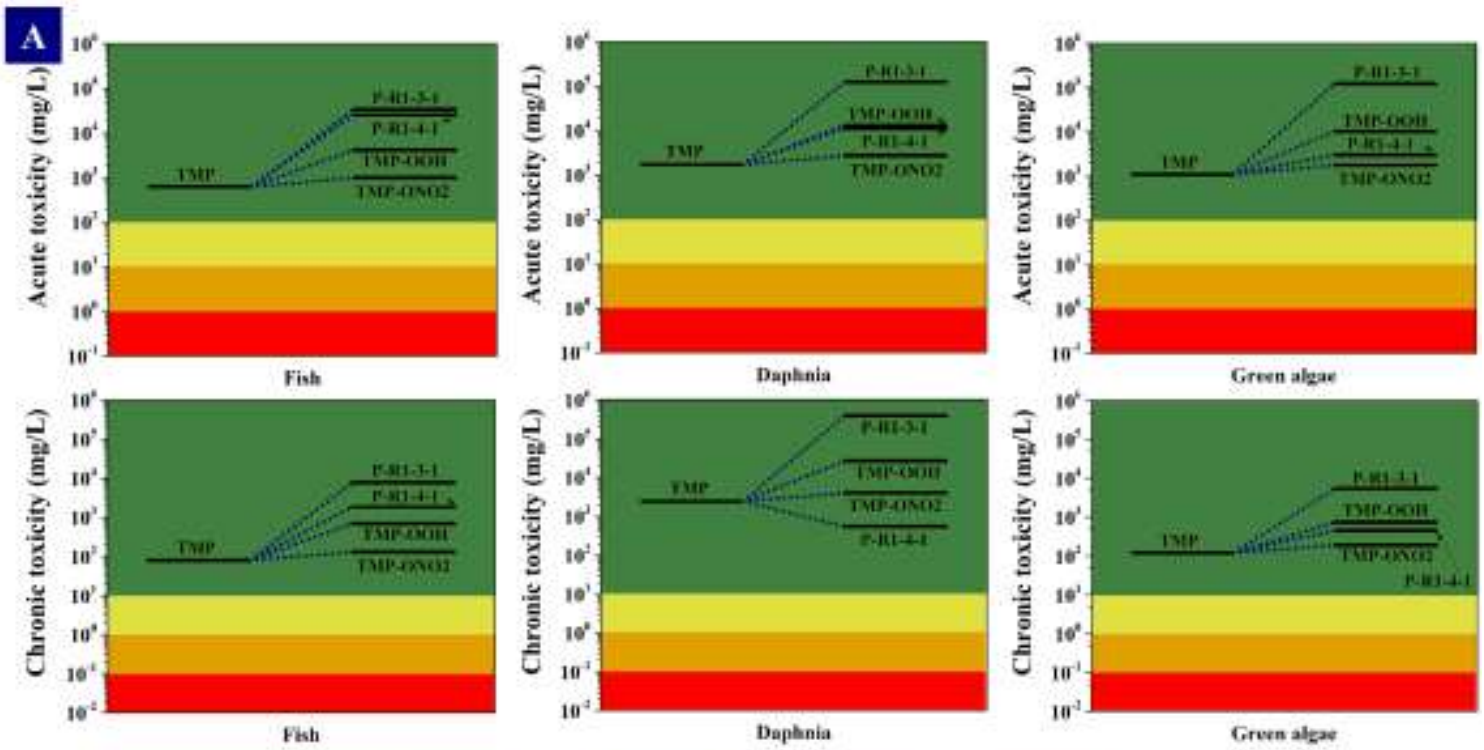

287

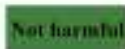

Itarmfol
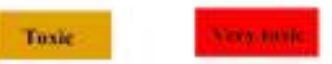

Green alkae
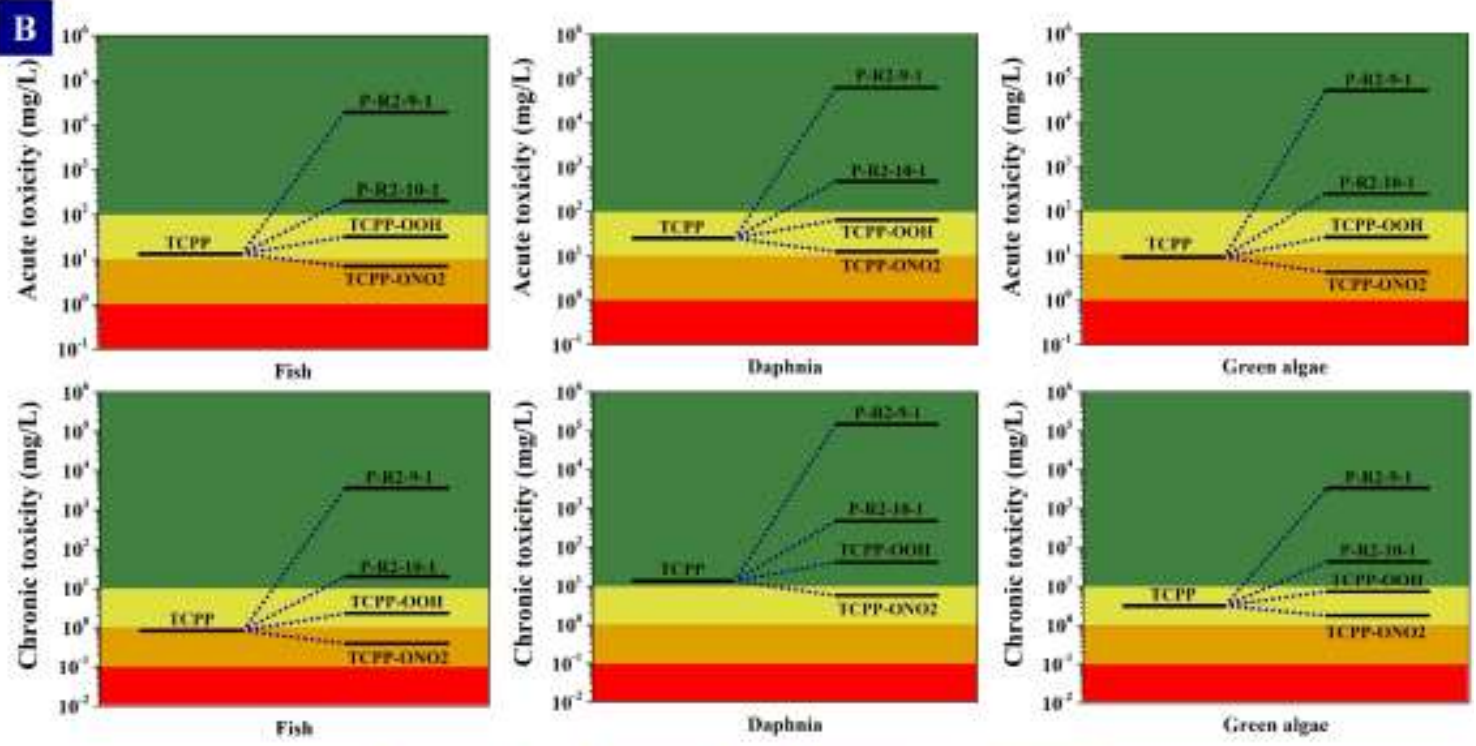

289 Figure S13. Acute and chronic toxicities for TMP, TCPP and transformation products of $\mathrm{R}_{1}$

290 and $\mathrm{R}_{2}$ for fish, daphnia and green algae. 
291 S12. Cartesian coordinates of the transition states for all reactions

292 Table S6. Cartesian coordinates of the transition states for all reactions.

\begin{tabular}{|c|c|c|c|c|}
\hline Species & Atoms & $X$ & Y & $\mathrm{Z}$ \\
\hline \multirow[t]{18}{*}{$\mathrm{TS}_{\mathrm{R} 1-1-1}$} & $\mathrm{P}$ & -0.7038069650 & -0.0311529626 & 0.4204158556 \\
\hline & $\mathrm{O}$ & 0.1586938286 & 1.2707152553 & 0.8078351124 \\
\hline & $\mathrm{O}$ & 0.1490296972 & -0.7471978624 & -0.7495535360 \\
\hline & $\mathrm{O}$ & -1.8810874813 & 0.6169795549 & -0.4249430005 \\
\hline & $\mathrm{C}$ & 1.2947573750 & -1.5011747210 & -0.3807615534 \\
\hline & $\mathrm{H}$ & 1.0314085596 & -2.2903659692 & 0.3284426066 \\
\hline & $\mathrm{H}$ & 1.7157043191 & -1.8783527272 & -1.3131601402 \\
\hline & $\mathrm{C}$ & -2.9441874323 & -0.2362934652 & -0.8813202934 \\
\hline & $\mathrm{H}$ & -3.6887723852 & 0.4213902363 & -1.3260752376 \\
\hline & $\mathrm{H}$ & -2.5639701190 & -0.9341930036 & -1.6315583992 \\
\hline & $\mathrm{H}$ & -3.3756127916 & -0.7799235101 & -0.0379463935 \\
\hline & $\mathrm{C}$ & 0.8890569225 & 1.9522283102 & -0.1566647193 \\
\hline & $\mathrm{H}$ & 1.3151270779 & 2.8534087775 & 0.2787218197 \\
\hline & $\mathrm{H}$ & 1.9249214830 & 1.1672599069 & -0.4442425917 \\
\hline & $\mathrm{H}$ & 0.3692007610 & 2.0793252391 & -1.1090864169 \\
\hline & $\mathrm{O}$ & -1.0520668606 & -0.8853049027 & 1.5594676839 \\
\hline & $\mathrm{O}$ & 2.2183496631 & -0.6994073592 & 0.3013222119 \\
\hline & $\mathrm{O}$ & 2.7072223483 & 0.2603072028 & -0.5711570085 \\
\hline \multirow[t]{14}{*}{$\mathrm{TS}_{\mathrm{R} 1-1-2}$} & $\mathrm{P}$ & 0.7717646429 & -0.3387731060 & 0.4638001678 \\
\hline & $\mathrm{O}$ & 1.8507116145 & -0.8768764342 & -0.5648359558 \\
\hline & $\mathrm{O}$ & -0.5770199285 & -0.4158746200 & -0.4626383583 \\
\hline & $\mathrm{O}$ & 1.0944381714 & 1.2046852013 & 0.5499043406 \\
\hline & $\mathrm{C}$ & -1.7964160170 & -0.2905766629 & 0.1653646115 \\
\hline & $\mathrm{H}$ & -1.9662582924 & -0.9787039413 & 0.9985039453 \\
\hline & $\mathrm{H}$ & -2.0255774507 & 0.9486880894 & 0.5464188289 \\
\hline & $\mathrm{C}$ & 1.2116637609 & 1.9948696840 & -0.6479165437 \\
\hline & $\mathrm{H}$ & 1.3765022396 & 3.0175386244 & -0.3141553178 \\
\hline & $\mathrm{H}$ & 2.0603407090 & 1.6458273097 & -1.2390680292 \\
\hline & $\mathrm{H}$ & 0.2885668760 & 1.9311347613 & -1.2297181227 \\
\hline & $\mathrm{C}$ & 1.8874850978 & -2.2855799894 & -0.8541921145 \\
\hline & $\mathrm{H}$ & 2.7613915911 & -2.4409368865 & -1.4839987895 \\
\hline & $\mathrm{H}$ & 1.9817719577 & -2.8546943447 & 0.0730143800 \\
\hline
\end{tabular}




\begin{tabular}{|c|c|c|c|c|}
\hline & $\mathrm{H}$ & 0.9813192205 & -2.5752616661 & -1.3918611105 \\
\hline & $\mathrm{O}$ & 0.6146694861 & -1.0245080701 & 1.7508880083 \\
\hline & $\mathrm{O}$ & -2.8233419973 & -0.1358587869 & -0.7137061825 \\
\hline & $\mathrm{O}$ & -2.9267216815 & 1.2822968379 & -0.3358277577 \\
\hline \multirow[t]{18}{*}{$\mathrm{TS}_{\mathrm{R} 1-1-3}$} & $\mathrm{P}$ & -2.9854035601 & 0.5221798506 & -0.3414996799 \\
\hline & $\mathrm{O}$ & -2.7780833735 & -1.0457709227 & -0.2877536831 \\
\hline & $\mathrm{O}$ & -1.1341892077 & 0.5456213757 & 0.6756210102 \\
\hline & $\mathrm{O}$ & -4.5842843389 & 0.3759978935 & -0.4853887706 \\
\hline & $\mathrm{C}$ & -1.0474540448 & 1.7092962325 & 1.2259852740 \\
\hline & $\mathrm{H}$ & -0.4709004465 & 1.7575106649 & 2.1713210196 \\
\hline & $\mathrm{H}$ & -0.6916294736 & 2.5249635740 & 0.5500177039 \\
\hline & $\mathrm{C}$ & -5.3461302377 & 1.5368133365 & -0.8230981607 \\
\hline & $\mathrm{H}$ & -4.9164760312 & 2.0307455537 & -1.6979424940 \\
\hline & $\mathrm{H}$ & -6.3523673443 & 1.1828717068 & -1.0455122204 \\
\hline & $\mathrm{H}$ & -5.3780359725 & 2.2322198923 & 0.0204696555 \\
\hline & $\mathrm{C}$ & -1.5301962844 & -1.7001028967 & -0.5505952871 \\
\hline & $\mathrm{H}$ & -1.7966417823 & -2.6611580249 & -0.9898733425 \\
\hline & $\mathrm{H}$ & -0.9265771951 & -1.1156933596 & -1.2471993273 \\
\hline & $\mathrm{H}$ & -0.9872241296 & -1.8385677617 & 0.3835667130 \\
\hline & $\mathrm{O}$ & -2.4150609468 & 1.4377821689 & -1.3533006992 \\
\hline & $\mathrm{O}$ & -2.3575817819 & 2.2068145429 & 1.6301243419 \\
\hline & $\mathrm{O}$ & -3.3081022088 & 1.3676211734 & 1.2013101769 \\
\hline \multirow[t]{14}{*}{$\mathrm{TS}_{\mathrm{R} 1-3-1}$} & $\mathrm{P}$ & 0.8109942825 & 0.1098219821 & 0.3456193814 \\
\hline & $\mathrm{O}$ & -0.0687967316 & -0.9327869627 & 1.1602185306 \\
\hline & $\mathrm{O}$ & -0.0565055246 & 0.4865524269 & -0.9797648277 \\
\hline & $\mathrm{O}$ & 1.9571358543 & -0.7874654583 & -0.2737995645 \\
\hline & $\mathrm{C}$ & -1.3731636673 & 0.9023935888 & -0.8250915667 \\
\hline & $\mathrm{H}$ & -1.7172615801 & 1.3751607295 & -1.7469735717 \\
\hline & $\mathrm{H}$ & -2.0456183862 & -0.2309327376 & -0.6515429304 \\
\hline & $\mathrm{C}$ & 2.9886913030 & -0.1613325924 & -1.0607382673 \\
\hline & $\mathrm{H}$ & 3.4582521631 & 0.6401715834 & -0.4868896583 \\
\hline & $\mathrm{H}$ & 3.7117768898 & -0.9433567577 & -1.2829143920 \\
\hline & $\mathrm{H}$ & 2.5589519728 & 0.2287676989 & -1.9862901379 \\
\hline & $\mathrm{H}$ & -0.1366706636 & 2.6466702287 & 0.7386875070 \\
\hline & $\mathrm{O}$ & 1.2152184786 & 1.2961063959 & 1.1195195444 \\
\hline & $\mathrm{O}$ & -1.6316920319 & 1.5792597383 & 0.3307986222 \\
\hline
\end{tabular}




\begin{tabular}{|c|c|c|c|c|}
\hline & $\mathrm{O}$ & -0.9664134461 & 2.8562605330 & 0.2570202943 \\
\hline & $\mathrm{C}$ & -0.4858059071 & -2.1835215124 & 0.6249470573 \\
\hline & $\mathrm{H}$ & -1.1659498020 & -2.5995272085 & 1.3678885728 \\
\hline & $\mathrm{H}$ & 0.3789358113 & -2.8199053681 & 0.4232332462 \\
\hline & $\mathrm{O}$ & -1.1215495998 & -2.0434853090 & -0.6134722925 \\
\hline & $\mathrm{O}$ & -2.3257904152 & -1.3869759986 & -0.4383275471 \\
\hline \multirow[t]{20}{*}{$\mathrm{TS}_{\mathrm{R} 1-3-2}$} & $\mathrm{P}$ & -0.1918073132 & -0.5823785245 & 0.4532498368 \\
\hline & $\mathrm{O}$ & 1.1230063178 & -1.3760089695 & 0.0508750708 \\
\hline & $\mathrm{O}$ & -0.4993018711 & 0.2534824604 & -0.8703624277 \\
\hline & $\mathrm{O}$ & 0.2751879906 & 0.5048643660 & 1.5514465409 \\
\hline & $\mathrm{C}$ & -1.7398605096 & 0.9512200599 & -1.1342860223 \\
\hline & $\mathrm{H}$ & -1.8214055536 & 1.8043518039 & -0.4548547191 \\
\hline & $\mathrm{H}$ & -1.6577042305 & 1.2495681859 & -2.1791141181 \\
\hline & $\mathrm{C}$ & 0.9764415185 & 1.6602425419 & 1.2373708033 \\
\hline & $\mathrm{H}$ & 1.4997855585 & 2.0280750396 & 2.1176304508 \\
\hline & $\mathrm{H}$ & 1.9195929197 & 1.4037159403 & 0.3321099651 \\
\hline & $\mathrm{H}$ & 0.3786453111 & 2.4018866073 & 0.7010723172 \\
\hline & $\mathrm{H}$ & -2.8850252250 & -0.6040617831 & 0.6940999595 \\
\hline & $\mathrm{O}$ & -1.2521120981 & -1.4413237890 & 0.9981388256 \\
\hline & $\mathrm{O}$ & -2.8517744113 & 0.1501922297 & -1.0327419177 \\
\hline & $\mathrm{O}$ & -3.3234865057 & 0.1864461446 & 0.3104508240 \\
\hline & $\mathrm{C}$ & 2.0921056984 & -1.0366359503 & -0.9344628791 \\
\hline & $\mathrm{H}$ & 1.6041833426 & -0.6187556103 & -1.8172352961 \\
\hline & $\mathrm{H}$ & 2.6345691535 & -1.9598749072 & -1.1354997398 \\
\hline & $\mathrm{O}$ & 3.0352544891 & -0.1401202866 & -0.4357959987 \\
\hline & $\mathrm{O}$ & 2.5766864183 & 1.1546924409 & -0.6298704754 \\
\hline \multirow[t]{10}{*}{$\mathrm{TS}_{\mathrm{R} 1-3-3}$} & $\mathrm{P}$ & 0.9158755963 & -1.0044507554 & 0.4560604413 \\
\hline & $\mathrm{O}$ & -0.6616043240 & -1.3514500695 & 0.3835873682 \\
\hline & $\mathrm{O}$ & 0.9660680704 & 0.4473673916 & 1.1465984996 \\
\hline & $\mathrm{O}$ & 1.2320576185 & -0.7710853644 & -1.0874912400 \\
\hline & $\mathrm{C}$ & -0.0630525468 & 1.4029690993 & 0.9754792977 \\
\hline & $\mathrm{H}$ & -1.0002488584 & 1.0484684765 & 1.4158104026 \\
\hline & $\mathrm{H}$ & 0.3042767451 & 2.3187859703 & 1.4410276651 \\
\hline & $\mathrm{C}$ & 2.5742073718 & -0.4028597821 & -1.4540807240 \\
\hline & $\mathrm{H}$ & 3.2791057563 & -1.1454281888 & -1.0739913037 \\
\hline & $\mathrm{H}$ & 2.5935764015 & -0.3821787974 & -2.5421579873 \\
\hline
\end{tabular}




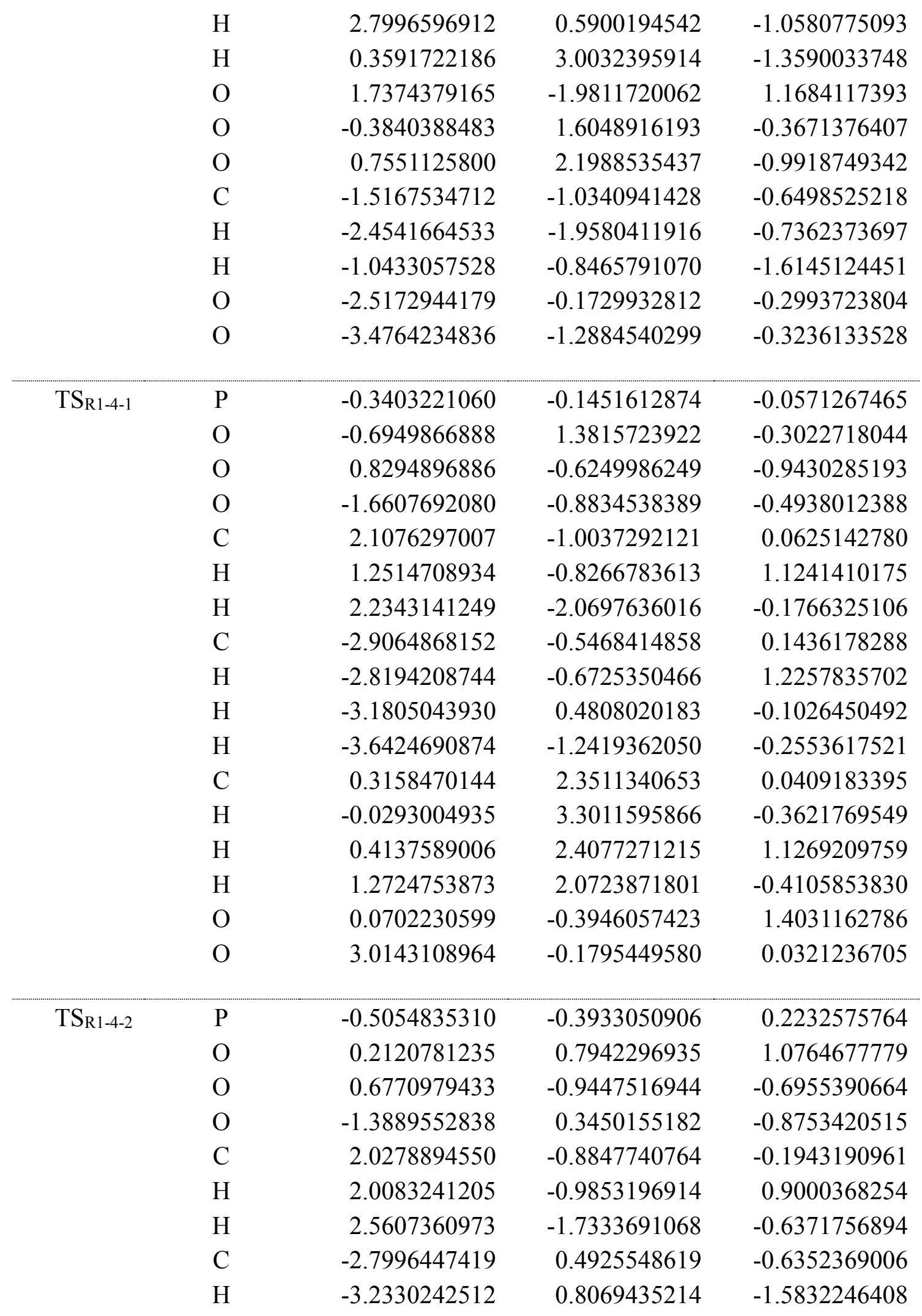




\begin{tabular}{|c|c|c|c|c|}
\hline & $\mathrm{H}$ & -3.2264194680 & -0.4579486328 & -0.3113716497 \\
\hline & $\mathrm{H}$ & -2.9723818633 & 1.2559994630 & 0.1273939508 \\
\hline & $\mathrm{C}$ & 0.9632034802 & 1.7723835790 & 0.4212524967 \\
\hline & $\mathrm{H}$ & 1.3298759020 & 2.4903542513 & 1.1530339396 \\
\hline & $\mathrm{H}$ & 1.9465688838 & 1.2216170931 & -0.0805691927 \\
\hline & $\mathrm{H}$ & 0.4439740301 & 2.2174250895 & -0.4324104795 \\
\hline & $\mathrm{O}$ & -1.2120785177 & -1.3185706337 & 1.1139286823 \\
\hline & $\mathrm{O}$ & 2.6597476211 & 0.2489368551 & -0.6148104824 \\
\hline \multirow[t]{17}{*}{$\mathrm{TS}_{\mathrm{R} 1-4-3}$} & $\mathrm{P}$ & -0.6205270995 & 0.0997553676 & 0.5131536239 \\
\hline & $\mathrm{O}$ & -0.1494254950 & 1.5613643905 & 0.8775846742 \\
\hline & $\mathrm{O}$ & 0.5164965116 & -0.4546369500 & -0.5192377765 \\
\hline & $\mathrm{O}$ & -1.8303999165 & 0.3745655198 & -0.4827278628 \\
\hline & $\mathrm{C}$ & 1.3492307195 & -1.4948258880 & -0.1577143094 \\
\hline & $\mathrm{H}$ & 1.2844651492 & -1.7944606201 & 0.8905433288 \\
\hline & $\mathrm{H}$ & 1.6776524850 & -2.3975522106 & -0.9993267942 \\
\hline & $\mathrm{C}$ & -2.5126125507 & -0.7557476217 & -1.0461168018 \\
\hline & $\mathrm{H}$ & -3.3450334789 & -0.3543022464 & -1.6212776069 \\
\hline & $\mathrm{H}$ & -1.8360223001 & -1.3077544144 & -1.7047560847 \\
\hline & $\mathrm{H}$ & -2.8826647848 & -1.4054400445 & -0.2497878705 \\
\hline & $\mathrm{C}$ & 0.1344281833 & 2.5200140977 & -0.1553171629 \\
\hline & $\mathrm{H}$ & 0.5018978196 & 3.4086232853 & 0.3545685718 \\
\hline & $\mathrm{H}$ & 0.8999483873 & 2.1281805704 & -0.8292810980 \\
\hline & $\mathrm{H}$ & -0.7792810035 & 2.7487959423 & -0.7073924466 \\
\hline & $\mathrm{O}$ & -0.8483639814 & -0.7986619017 & 1.6513643137 \\
\hline & $\mathrm{O}$ & 2.5255138449 & -1.5466097064 & -0.8155736181 \\
\hline \multirow[t]{11}{*}{$\mathrm{TS}_{\mathrm{R} 1-5-1}$} & $\mathrm{P}$ & 0.8258701578 & 0.3661928674 & -0.1064963387 \\
\hline & $\mathrm{O}$ & 1.1012391024 & 1.4742411828 & 0.9865978358 \\
\hline & $\mathrm{O}$ & -0.7731037520 & 0.1079289705 & -0.0872499617 \\
\hline & $\mathrm{O}$ & 1.3741874121 & -0.9486575970 & 0.6021266061 \\
\hline & $\mathrm{C}$ & -1.5610713707 & 0.5538685252 & -1.1907860418 \\
\hline & $\mathrm{H}$ & -1.5090188317 & 1.6517893825 & -1.3106617044 \\
\hline & $\mathrm{H}$ & -0.8921534940 & 0.2217589344 & -2.2119190646 \\
\hline & $\mathrm{C}$ & 1.2361891099 & -2.1921120240 & -0.1034444198 \\
\hline & $\mathrm{H}$ & 1.7439793547 & -2.9433535135 & 0.4988689217 \\
\hline & $\mathrm{H}$ & 0.1767617443 & -2.4449996753 & -0.2033013591 \\
\hline & $\mathrm{H}$ & 1.7070429991 & -2.1185782491 & -1.0870359315 \\
\hline
\end{tabular}




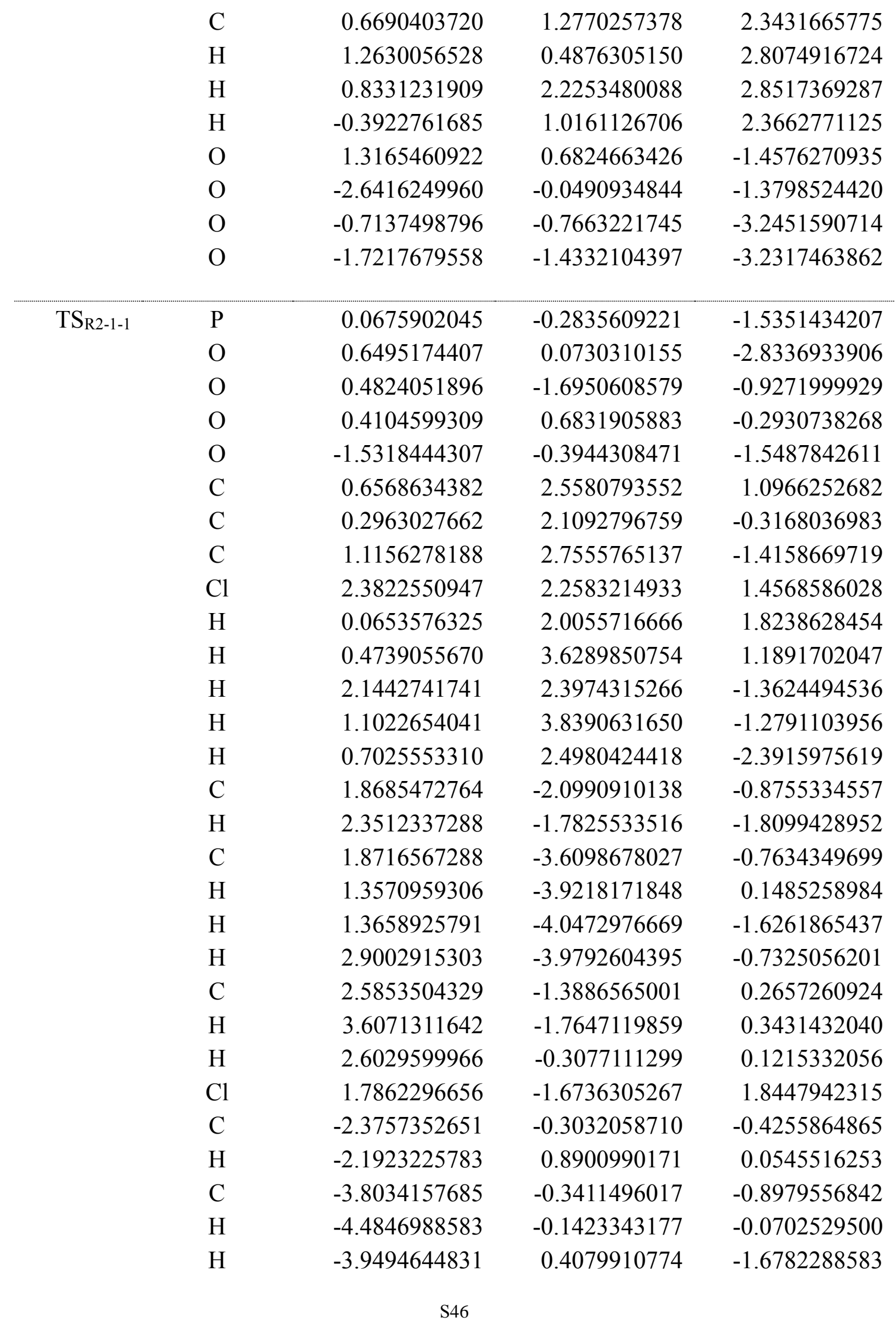




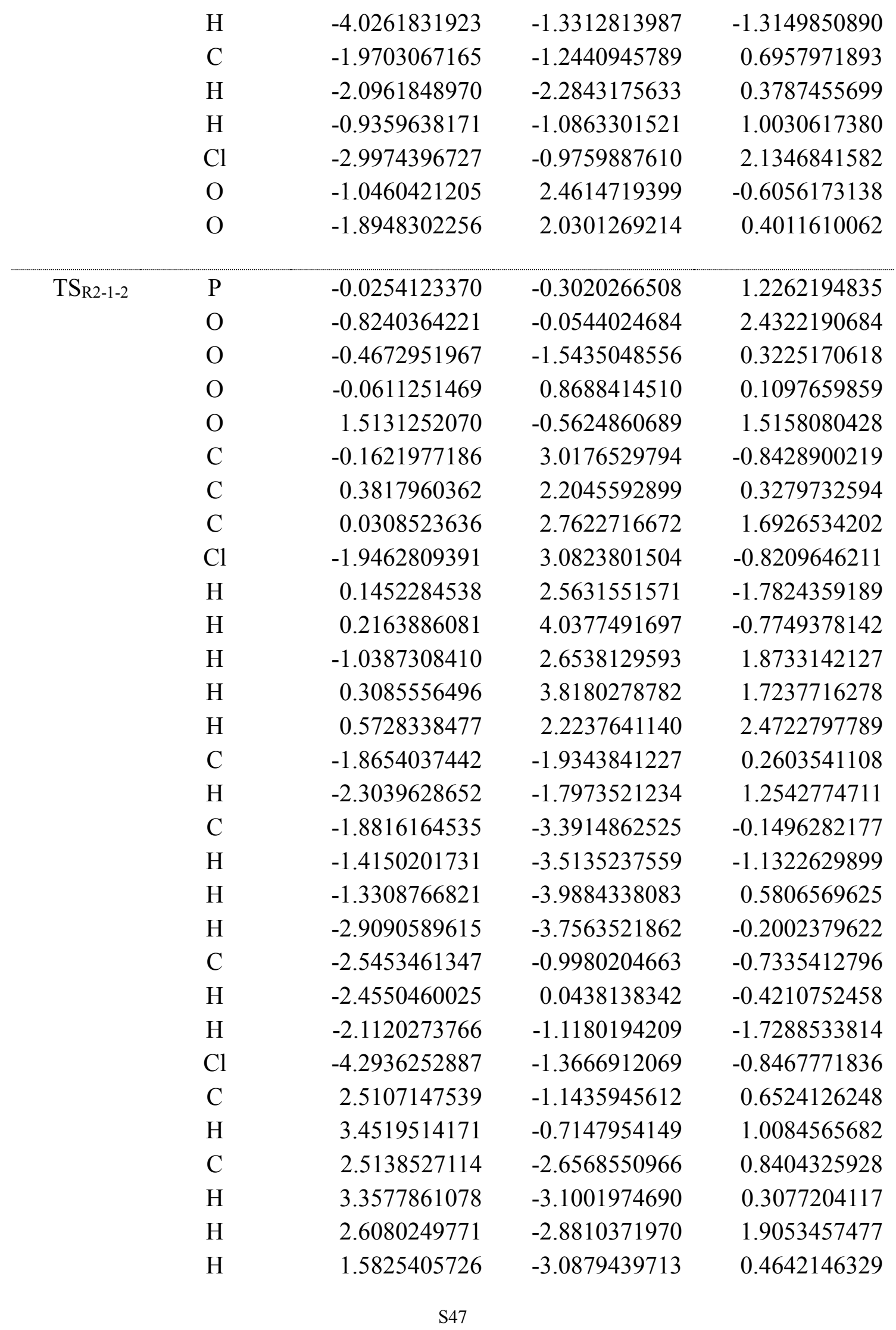




\begin{tabular}{rrrrr} 
& $\mathrm{C}$ & 2.3167390852 & -0.7351262660 & -0.7964488808 \\
$\mathrm{H}$ & 1.3766065965 & -1.0444051908 & -1.2566720633 \\
$\mathrm{H}$ & 2.2803986362 & 0.5937481533 & -0.8494743580 \\
$\mathrm{Cl}$ & 3.6773038454 & -1.1586150500 & -1.8066462049 \\
$\mathrm{O}$ & 1.8044511646 & 2.2204768125 & 0.2873536231 \\
$\mathrm{O}$ & 2.2644002498 & 1.7763039874 & -0.9378755437 \\
\hline & & & \\
$\mathrm{TS}_{\mathrm{R} 2-1-3}$ & & & \\
$\mathrm{P}$ & -0.0798653375 & -0.1053701618 & 1.5229038584 \\
$\mathrm{O}$ & 0.4949219083 & -0.2227609624 & 2.8664055532 \\
$\mathrm{O}$ & -0.7032029639 & 1.2922742375 & 1.0838217023 \\
$\mathrm{O}$ & 0.9364377831 & -0.3399931284 & 0.2865270850 \\
$\mathrm{O}$ & -1.3047614375 & -1.0845576694 & 1.2489632974 \\
$\mathrm{C}$ & 2.7679748132 & -0.9364251348 & -1.0441666722 \\
$\mathrm{C}$ & 1.9821850306 & -1.3026367602 & 0.2140408601 \\
$\mathrm{C}$ & 2.8323976399 & -1.3974017733 & 1.4663295041 \\
$\mathrm{Cl}$ & 3.5604187705 & 0.6590558662 & -0.8768198230 \\
$\mathrm{H}$ & 2.0963801477 & -0.8865510623 & -1.8991566014 \\
$\mathrm{H}$ & 3.5447751613 & -1.6816284581 & -1.2159781552 \\
$\mathrm{H}$ & 3.1751669303 & -0.4027456865 & 1.7530593788 \\
$\mathrm{H}$ & 3.6941099236 & -2.0349752142 & 1.2572576218 \\
$\mathrm{H}$ & 2.2511842312 & -1.8144717767 & 2.2883759582 \\
$\mathrm{C}$ & 0.1263246056 & 2.4180275308 & 0.7311978914 \\
$\mathrm{H}$ & 1.1490223161 & 2.2443448179 & 1.0953978814 \\
$\mathrm{C}$ & -0.4672500729 & 3.6438781036 & 1.3946064542 \\
$\mathrm{H}$ & -1.4940690962 & 3.7955112423 & 1.0536891985 \\
$\mathrm{H}$ & -0.4675906292 & 3.5133553877 & 2.4784385779 \\
$\mathrm{H}$ & 0.1235486867 & 4.5293100080 & 1.1437667893 \\
$\mathrm{C}$ & 0.2127824063 & 2.4964116102 & -0.7853611881 \\
$\mathrm{H}$ & 0.7818149990 & 3.3788172721 & -1.0832281901 \\
$\mathrm{H}$ & 0.6892587870 & 1.6022253557 & -1.1860643519 \\
$\mathrm{Cl}$ & -1.4094430161 & 2.6173649300 & -1.5450386479 \\
$\mathrm{C}$ & -1.9154527304 & -1.1117261147 & -0.0578598048 \\
$\mathrm{H}$ & -1.4666042412 & -0.3488047974 & -0.7066908737 \\
$\mathrm{C}$ & -1.7133986456 & -2.4825726052 & -0.6306897138 \\
$\mathrm{H}$ & -2.1291059025 & -2.6350998092 & -1.6263448235 \\
$\mathrm{H}$ & -0.3972640744 & -2.6081126430 & -0.8646480121 \\
$\mathrm{H}$ & -1.8968285416 & -3.2991364765 & 0.0699274974 \\
$\mathrm{C}$ & -3.3766642202 & -0.7339432298 & 0.1837877080 \\
& & & \\
& & $\mathrm{~S} 48$ & & \\
& & & & \\
& & & \\
& & & \\
& & &
\end{tabular}




\begin{tabular}{rrrrr} 
& $\mathrm{H}$ & -3.8596394935 & -1.4618345806 & 0.8374203590 \\
$\mathrm{H}$ & -3.4155003412 & 0.2603934662 & 0.6309250599 \\
$\mathrm{Cl}$ & -4.2836301667 & -0.6790302242 & -1.3559616723 \\
$\mathrm{O}$ & 1.4293328858 & -2.6002712927 & 0.0592886959 \\
$\mathrm{O}$ & 0.7429638844 & -2.7080752668 & -1.1432874022 \\
& & & \\
\hline $\mathrm{TS}_{\mathrm{R} 2-1-4}$ & & & \\
$\mathrm{P}$ & 0.3721306090 & 0.0164211872 & 1.4535839579 \\
$\mathrm{O}$ & 1.2333718264 & 0.0706005677 & 2.6391537573 \\
$\mathrm{O}$ & -0.5441793224 & 1.2663980820 & 1.1134053080 \\
$\mathrm{O}$ & 1.1843910594 & -0.0901271704 & 0.0439317250 \\
$\mathrm{O}$ & -0.6634813408 & -1.1829463803 & 1.4440897354 \\
$\mathrm{C}$ & 3.3273809214 & -0.4923101799 & -1.0178010662 \\
$\mathrm{C}$ & 2.0916119665 & -1.0886888543 & -0.3068720283 \\
$\mathrm{C}$ & 2.5141775513 & -2.0242540174 & 0.8116170106 \\
$\mathrm{Cl}$ & 3.0657758288 & 0.9689991671 & -1.9120050910 \\
$\mathrm{H}$ & 3.2921756369 & -1.5336009196 & -1.8538761833 \\
$\mathrm{H}$ & 4.2557817465 & -0.4579428947 & -0.4506828701 \\
$\mathrm{H}$ & 3.0139525146 & -1.4740628267 & 1.6102639729 \\
$\mathrm{H}$ & 3.1807218537 & -2.7813720072 & 0.3935628533 \\
$\mathrm{H}$ & 1.6313128572 & -2.5205835799 & 1.2229509772 \\
$\mathrm{C}$ & -0.0376597414 & 2.4732817466 & 0.5034560646 \\
$\mathrm{H}$ & 1.0575990028 & 2.4888666941 & 0.5868166308 \\
$\mathrm{C}$ & -0.6395065488 & 3.6452364038 & 1.2506716110 \\
$\mathrm{H}$ & -1.7296445816 & 3.6083329104 & 1.1923941595 \\
$\mathrm{H}$ & -0.3372449009 & 3.6097684544 & 2.2990316197 \\
$\mathrm{H}$ & -0.2933194343 & 4.5856949647 & 0.8128468232 \\
$\mathrm{C}$ & -0.3660940526 & 2.4246689410 & -0.9809112754 \\
$\mathrm{H}$ & -0.0398164715 & 3.3470602651 & -1.4656140268 \\
$\mathrm{H}$ & 0.1213609647 & 1.5697572717 & -1.4499776546 \\
$\mathrm{Cl}$ & -2.1268582931 & 2.2522723855 & -1.2706407170 \\
$\mathrm{C}$ & -1.5836594704 & -1.3678608948 & 0.3290700795 \\
$\mathrm{H}$ & -1.2338145966 & -0.7835945457 & -0.5311272451 \\
$\mathrm{C}$ & -1.5890285002 & -2.8465764984 & 0.0063847430 \\
$\mathrm{H}$ & -2.2938021436 & -3.0509356950 & -0.8021793409 \\
$\mathrm{H}$ & -0.5920498133 & -3.1594943734 & -0.3123403960 \\
$\mathrm{H}$ & -1.8835622253 & -3.4199012194 & 0.8905764608 \\
$\mathrm{C}$ & -2.9201439123 & -0.8141780135 & 0.8059937433 \\
$\mathrm{H}$ & -3.2839109044 & -1.3840870890 & 1.6630403928 \\
& & & \\
& & $\mathrm{~S} 49$ & & \\
& & & & \\
& & & \\
& & &
\end{tabular}




\begin{tabular}{rrrrr} 
& $\mathrm{H}$ & -2.8181288550 & 0.2402897956 & 1.0637658989 \\
$\mathrm{Cl}$ & -4.1494649874 & -0.9290225344 & -0.4905931784 \\
$\mathrm{O}$ & 1.4306000478 & -1.7970585611 & -1.365655856 \\
$\mathrm{O}$ & 2.4681500089 & -2.4340047922 & -2.0373590564 \\
\hline $\mathrm{TS}_{\mathrm{R} 2-1-5}$ & & & \\
\hline $\mathrm{P}$ & 0.2231248152 & -0.2063160931 & 1.4617849118 \\
$\mathrm{O}$ & 1.0325544215 & -0.2423682211 & 2.6867057037 \\
$\mathrm{O}$ & -0.5604642653 & 1.1373148583 & 1.1196124098 \\
$\mathrm{O}$ & 1.0812903164 & -0.3423782842 & 0.0841374285 \\
$\mathrm{O}$ & -0.9363304521 & -1.2753623972 & 1.3889033449 \\
$\mathrm{C}$ & 2.7674608649 & -0.7507510309 & -1.4965144832 \\
$\mathrm{C}$ & 2.1679573025 & -1.2062628842 & -0.1739320139 \\
$\mathrm{C}$ & 3.1447017988 & -1.3435076168 & 0.9888782820 \\
$\mathrm{Cl}$ & 3.4862642320 & 0.8739385918 & -1.3178179448 \\
$\mathrm{H}$ & 1.9871735549 & -0.7003261358 & -2.2570274698 \\
$\mathrm{H}$ & 3.5543877088 & -1.4388526611 & -1.8029507006 \\
$\mathrm{H}$ & 4.0982241913 & -1.7865812078 & 0.7020296943 \\
$\mathrm{H}$ & 2.4360815045 & -2.3620148306 & 1.4451276915 \\
$\mathrm{H}$ & 3.1797101820 & -0.5536285015 & 1.7320339959 \\
$\mathrm{C}$ & 0.1200217032 & 2.3357373425 & 0.7005237797 \\
$\mathrm{H}$ & 1.1909539048 & 2.2463139258 & 0.9321798316 \\
$\mathrm{C}$ & -0.4874336493 & 3.4943055779 & 1.4649139050 \\
$\mathrm{H}$ & -1.5583758965 & 3.5591397304 & 1.2587463117 \\
$\mathrm{H}$ & -0.3394557597 & 3.3482566342 & 2.5365844096 \\
$\mathrm{H}$ & -0.0117622131 & 4.4324699097 & 1.1654669963 \\
$\mathrm{C}$ & 0.0047929990 & 2.4471938190 & -0.8123725882 \\
$\mathrm{H}$ & 0.4498304059 & 3.3833747357 & -1.1542201283 \\
$\mathrm{H}$ & 0.5045163052 & 1.6072717465 & -1.2936219002 \\
$\mathrm{Cl}$ & -1.7036540845 & 2.4247735608 & -1.3565823260 \\
$\mathrm{C}$ & -1.7498738678 & -1.4236032708 & 0.1952620093 \\
$\mathrm{H}$ & -1.2624261247 & -0.9110892968 & -0.6428226760 \\
$\mathrm{C}$ & -1.8623373873 & -2.9088917769 & -0.0751730626 \\
$\mathrm{H}$ & -2.4734951279 & -3.0860235887 & -0.9627752265 \\
$\mathrm{H}$ & -0.8664372398 & -3.3291765075 & -0.2323883840 \\
$\mathrm{H}$ & -2.3231291489 & -3.4088257627 & 0.7818594821 \\
$\mathrm{C}$ & -3.0682525315 & -0.7261226821 & 0.5048711053 \\
$\mathrm{H}$ & -3.5857907903 & -1.2334389108 & 1.3209876940 \\
$\mathrm{H}$ & -2.8889246504 & 0.3194830879 & 0.7571751063 \\
& & & \\
& & 550 & & \\
& & & & \\
& & & \\
& & &
\end{tabular}




\begin{tabular}{|c|c|c|c|c|}
\hline & \multirow{3}{*}{$\begin{array}{l}\mathrm{Cl} \\
\mathrm{O} \\
\mathrm{O}\end{array}$} & \multirow{3}{*}{$\begin{array}{r}-4.1440527875 \\
1.7226381501 \\
1.5186345455\end{array}$} & \multirow{3}{*}{$\begin{array}{l}-0.7531395948 \\
-2.5284843055 \\
-3.0217503293\end{array}$} & \multirow{3}{*}{$\begin{array}{r}-0.9273770692 \\
-0.3860904278 \\
0.9195560177\end{array}$} \\
\hline & & & & \\
\hline & & & & \\
\hline \multirow[t]{34}{*}{$\mathrm{TS}_{\mathrm{R} 2-1-6}$} & $\mathrm{P}$ & 0.2418098668 & -0.4031709585 & 0.9529473748 \\
\hline & $\mathrm{O}$ & 1.1443180682 & -0.3278068652 & 2.1121897435 \\
\hline & $\mathrm{O}$ & -0.6513084166 & 0.7929728828 & 0.4087248063 \\
\hline & $\mathrm{O}$ & 1.6616733848 & 0.4489845722 & -0.3505365816 \\
\hline & $\mathrm{O}$ & -1.0070277676 & -1.3552273807 & 1.3158139097 \\
\hline & $\mathrm{C}$ & 3.2181036122 & -0.2102824640 & -2.0421705650 \\
\hline & $\mathrm{C}$ & 2.6870666607 & -0.2239841211 & -0.6180068700 \\
\hline & $\mathrm{C}$ & 3.7202185086 & -0.5577244353 & 0.4289680481 \\
\hline & $\mathrm{Cl}$ & 1.9463627689 & 0.0400876209 & -3.2561484586 \\
\hline & $\mathrm{H}$ & 3.7262117161 & -1.1501412925 & -2.2629298199 \\
\hline & $\mathrm{H}$ & 3.9365622318 & 0.6098165933 & -2.1264433769 \\
\hline & $\mathrm{H}$ & 4.3615927424 & 0.3228986322 & 0.5467768835 \\
\hline & $\mathrm{H}$ & 4.3399023593 & -1.4021346596 & 0.1199277064 \\
\hline & $\mathrm{H}$ & 3.2299687780 & -0.7566832197 & 1.3820803926 \\
\hline & $\mathrm{C}$ & -0.3020319690 & 2.1674345920 & 0.1573553436 \\
\hline & $\mathrm{H}$ & 0.7470195567 & 2.3262547222 & 0.4205502560 \\
\hline & $\mathrm{C}$ & -1.2028787730 & 3.0208426197 & 1.0307603184 \\
\hline & $\mathrm{H}$ & -2.2536836034 & 2.8482874062 & 0.7863782510 \\
\hline & $\mathrm{H}$ & -1.0346790767 & 2.7829993962 & 2.0834476699 \\
\hline & $\mathrm{H}$ & -0.9764898513 & 4.0789840095 & 0.8696240777 \\
\hline & $\mathrm{C}$ & -0.4464312400 & 2.4135926375 & -1.3335729638 \\
\hline & $\mathrm{H}$ & -0.2321879987 & 3.4587866902 & -1.5638956813 \\
\hline & $\mathrm{H}$ & 0.2258624095 & 1.7591466461 & -1.8867656080 \\
\hline & $\mathrm{Cl}$ & -2.1132585671 & 2.0683722919 & -1.9056091939 \\
\hline & $\mathrm{C}$ & -2.1095953194 & -1.5684001668 & 0.4072427880 \\
\hline & $\mathrm{H}$ & -1.8491950707 & -1.2041785682 & -0.5947698870 \\
\hline & $\mathrm{C}$ & -2.3903066656 & -3.0575318506 & 0.3837753629 \\
\hline & $\mathrm{H}$ & -3.2298445328 & -3.2763330132 & -0.2792417469 \\
\hline & $\mathrm{H}$ & -1.5062856874 & -3.5928705191 & 0.0310843361 \\
\hline & $\mathrm{H}$ & -2.6321166195 & -3.4048306405 & 1.3927878916 \\
\hline & $\mathrm{C}$ & -3.2599464379 & -0.7267193420 & 0.9474050684 \\
\hline & $\mathrm{H}$ & -3.5490799320 & -1.0735602198 & 1.9413466896 \\
\hline & $\mathrm{H}$ & -2.9714254796 & 0.3230496326 & 0.9799290639 \\
\hline & $\mathrm{Cl}$ & -4.6993108894 & -0.8515583060 & -0.1153587301 \\
\hline
\end{tabular}




\begin{tabular}{clrrr} 
& $\mathrm{O}$ & 1.8136140521 & -1.9353074943 & -0.7558171375 \\
$\mathrm{O}$ & 0.6549713014 & -1.7922085381 & -0.2485090513 \\
\hline $\mathrm{TS}_{\mathrm{R} 2-3-1}$ & & & \\
\hline $\mathrm{P}$ & -0.1430567999 & -0.7037732906 & -0.7238502461 \\
$\mathrm{O}$ & -0.4422428949 & -1.7046224331 & -1.7682902510 \\
$\mathrm{O}$ & -0.9454094411 & -0.9319417945 & 0.6544410304 \\
$\mathrm{O}$ & 1.3608233407 & -0.6110320710 & -0.2294203723 \\
$\mathrm{O}$ & -0.4803063356 & 0.7664977413 & -1.2231223125 \\
$\mathrm{C}$ & 3.5366697429 & -1.5400948722 & -0.0700075209 \\
$\mathrm{C}$ & 2.0924143080 & -1.7006587353 & 0.3927496105 \\
$\mathrm{C}$ & 1.8958239766 & -1.6510610926 & 1.8947450006 \\
$\mathrm{Cl}$ & 4.2418328488 & 0.0033472902 & 0.4923780735 \\
$\mathrm{H}$ & 3.5835994535 & -1.5534962369 & -1.1558291782 \\
$\mathrm{H}$ & 4.1302590643 & -2.3558796792 & 0.3437623688 \\
$\mathrm{H}$ & 2.5444435273 & -2.3896401310 & 2.3702348746 \\
$\mathrm{H}$ & 0.8542505325 & -1.8722779004 & 2.1321702942 \\
$\mathrm{H}$ & 2.1483462297 & -0.6551428167 & 2.2609893260 \\
$\mathrm{C}$ & -2.3205804819 & -0.6877998986 & 0.8199945675 \\
$\mathrm{H}$ & -2.3864176314 & 0.6134030921 & 0.8536596658 \\
$\mathrm{C}$ & -2.7328541403 & -1.1590088177 & 2.1873947219 \\
$\mathrm{H}$ & -2.6649620982 & -2.2530460646 & 2.2367174684 \\
$\mathrm{H}$ & -2.0652308634 & -0.7295362493 & 2.9372773456 \\
$\mathrm{H}$ & -3.7581563222 & -0.8570195528 & 2.4026806782 \\
$\mathrm{C}$ & -3.1552113547 & -1.1293841744 & -0.3694251478 \\
$\mathrm{H}$ & -2.8001312937 & -0.6845609469 & -1.3000588565 \\
$\mathrm{H}$ & -3.1313072248 & -2.2190627406 & -0.4682104113 \\
$\mathrm{Cl}$ & -4.8549829744 & -0.6239170526 & -0.1531580177 \\
$\mathrm{C}$ & -0.3408771436 & 2.0263110817 & -0.5179266308 \\
$\mathrm{H}$ & 0.9874628528 & -2.8729948303 & -1.7850340684 \\
$\mathrm{C}$ & -1.1472272587 & 3.0218059236 & -1.3226861258 \\
$\mathrm{H}$ & -2.1561724702 & 2.6393782980 & -1.4715053724 \\
$\mathrm{H}$ & -1.1907646162 & 3.9718364182 & -0.7898450386 \\
$\mathrm{H}$ & -0.6654556684 & 3.1687309555 & -2.2917285110 \\
$\mathrm{C}$ & 1.1512492737 & 2.3425788405 & -0.4074842551 \\
$\mathrm{H}$ & 1.6330477847 & 1.6693988096 & 0.2996250830 \\
$\mathrm{H}$ & 1.6078717845 & 2.2269087131 & -1.3909244972 \\
$\mathrm{Cl}$ & 1.4415506573 & 4.0127232579 & 0.1480213714 \\
$\mathrm{O}$ & 1.5760910184 & -2.9352027765 & -0.0077139063 \\
& & & \\
& & $\mathrm{~S} 52$ & & \\
& & & & \\
& & & \\
& & & \\
& & &
\end{tabular}




\begin{tabular}{|c|c|c|c|c|}
\hline & $\mathrm{O}$ & 1.8393481163 & -3.1640561574 & -1.3890077046 \\
\hline & $\mathrm{O}$ & -0.7822023643 & 1.8811914778 & 0.8119303089 \\
\hline & $\mathrm{O}$ & -2.1621481337 & 1.8143814158 & 0.8702776355 \\
\hline \multirow[t]{34}{*}{$\mathrm{TS}_{\mathrm{R} 2-3-2}$} & $\mathrm{P}$ & 0.0422023595 & -0.6068110489 & 0.6516337238 \\
\hline & $\mathrm{O}$ & 0.3279235998 & -1.7067114572 & 1.5995560538 \\
\hline & $\mathrm{O}$ & 0.7274271610 & -0.7450333361 & -0.7797788186 \\
\hline & $\mathrm{O}$ & -1.4849522841 & -0.3417780748 & 0.2960521839 \\
\hline & $\mathrm{O}$ & 0.4982098510 & 0.7943527265 & 1.2556912143 \\
\hline & $\mathrm{C}$ & -3.7294218106 & -1.0989383338 & 0.2444421383 \\
\hline & $\mathrm{C}$ & -2.3402272941 & -1.3177221961 & -0.3466769480 \\
\hline & $\mathrm{C}$ & -2.2585150297 & -1.1583281888 & -1.8516872267 \\
\hline & $\mathrm{Cl}$ & -4.3628946379 & 0.5259623308 & -0.1495541171 \\
\hline & $\mathrm{H}$ & -3.6896682758 & -1.1901871835 & 1.3267685095 \\
\hline & $\mathrm{H}$ & -4.4116574833 & -1.8400338563 & -0.1729886504 \\
\hline & $\mathrm{H}$ & -2.9770214005 & -1.8281368730 & -2.3286129801 \\
\hline & $\mathrm{H}$ & -1.2498496023 & -1.4027904454 & -2.1880845764 \\
\hline & $\mathrm{H}$ & -2.4928461383 & -0.1272913826 & -2.1190778521 \\
\hline & $\mathrm{C}$ & 2.0250650617 & -1.3568523466 & -0.9821177513 \\
\hline & $\mathrm{H}$ & 2.3608996240 & -0.9488658932 & -1.9396033965 \\
\hline & $\mathrm{C}$ & 1.8635392218 & -2.8691944987 & -1.0603877030 \\
\hline & $\mathrm{H}$ & 1.5146294545 & -3.2603715887 & -0.1015440669 \\
\hline & $\mathrm{H}$ & 1.1285920197 & -3.1130659499 & -1.8317464764 \\
\hline & $\mathrm{H}$ & 2.8169985312 & -3.3348623160 & -1.3200328537 \\
\hline & $\mathrm{C}$ & 2.9980956627 & -0.9182687864 & 0.0982701198 \\
\hline & $\mathrm{H}$ & 2.9492989241 & 0.4179652342 & 0.1451782042 \\
\hline & $\mathrm{H}$ & 2.7524573355 & -1.2397156653 & 1.1129829782 \\
\hline & $\mathrm{Cl}$ & 4.6582705188 & -1.2801556443 & -0.2930674245 \\
\hline & $\mathrm{C}$ & 0.6892447827 & 2.0500913959 & 0.5761600962 \\
\hline & $\mathrm{H}$ & -1.1840319830 & -2.7429083717 & 1.6413187552 \\
\hline & $\mathrm{C}$ & 1.0794833002 & 3.0327970270 & 1.6573311158 \\
\hline & $\mathrm{H}$ & 1.9313017194 & 2.6356269801 & 2.2088427899 \\
\hline & $\mathrm{H}$ & 1.3464080497 & 3.9876724748 & 1.2039422465 \\
\hline & $\mathrm{H}$ & 0.2378914794 & 3.1731697267 & 2.3390518337 \\
\hline & $\mathrm{C}$ & -0.5789300639 & 2.4021310980 & -0.2022184715 \\
\hline & $\mathrm{H}$ & -0.6968665936 & 1.7194686265 & -1.0446433728 \\
\hline & $\mathrm{H}$ & -1.4424447432 & 2.3191563867 & 0.4578409975 \\
\hline & $\mathrm{Cl}$ & -0.5285491199 & 4.0600158609 & -0.8581967258 \\
\hline
\end{tabular}




\begin{tabular}{rrrrr} 
& $\mathrm{O}$ & -1.8899074599 & -2.6179881562 & -0.0917806966 \\
$\mathrm{O}$ & -2.0747001123 & -2.9550686256 & 1.2801352828 \\
$\mathrm{O}$ & 1.6836639735 & 1.9040183289 & -0.4189780343 \\
$\mathrm{O}$ & 2.9037414024 & 1.6012410224 & 0.1492318993 \\
\hline $\mathrm{TS}_{\mathrm{R} 2-3-3}$ & & & \\
\hline $\mathrm{P}$ & -0.3140202525 & 0.3511131405 & -0.7494303175 \\
$\mathrm{O}$ & -0.9042149325 & 0.0417055144 & -2.0692718323 \\
$\mathrm{O}$ & -1.3943582100 & 0.6130826783 & 0.3982752860 \\
$\mathrm{O}$ & 0.6306851337 & -0.7300490174 & -0.0825142292 \\
$\mathrm{O}$ & 0.5897087571 & 1.6593232746 & -0.8338887668 \\
$\mathrm{C}$ & 1.5362596753 & -2.9039994957 & 0.0637778628 \\
$\mathrm{C}$ & 0.2461706378 & -2.1009663636 & 0.1919143387 \\
$\mathrm{C}$ & -0.4391197242 & -2.1910415820 & 1.5409973095 \\
$\mathrm{Cl}$ & 2.7161158484 & -2.4626891211 & 1.3332882065 \\
$\mathrm{H}$ & 1.9980443958 & -2.7091308642 & -0.9014997446 \\
$\mathrm{H}$ & 1.3042656499 & -3.9638714088 & 0.1718567969 \\
$\mathrm{H}$ & -0.5889926650 & -3.2410585981 & 1.8015024458 \\
$\mathrm{H}$ & -1.4051572191 & -1.6843653911 & 1.4963498096 \\
$\mathrm{H}$ & 0.1852746715 & -1.7158566413 & 2.2981660663 \\
$\mathrm{C}$ & -2.4646502297 & 1.5311607935 & 0.1174769510 \\
$\mathrm{H}$ & -2.2790340907 & 2.0390239150 & -0.8397509010 \\
$\mathrm{C}$ & -2.4841250179 & 2.5272607278 & 1.2383945648 \\
$\mathrm{H}$ & -2.5437504949 & 2.0919220040 & 2.2361668020 \\
$\mathrm{H}$ & -1.2371896582 & 3.0423238287 & 1.2558004868 \\
$\mathrm{H}$ & -3.1197658334 & 3.3994275335 & 1.0837089543 \\
$\mathrm{C}$ & -3.7470407433 & 0.7299317476 & -0.0716951236 \\
$\mathrm{H}$ & -4.5759656515 & 1.3925221728 & -0.3253583080 \\
$\mathrm{H}$ & -3.5936260128 & -0.0017663215 & -0.8669133683 \\
$\mathrm{Cl}$ & -4.2039984572 & -0.1672350790 & 1.4088374254 \\
$\mathrm{C}$ & 1.4308581871 & 2.2598895302 & 0.1537231477 \\
$\mathrm{H}$ & -0.4642333928 & -1.7399337330 & -2.4002799028 \\
$\mathrm{C}$ & 1.9268440896 & 3.5488083921 & -0.4662175184 \\
$\mathrm{H}$ & 1.0807832584 & 4.1526763347 & -0.7914823860 \\
$\mathrm{H}$ & 2.5081276715 & 4.1046423909 & 0.2729442321 \\
$\mathrm{H}$ & 2.5554751018 & 3.3095455331 & -1.3251927532 \\
$\mathrm{C}$ & 2.5632170904 & 1.3304459041 & 0.6098657026 \\
$\mathrm{H}$ & 3.2485260605 & 1.9047733303 & 1.2329955506 \\
$\mathrm{H}$ & 2.1692331535 & 0.4831492228 & 1.1692978477 \\
& & & \\
& & $\mathrm{~S} 54$ & & \\
& & & & \\
& & & \\
& & & \\
& & &
\end{tabular}




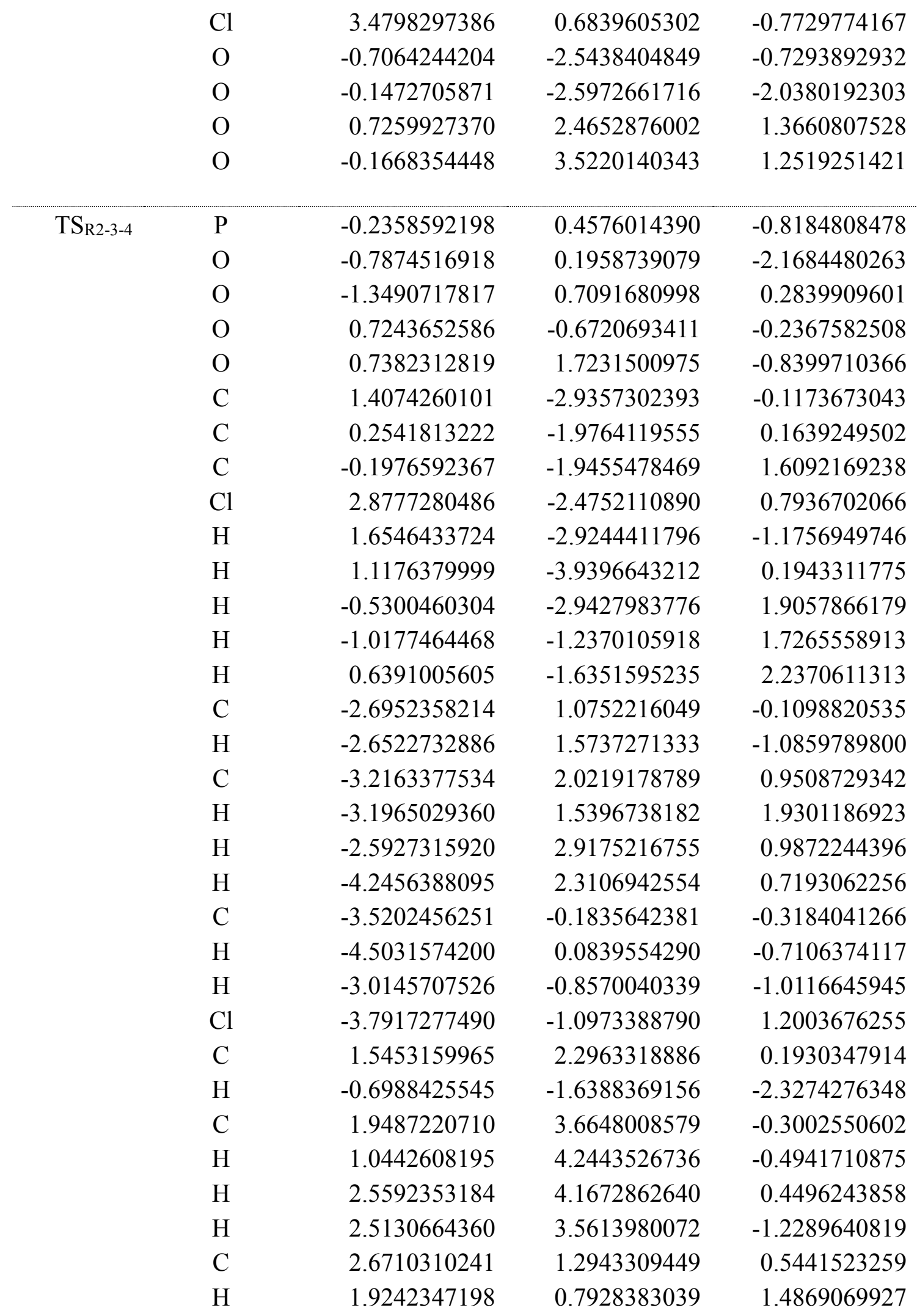




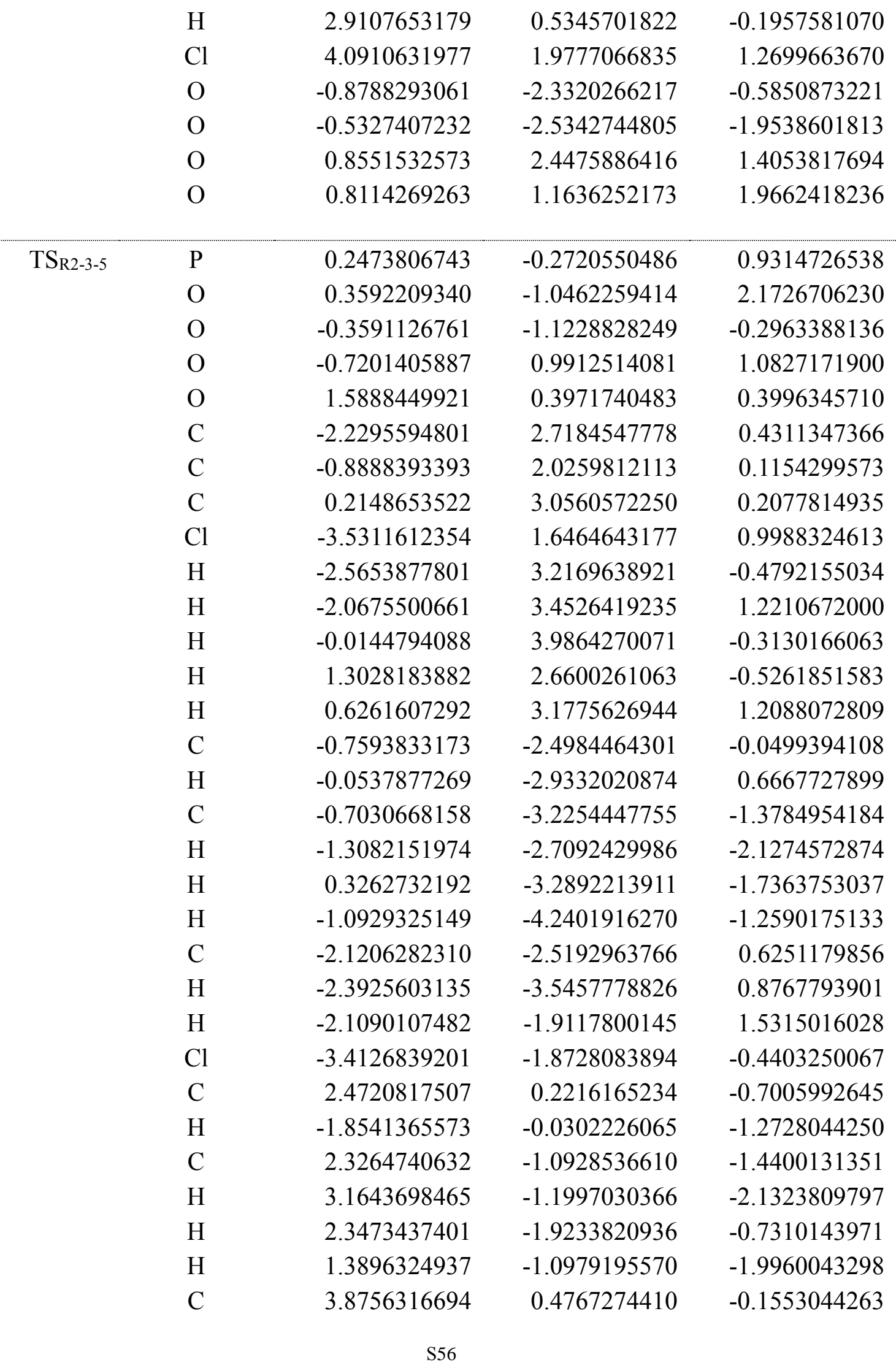




\begin{tabular}{|c|c|c|c|c|}
\hline & $\mathrm{H}$ & 4.5792285252 & 0.5052236898 & -0.9879815325 \\
\hline & $\mathrm{H}$ & 3.8952359088 & 1.4190657251 & 0.3877800250 \\
\hline & $\mathrm{Cl}$ & 4.3817696621 & -0.8183219687 & 0.9625022627 \\
\hline & $\mathrm{O}$ & -0.8075605600 & 1.5363350121 & -1.1940574856 \\
\hline & $\mathrm{O}$ & -2.0169830226 & 0.8902349660 & -1.5543528999 \\
\hline & $\mathrm{O}$ & 2.1506501982 & 1.2110033166 & -1.6610734364 \\
\hline & $\mathrm{O}$ & 2.2843163525 & 2.4743384255 & -1.1115768897 \\
\hline \multirow[t]{30}{*}{$\mathrm{TS}_{\mathrm{R} 2-3-6}$} & $\mathrm{P}$ & -0.2433044905 & 0.2951742082 & -0.7374173489 \\
\hline & $\mathrm{O}$ & -0.4116394096 & -0.1430506287 & -2.1305842243 \\
\hline & $\mathrm{O}$ & -1.6049289171 & 0.6214977715 & 0.0260050408 \\
\hline & $\mathrm{O}$ & 0.5309121899 & -0.7152401473 & 0.2162901555 \\
\hline & $\mathrm{O}$ & 0.6495352563 & 1.6192545024 & -0.6176229691 \\
\hline & $\mathrm{C}$ & 1.4286107147 & -1.6054289588 & 2.1598156217 \\
\hline & $\mathrm{C}$ & 0.1760851804 & -1.5756819930 & 1.2967214637 \\
\hline & $\mathrm{C}$ & -0.9978735053 & -1.1265532492 & 2.1562878141 \\
\hline & $\mathrm{Cl}$ & 2.9473978986 & -2.0564024900 & 1.4505928735 \\
\hline & $\mathrm{H}$ & 1.2881604550 & -2.0862705828 & 3.1268507027 \\
\hline & $\mathrm{H}$ & 1.5365475649 & -0.2836448335 & 2.4065358616 \\
\hline & $\mathrm{H}$ & -1.0485307647 & -1.7831834343 & 3.0275246140 \\
\hline & $\mathrm{H}$ & -1.9261004471 & -1.2080502782 & 1.5918933770 \\
\hline & $\mathrm{H}$ & -0.8587978689 & -0.0932912085 & 2.4783837279 \\
\hline & $\mathrm{C}$ & -2.7383777387 & 1.1231572982 & -0.7202753504 \\
\hline & $\mathrm{H}$ & -2.3727324019 & 1.7754901829 & -1.5247757344 \\
\hline & $\mathrm{C}$ & -3.5797026870 & 1.9153387578 & 0.2593915304 \\
\hline & $\mathrm{H}$ & -3.9040237574 & 1.2735745518 & 1.0817746948 \\
\hline & $\mathrm{H}$ & -2.9965725292 & 2.7440656113 & 0.6659949913 \\
\hline & $\mathrm{H}$ & -4.4632692435 & 2.3162054356 & -0.2450367073 \\
\hline & $\mathrm{C}$ & -3.4662525217 & -0.0258031024 & -1.3999992022 \\
\hline & $\mathrm{H}$ & -4.3247081259 & 0.3590476321 & -1.9534970528 \\
\hline & $\mathrm{H}$ & -2.7925700090 & -0.5533311453 & -2.0747242032 \\
\hline & $\mathrm{Cl}$ & -4.0890087410 & -1.2256980189 & -0.2193682802 \\
\hline & $\mathrm{C}$ & 1.4319480702 & 2.0337874781 & 0.5001957403 \\
\hline & $\mathrm{H}$ & 0.5366969420 & -2.8842529340 & -0.9352967593 \\
\hline & $\mathrm{C}$ & 1.3481728237 & 3.5448060374 & 0.5506289689 \\
\hline & $\mathrm{H}$ & 0.3089315216 & 3.8299998318 & 0.7263601755 \\
\hline & $\mathrm{H}$ & 1.9669568458 & 3.9131406229 & 1.3724859726 \\
\hline & $\mathrm{H}$ & 1.6908100537 & 3.9720673703 & -0.3921769252 \\
\hline
\end{tabular}




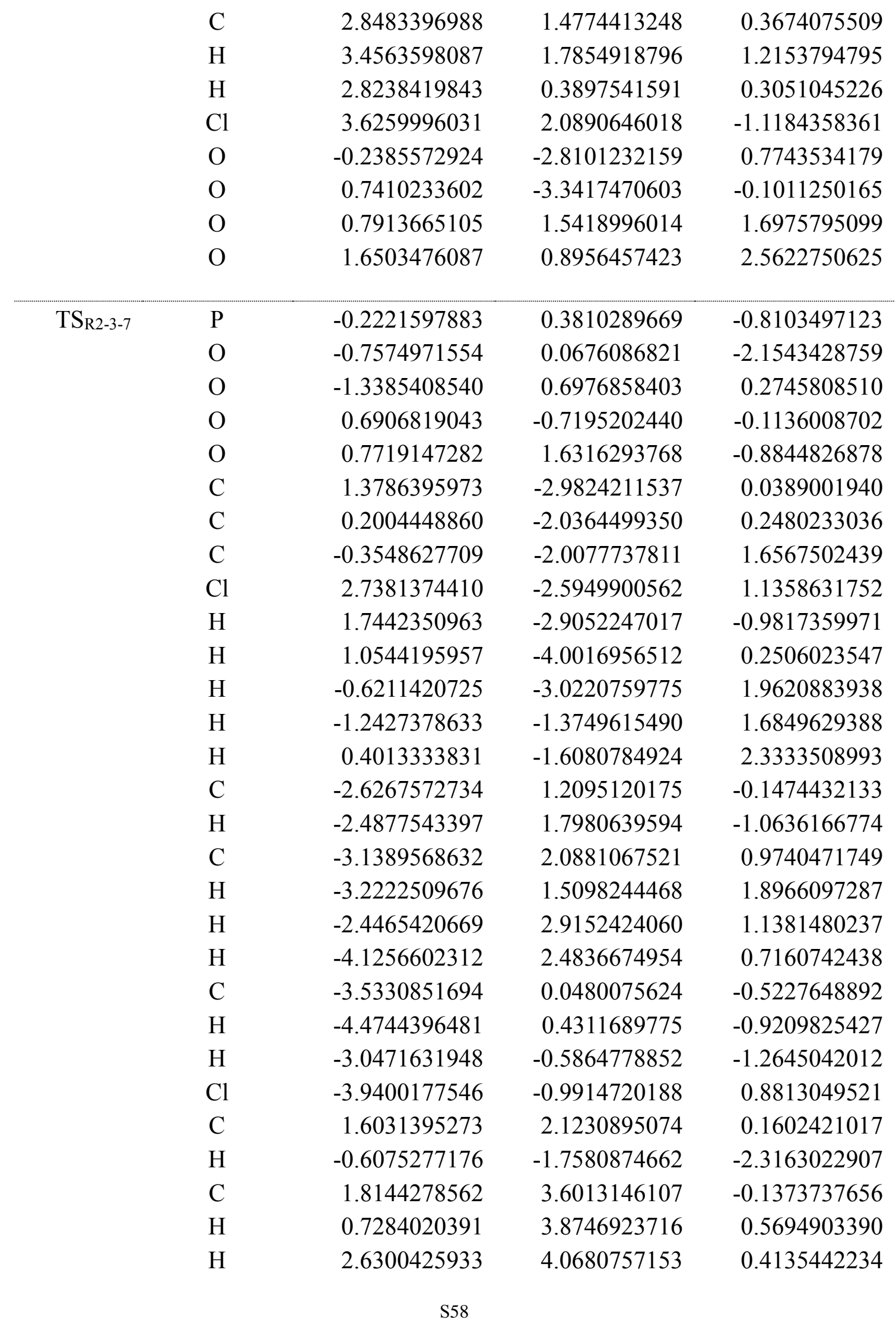




\begin{tabular}{|c|c|c|c|c|}
\hline & $\mathrm{H}$ & 1.7002850588 & 3.9135051040 & -1.1713803066 \\
\hline & $\mathrm{C}$ & 2.8702640365 & 1.2941535287 & 0.3339918607 \\
\hline & $\mathrm{H}$ & 3.4727637505 & 1.7386941898 & 1.1259395179 \\
\hline & $\mathrm{H}$ & 2.6296938440 & 0.2590698718 & 0.5735498739 \\
\hline & $\mathrm{Cl}$ & 3.8237307671 & 1.3175951266 & -1.1719088620 \\
\hline & $\mathrm{O}$ & -0.8750353390 & -2.3999923020 & -0.5720985569 \\
\hline & $\mathrm{O}$ & -0.4311134277 & -2.6350689097 & -1.9064137036 \\
\hline & $\mathrm{O}$ & 0.9510422057 & 2.1127244376 & 1.4067733833 \\
\hline & $\mathrm{O}$ & 0.0359646575 & 3.1819932771 & 1.3266968854 \\
\hline \multirow[t]{28}{*}{$\mathrm{TS}_{\mathrm{R} 2-5-1}$} & $\mathrm{P}$ & 0.4247508876 & 0.2333284066 & 0.2319149413 \\
\hline & $\mathrm{O}$ & 0.4765716397 & 0.7656075565 & 1.6217207238 \\
\hline & $\mathrm{O}$ & -0.3028364345 & 1.1544861297 & -0.8406107769 \\
\hline & $\mathrm{O}$ & 1.8159002498 & 0.0142994073 & -0.4866561007 \\
\hline & $\mathrm{O}$ & -0.2540785084 & -1.2074001428 & 0.0965756378 \\
\hline & $\mathrm{C}$ & 2.5575600233 & -2.3156888929 & -0.4122539550 \\
\hline & $\mathrm{C}$ & 2.8505235952 & -0.8687380345 & 0.0000559523 \\
\hline & $\mathrm{C}$ & 4.1516645119 & -0.3187243603 & -0.5365487314 \\
\hline & $\mathrm{Cl}$ & 2.2741872161 & -2.4655447825 & -2.1646584453 \\
\hline & $\mathrm{H}$ & 1.6656553287 & -2.6729880830 & 0.1014181993 \\
\hline & $\mathrm{H}$ & 3.4154323080 & -2.9348172430 & -0.1513481715 \\
\hline & $\mathrm{H}$ & 4.9767396778 & -0.9308400939 & -0.1651522259 \\
\hline & $\mathrm{H}$ & 4.2802056090 & 0.7080653956 & -0.1965156268 \\
\hline & $\mathrm{H}$ & 4.1338123136 & -0.34655556887 & -1.6271421372 \\
\hline & $\mathrm{H}$ & -0.6421313797 & -0.2787500402 & 2.7189762357 \\
\hline & $\mathrm{C}$ & -1.5952453348 & -1.5377770171 & 0.5315122241 \\
\hline & $\mathrm{H}$ & 2.3191939615 & 0.6795494075 & 2.1478725341 \\
\hline & C & -1.5478646807 & -3.0006577435 & 0.9291703495 \\
\hline & $\mathrm{H}$ & -2.5187793032 & -3.3002243218 & 1.3250862959 \\
\hline & $\mathrm{H}$ & -1.3040287918 & -3.6074514113 & 0.0538356772 \\
\hline & $\mathrm{H}$ & -0.7855160162 & -3.1316250828 & 1.6970925296 \\
\hline & $\mathrm{C}$ & -2.5157122885 & -1.2330523256 & -0.6458891471 \\
\hline & $\mathrm{H}$ & -2.7420665768 & 0.0942649310 & -0.6632822896 \\
\hline & $\mathrm{H}$ & -2.0537084718 & -1.4399939662 & -1.6111603010 \\
\hline & $\mathrm{Cl}$ & -4.1168650046 & -1.9023612075 & -0.5388728915 \\
\hline & $\mathrm{O}$ & 2.8011610507 & -0.9586168647 & 1.3974771932 \\
\hline & $\mathrm{O}$ & 3.1957587081 & 0.2746980795 & 1.9812972120 \\
\hline & $\mathrm{O}$ & -2.0273831105 & -0.6816644158 & 1.5431678316 \\
\hline
\end{tabular}




\begin{tabular}{rrrrr}
$\mathrm{O}$ & -1.3371375674 & -0.9697706408 & 2.7508057675 \\
$\mathrm{C}$ & -1.0780102361 & 2.3351602893 & -0.6017745823 \\
$\mathrm{C}$ & -0.4448258680 & 3.2952772337 & 0.4096185454 \\
$\mathrm{H}$ & -1.0118480881 & 4.2258512736 & 0.4005094875 \\
$\mathrm{H}$ & -0.4469889518 & 2.8526922676 & 1.4037396526 \\
$\mathrm{C}$ & -1.3159834044 & 2.9530696539 & -1.9616937356 \\
$\mathrm{H}$ & -0.3674508803 & 3.3164431513 & -2.3604739042 \\
$\mathrm{H}$ & -1.7362134184 & 2.2102600348 & -2.6384068416 \\
$\mathrm{H}$ & -2.0158779783 & 3.7852121928 & -1.8577804868 \\
$\mathrm{Cl}$ & 1.2454024453 & 3.6675490705 & -0.0184236839 \\
$\mathrm{O}$ & -2.2718054477 & 1.9566370808 & 0.0574893761 \\
$\mathrm{O}$ & -3.0766137840 & 1.2212036768 & -0.7838323323 \\
& & & \\
\hline $\mathrm{P}$ & -0.1824146562 & -0.6208428955 & 0.8002308175 \\
$\mathrm{O}$ & -0.1199633055 & -0.5574725829 & 2.2773824620 \\
$\mathrm{O}$ & -0.2712703031 & 0.7890738213 & 0.0473372605 \\
$\mathrm{O}$ & 0.9801632809 & -1.4061007456 & 0.0603520187 \\
$\mathrm{O}$ & -1.3976763532 & -1.4527168155 & 0.1980642533 \\
$\mathrm{C}$ & 2.8144359368 & -0.0038239553 & -0.5120054000 \\
$\mathrm{C}$ & 2.4085239544 & -1.2812492543 & 0.2155240055 \\
$\mathrm{C}$ & 2.8488493514 & -1.3560456220 & 1.6629967242 \\
$\mathrm{Cl}$ & 4.5261336123 & 0.2011294845 & -0.6947947685 \\
$\mathrm{H}$ & 2.4317569511 & 0.9846021356 & 0.2854051275 \\
$\mathrm{H}$ & 2.2729343190 & 0.1889149099 & -1.4381444121 \\
$\mathrm{H}$ & 3.9391758277 & -1.3419092023 & 1.7025947666 \\
$\mathrm{H}$ & 2.4758958936 & -2.2834007684 & 2.1005591920 \\
$\mathrm{H}$ & 2.4461189196 & -0.5104437293 & 2.2239259274 \\
$\mathrm{H}$ & -1.9115188949 & -0.5493647095 & 2.7908100162 \\
$\mathrm{C}$ & -2.7572171940 & -0.9799528585 & 0.2838303303 \\
$\mathrm{H}$ & 1.7438179991 & -2.8011522818 & -1.7953136462 \\
$\mathrm{C}$ & -3.6324857357 & -2.1828474061 & 0.5450533968 \\
$\mathrm{H}$ & -3.3170071730 & -2.6781560736 & 1.4617110181 \\
$\mathrm{H}$ & -4.6691882791 & -1.8544425158 & 0.6505956993 \\
$\mathrm{H}$ & -3.5499494038 & -2.8749866351 & -0.2937550524 \\
$\mathrm{C}$ & -3.1021459826 & -0.2111892866 & -0.9952424017 \\
$\mathrm{H}$ & -4.1393814251 & 0.1195339326 & -0.9421632599 \\
$\mathrm{H}$ & -2.4335710231 & 0.6418175500 & -1.1087081949 \\
$\mathrm{Cl}$ & -2.9147070537 & -1.2195228153 & -2.4519649327 \\
& & & \\
\hline & & $\mathrm{S} 60$ & & \\
\hline
\end{tabular}




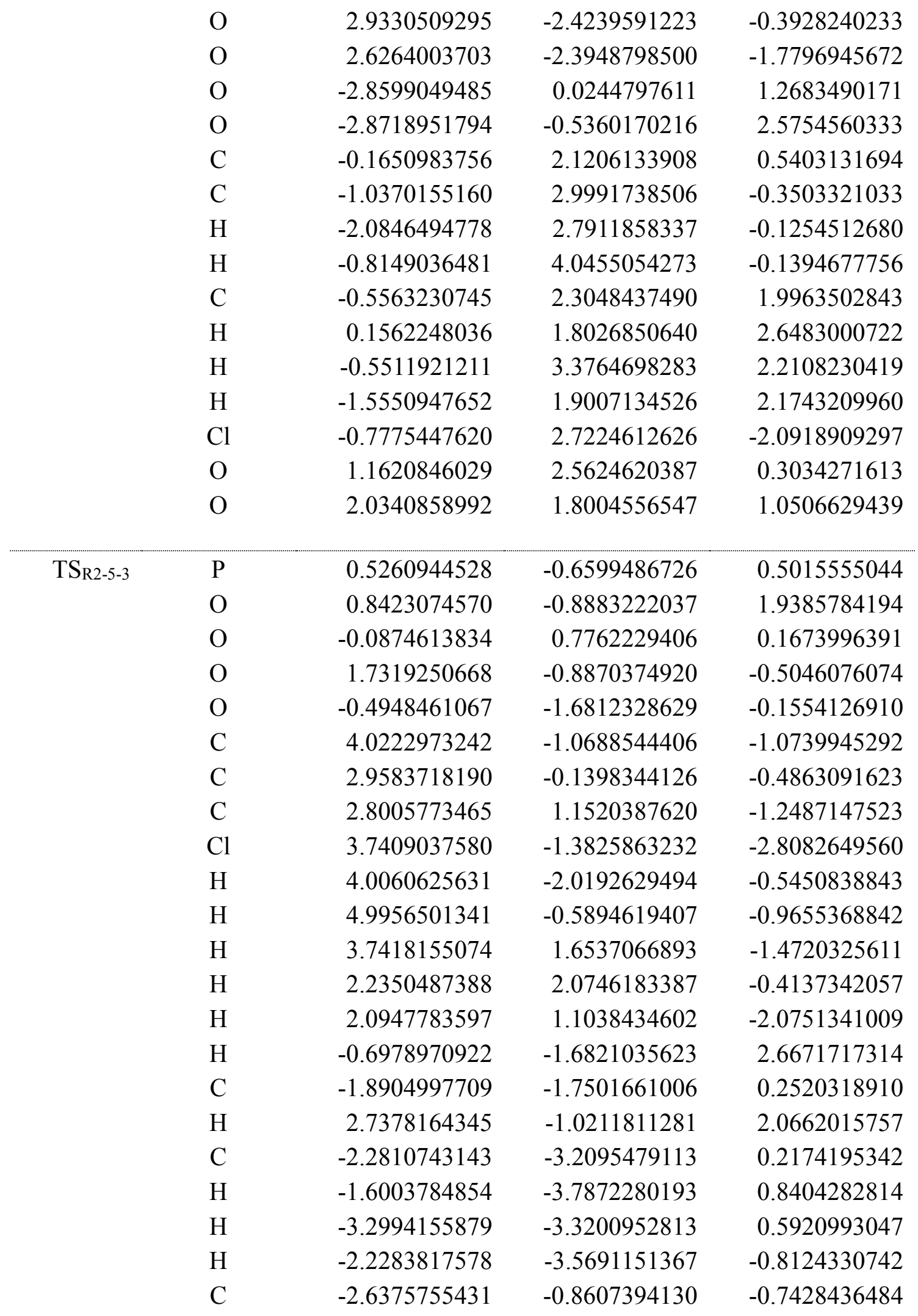




$\begin{array}{lrrr}\mathrm{H} & -2.3142885061 & 0.1746989865 & -0.6335996218 \\ \mathrm{H} & -2.4099918229 & -1.2079139230 & -1.7505678295 \\ \mathrm{Cl} & -4.4042795634 & -0.9261581023 & -0.5148242024 \\ \mathrm{O} & 3.3096326038 & 0.2393341690 & 0.8147619943 \\ \mathrm{O} & 3.5917349105 & -0.9166491652 & 1.5954818039 \\ \mathrm{O} & -2.0583478607 & -1.1357121961 & 1.4978605427 \\ \mathrm{O} & -1.6224044231 & -1.9893179660 & 2.5471591507 \\ \mathrm{C} & -0.1202929127 & 1.9358717887 & 1.0094794802 \\ \mathrm{C} & -0.9068364023 & 3.0052342104 & 0.2384934965 \\ \mathrm{H} & -1.9701233608 & 2.8645083013 & 0.4365780270 \\ \mathrm{H} & -0.5920290358 & 3.9838212978 & 0.6022157706 \\ \mathrm{C} & -0.7705843556 & 1.6784636923 & 2.3579231768 \\ \mathrm{H} & -0.1131424302 & 1.0653162800 & 2.9746543268 \\ \mathrm{H} & -0.9416482465 & 2.6366749093 & 2.8544539773 \\ \mathrm{H} & -1.7196065014 & 1.1579758319 & 2.2155251348 \\ \mathrm{Cl} & -0.7186119392 & 2.9878658954 & -1.5354732441 \\ \mathrm{O} & 1.1956411789 & 2.3296166598 & 1.3144688769 \\ \mathrm{O} & 1.7998465875 & 2.9333349897 & 0.2261389652\end{array}$

\section{$\mathrm{TS}_{\mathrm{R} 2-5-4}$}

\section{$\mathrm{P}$}

$\mathrm{O}$

$\mathrm{O}$

$\mathrm{O}$

$\mathrm{O}$

C

C

C

$\mathrm{Cl}$

$\mathrm{H}$

$\mathrm{H}$

$\mathrm{H}$

$\mathrm{H}$

$\mathrm{H}$

$\mathrm{H}$

$\mathrm{C}$

$\mathrm{H}$

C

$\mathrm{H}$

$\mathrm{P}$
$\mathrm{O}$
$\mathrm{O}$
$\mathrm{O}$
$\mathrm{O}$
$\mathrm{C}$
$\mathrm{C}$
$\mathrm{C}$
$\mathrm{Cl}$
$\mathrm{H}$
$\mathrm{H}$
$\mathrm{H}$
$\mathrm{H}$
$\mathrm{H}$
$\mathrm{H}$
$\mathrm{C}$
$\mathrm{H}$
$\mathrm{C}$
$\mathrm{H}$

$-0.3875704787$

$-0.4248070687$

0.0920714459

$-1.7640855905$

0.5055922429

$-4.1206490072$

$-2.9266953385$

$-3.0392159340$

$-4.5019802052$

$-3.9113278659$

$-4.9923378047$

$-3.9463971496$

$-2.1652686845$

$-3.0768910401$

0.9006778484

1.9492052082

$-2.0807287884$

2.5145332535

1.8643853689
$-0.2753493154$

0.3650388262

0.6666086560

$-0.9096307644$

$-1.5778302044$

$-0.7966009937$

$-0.0931229172$

0.1176112678

$-2.3377752964$

$-1.0185051246$

$-0.1455814578$

0.6821908674

0.6658277087

$-0.8514561305$

$-3.9379921496$

$-1.6612685298$

0.9984847869

$-1.1217929159$

$-1.2200661986$
$-0.4722686211$

$-1.8058866375$

0.7231059394

0.0112703923

$-0.3288391936$

$-0.3648397027$

0.2747643340

1.7707305539

0.4529164136

$-1.4075730278$

$-0.2898308132$

1.9967035765

2.1241769404

2.2679410065

0.4717737570

$-0.3366633987$

$-2.0214349096$

0.9548056411

1.8209726294 


$\begin{array}{lrrr}\mathrm{H} & 3.5571795252 & -1.3901900320 & 1.1143672811 \\ \mathrm{H} & 2.6142842243 & 0.2392729732 & 0.7838989825 \\ \mathrm{C} & 2.4711795309 & -1.0051714641 & -1.6168483019 \\ \mathrm{H} & 2.1067579683 & -1.5724408812 & -2.4738131532 \\ \mathrm{H} & 2.1265004134 & 0.0288776446 & -1.6784490460 \\ \mathrm{Cl} & 4.2522336152 & -0.9827087937 & -1.6600711293 \\ \mathrm{O} & -2.7625088314 & 1.1949859131 & -0.2710011651 \\ \mathrm{O} & -2.9856219480 & 1.1956175037 & -1.6796676713 \\ \mathrm{O} & 2.2196464248 & -3.0245259007 & -0.4964670947 \\ \mathrm{O} & 1.8218175504 & -3.7116636137 & 0.6810786871 \\ \mathrm{C} & 0.4995911512 & 2.0394866997 & 0.8034943060 \\ \mathrm{C} & 0.2919963958 & 2.8799304654 & -0.4649631178 \\ \mathrm{H} & -0.6912896078 & 2.6545465487 & -0.8791869991 \\ \mathrm{H} & 0.3148437093 & 3.9213927879 & -0.1432606864 \\ \mathrm{C} & -0.3062144093 & 2.6674900867 & 1.9300782575 \\ \mathrm{H} & -0.2660906477 & 2.0132042694 & 2.8028224536 \\ \mathrm{H} & 0.1190123555 & 3.6385474156 & 2.1903334447 \\ \mathrm{H} & -1.3432434640 & 2.7969310120 & 1.6093864413 \\ \mathrm{Cl} & 1.4876756571 & 2.7601616553 & -1.7788473041 \\ \mathrm{O} & 1.8238572154 & 2.0292264579 & 1.2900511490 \\ \mathrm{O} & 2.6650374292 & 1.3314626274 & 0.4353999165\end{array}$

$\begin{array}{rrrrr}\text { TS }_{\text {R2-55 }} & \mathrm{P} & -0.2721598888 & 0.2830746066 & 0.0229451051 \\ & \mathrm{O} & -0.4396876502 & 0.9605954212 & -1.2959382278 \\ \mathrm{O} & 0.7500140405 & 1.0341227974 & 0.9908881075 \\ \mathrm{O} & -1.5831525731 & 0.1898069771 & 0.9041134852 \\ \mathrm{O} & 0.2407613372 & -1.2095085601 & 0.0011314535 \\ \mathrm{C} & -2.9055915284 & -1.6194554726 & -0.0877834302 \\ \mathrm{C} & -2.9167083298 & -0.1978200119 & 0.4747284875 \\ \mathrm{C} & -3.7866602799 & -0.0033283866 & 1.6994386936 \\ \mathrm{Cl} & -2.4866433566 & -2.8222415600 & 1.1618953579 \\ \mathrm{H} & -2.1831924262 & -1.7228015526 & -0.8953839607 \\ \mathrm{H} & -3.9018844735 & -1.8480839788 & -0.4673798849 \\ \mathrm{H} & -4.7961386787 & -0.3607313102 & 1.4882413192 \\ \mathrm{H} & -3.8222432138 & 1.0621466528 & 1.9359250579 \\ \mathrm{H} & -3.3679674940 & -0.5519268255 & 2.5425998143 \\ \mathrm{H} & 0.4413208797 & -0.1142329372 & -2.6113425888 \\ \mathrm{C} & 1.4692714068 & -1.6877239175 & -0.6332693660\end{array}$




$\begin{array}{lrrr}\mathrm{H} & -2.1802626913 & 0.8051844506 & -1.8891951148 \\ \mathrm{C} & 1.1693924188 & -3.0950835820 & -1.0949833138 \\ \mathrm{H} & 0.3192169359 & -3.0822003423 & -1.7762152568 \\ \mathrm{H} & 2.0423342835 & -3.4963714801 & -1.6115011907 \\ \mathrm{H} & 0.9352796635 & -3.7177376922 & -0.2287518708 \\ \mathrm{C} & 2.5466833870 & -1.5650739971 & 0.4444904520 \\ \mathrm{H} & 2.7315631523 & -0.5120872201 & 0.6615217423 \\ \mathrm{H} & 2.1959687595 & -2.0744385325 & 1.3421027288 \\ \mathrm{Cl} & 4.0880780077 & -2.3090582278 & -0.0508893291 \\ \mathrm{O} & -3.4129757677 & 0.7117073877 & -0.4661494529 \\ \mathrm{O} & -3.0486699790 & 0.3605173352 & -1.7967475258 \\ \mathrm{O} & 1.8644526655 & -0.8170160502 & -1.6431530303 \\ \mathrm{O} & 0.9813554972 & -0.9185140952 & -2.7522916131 \\ \mathrm{C} & 0.8232339242 & 2.4461380860 & 1.0502893109 \\ \mathrm{C} & 1.5572188515 & 3.0917937607 & -0.1456583706 \\ \mathrm{H} & 0.9423783511 & 3.5822991791 & -0.8975459962 \\ \mathrm{H} & 2.0903685690 & 3.9485839794 & 0.7173441264 \\ \mathrm{C} & -0.5246784179 & 3.0985628377 & 1.2968923169 \\ \mathrm{H} & -0.9730952004 & 2.6494350125 & 2.1851623213 \\ \mathrm{H} & -0.3706435535 & 4.1656812538 & 1.4682613204 \\ \mathrm{H} & -1.1904169898 & 2.9611768822 & 0.4406890217 \\ \mathrm{Cl} & 2.8266151987 & 2.1570341425 & -0.8506273261 \\ \mathrm{O} & 1.7228841858 & 2.6555119239 & 2.1302879545 \\ \mathrm{O} & 2.1164660971 & 3.9804884062 & 1.9636350124\end{array}$

\footnotetext{
$\mathrm{TS}_{\mathrm{R} 2-5-6}$
}

$\begin{array}{lr}\mathrm{P} & -0.4096930567 \\ \mathrm{O} & -0.5916147042 \\ \mathrm{O} & 0.6226365943 \\ \mathrm{O} & -1.7085098023 \\ \mathrm{O} & 0.0843683066 \\ \mathrm{C} & -2.8057015982 \\ \mathrm{C} & -2.9758888969 \\ \mathrm{C} & -3.8968855971 \\ \mathrm{Cl} & -2.1490073745 \\ \mathrm{H} & -2.1284361249 \\ \mathrm{H} & -3.7877245178 \\ \mathrm{H} & -4.8605189369 \\ \mathrm{H} & -4.0408882007\end{array}$

$\begin{array}{rr}0.3813295498 & 0.0382645132 \\ 1.1942055984 & -1.2029920392 \\ 1.0197045712 & 1.0625204086 \\ 0.2173519178 & 0.9268454835 \\ -1.1073474460 & -0.1773965210 \\ -1.8984684193 & 0.3603044800 \\ -0.3973803820 & 0.5991497548 \\ -0.0503448563 & 1.7504112418 \\ -2.7273711175 & 1.7979237589 \\ -2.0910718969 & -0.4671080242 \\ -2.3191819133 & 0.1408430843 \\ -0.5429700441 & 1.6093519766 \\ 1.0313446388 & 1.7745607531\end{array}$




\begin{tabular}{|c|c|c|c|}
\hline $\mathrm{H}$ & -3.4467971783 & -0.3799004738 & 2.6868147594 \\
\hline $\mathrm{H}$ & 0.4996620978 & 0.4289015590 & -2.5249892921 \\
\hline $\mathrm{C}$ & 1.3794394892 & -1.4368996475 & -0.7773694053 \\
\hline $\mathrm{H}$ & -2.2686028506 & 0.5076274486 & -1.8660997213 \\
\hline $\mathrm{C}$ & 1.1601845773 & -2.6918721787 & -1.5893148325 \\
\hline $\mathrm{H}$ & 0.3759293214 & -2.5241890882 & -2.3263341199 \\
\hline $\mathrm{H}$ & 2.0872361670 & -2.9554131576 & -2.1002254609 \\
\hline $\mathrm{H}$ & 0.8694752469 & -3.5030974091 & -0.9176423409 \\
\hline $\mathrm{C}$ & 2.3263355342 & -1.5904526974 & 0.4121692618 \\
\hline $\mathrm{H}$ & 2.4401861791 & -0.6324859813 & 0.9199782797 \\
\hline $\mathrm{H}$ & 1.9018360360 & -2.3296605711 & 1.0917863666 \\
\hline $\mathrm{Cl}$ & 3.9445772537 & -2.1474353501 & -0.0830227844 \\
\hline $\mathrm{O}$ & -3.5515042273 & 0.2154940875 & -0.5198306082 \\
\hline $\mathrm{O}$ & -2.9092234659 & -0.2162102471 & -1.7156789820 \\
\hline $\mathrm{O}$ & 1.8575332681 & -0.3460090144 & -1.5041846931 \\
\hline $\mathrm{O}$ & 1.1781856580 & -0.2417244424 & -2.7498497123 \\
\hline $\mathrm{C}$ & 0.9616753748 & 2.4146074939 & 1.1129993513 \\
\hline $\mathrm{C}$ & 2.0267376277 & 2.7334661370 & 0.0772414350 \\
\hline $\mathrm{H}$ & 1.6491613616 & 2.4676719362 & -0.9119418869 \\
\hline $\mathrm{H}$ & 2.2649728787 & 3.7962273668 & 0.1228945168 \\
\hline $\mathrm{C}$ & -0.2768332277 & 3.3071063977 & 1.0556049817 \\
\hline $\mathrm{H}$ & -1.1416613534 & 2.9764391243 & 0.4871218332 \\
\hline $\mathrm{H}$ & -0.4207837205 & 3.1286946609 & 2.3722588673 \\
\hline $\mathrm{H}$ & -0.0459744752 & 4.3680770069 & 0.9661191658 \\
\hline $\mathrm{Cl}$ & 3.5329991882 & 1.8266512275 & 0.3662459939 \\
\hline $\mathrm{O}$ & 1.4771303258 & 2.6649610402 & 2.3876829987 \\
\hline $\mathrm{O}$ & 0.3456850527 & 2.6961811417 & 3.2222249186 \\
\hline $\mathrm{P}$ & 0.6286117870 & -0.0420441723 & -0.1929280054 \\
\hline $\mathrm{O}$ & 0.8325275779 & 0.0377200444 & -1.6563083629 \\
\hline $\mathrm{O}$ & 1.9314994146 & -0.0049772926 & 0.7162879615 \\
\hline $\mathrm{O}$ & -0.2997651678 & 1.0565315327 & 0.4773980892 \\
\hline $\mathrm{O}$ & -0.0835242220 & -1.3814406974 & 0.3250580527 \\
\hline $\mathrm{C}$ & -1.8224584639 & 2.8146362227 & 0.1644115601 \\
\hline $\mathrm{C}$ & -0.3383050167 & 2.4713162075 & 0.1737383302 \\
\hline $\mathrm{C}$ & 0.5044660277 & 3.2087725691 & 1.1966425385 \\
\hline $\mathrm{Cl}$ & -2.5922085602 & 2.8010509448 & 1.7299337378 \\
\hline $\mathrm{H}$ & -2.4144402459 & 1.8432890646 & -0.5192629572 \\
\hline
\end{tabular}




\begin{tabular}{|c|c|c|c|c|}
\hline & $\mathrm{H}$ & -2.0583121526 & 3.7186102553 & -0.3972930756 \\
\hline & $\mathrm{H}$ & 0.3845776549 & 4.2865182219 & 1.0700878781 \\
\hline & $\mathrm{H}$ & 1.5512181156 & 2.9348667692 & 1.0404426164 \\
\hline & $\mathrm{H}$ & 0.1954107360 & 2.9160095701 & 2.1999624242 \\
\hline & $\mathrm{H}$ & -2.7374267669 & -3.0691487252 & 1.8974880031 \\
\hline & $\mathrm{C}$ & -1.2411670031 & -2.0253314860 & -0.2422558545 \\
\hline & $\mathrm{H}$ & -0.1474048494 & 1.4329602180 & -2.3051898166 \\
\hline & $\mathrm{C}$ & -0.9640067069 & -2.5542004149 & -1.6337250615 \\
\hline & $\mathrm{H}$ & -1.8542564625 & -3.0584502786 & -2.0131605764 \\
\hline & $\mathrm{H}$ & -0.6861475405 & -1.7329431045 & -2.2941417135 \\
\hline & $\mathrm{H}$ & -0.1340957067 & -3.2613518660 & -1.5690919579 \\
\hline & $\mathrm{H}$ & 1.2780603750 & -2.6650408791 & 1.0869104792 \\
\hline & $\mathrm{O}$ & 0.2323271080 & 2.7698472257 & -1.0566854521 \\
\hline & $\mathrm{O}$ & -0.5975374310 & 2.2841638163 & -2.1060704949 \\
\hline & $\mathrm{O}$ & -1.4442922806 & -3.1494333837 & 0.5511968282 \\
\hline & $\mathrm{O}$ & -1.8247901795 & -2.7372695272 & 1.8620315064 \\
\hline & $\mathrm{C}$ & 3.1046276632 & -0.7918602429 & 0.4100885068 \\
\hline & $\mathrm{C}$ & 3.7956672985 & -0.2736807045 & -0.8557735428 \\
\hline & $\mathrm{H}$ & 4.7338293982 & -0.8119892168 & -0.9892764714 \\
\hline & $\mathrm{H}$ & 3.1457909424 & -0.4095795153 & -1.7196348255 \\
\hline & $\mathrm{Cl}$ & 4.1671072169 & 1.4662398988 & -0.7270832036 \\
\hline & $\mathrm{O}$ & 2.7145155373 & -2.0856053074 & 0.0268643677 \\
\hline & $\mathrm{O}$ & 2.2409491510 & -2.8087368296 & 1.1544965474 \\
\hline & $\mathrm{C}$ & -2.4204545109 & -1.0284314784 & -0.1514182770 \\
\hline & $\mathrm{H}$ & -2.4367811537 & -0.5174598843 & 0.8121662794 \\
\hline & $\mathrm{Cl}$ & -3.9854102719 & -1.8988860240 & -0.3189285825 \\
\hline & $\mathrm{O}$ & -2.2502233763 & -0.1156558684 & -1.1931830759 \\
\hline & $\mathrm{O}$ & -3.0846906878 & 0.9654311921 & -0.9961699188 \\
\hline & $\mathrm{C}$ & 3.9699698393 & -0.7528181502 & 1.6485189602 \\
\hline & $\mathrm{H}$ & 4.8373902699 & -1.3985542226 & 1.4935208779 \\
\hline & $\mathrm{H}$ & 4.3015969271 & 0.2716387330 & 1.8237389561 \\
\hline & $\mathrm{H}$ & 3.4036657161 & -1.1096282139 & 2.5073557251 \\
\hline $\mathrm{TS}_{\mathrm{R} 2-7-2}$ & $\mathrm{P}$ & -0.0383199683 & -0.3366216187 & 0.2719306379 \\
\hline & $\mathrm{O}$ & -0.0145882335 & 0.1646767286 & 1.6758516137 \\
\hline & $\mathrm{O}$ & 1.3126007358 & -0.2246657373 & -0.5350464761 \\
\hline & $\mathrm{O}$ & -0.4176714715 & -1.8740746431 & 0.1267153178 \\
\hline & $\mathrm{O}$ & -1.0711869754 & 0.4032288452 & -0.6855125363 \\
\hline
\end{tabular}




\begin{tabular}{|c|c|c|c|}
\hline $\mathrm{C}$ & -2.6352291514 & -2.3138412246 & -0.7749328423 \\
\hline $\mathrm{C}$ & -1.7276817762 & -2.3814834610 & 0.4576312627 \\
\hline $\mathrm{C}$ & -1.5332597638 & -3.7702753810 & 1.0170142137 \\
\hline $\mathrm{Cl}$ & -1.9319083463 & -3.1814122031 & -2.1610514077 \\
\hline $\mathrm{H}$ & -2.7842365736 & -1.2740847145 & -1.0684922929 \\
\hline $\mathrm{H}$ & -3.5915040188 & -2.7781360063 & -0.5325405106 \\
\hline $\mathrm{H}$ & -2.5024160464 & -4.1724521189 & 1.3207996695 \\
\hline $\mathrm{H}$ & -0.8726208074 & -3.7316756378 & 1.8817522836 \\
\hline $\mathrm{H}$ & -1.0968206362 & -4.4070442066 & 0.2463009322 \\
\hline $\mathrm{H}$ & -3.3772271966 & 0.1442008263 & 0.8945764802 \\
\hline $\mathrm{C}$ & -1.6017267029 & 1.7339913211 & -0.4788656202 \\
\hline $\mathrm{H}$ & -1.1957717262 & -0.8805342440 & 2.6508126052 \\
\hline $\mathrm{C}$ & -2.2898850475 & 2.0986519617 & -1.7755755517 \\
\hline $\mathrm{H}$ & -2.8208237463 & 3.0421255551 & -1.6479044185 \\
\hline $\mathrm{H}$ & -1.5417869469 & 2.1989514820 & -2.5640450548 \\
\hline $\mathrm{H}$ & -2.9990855538 & 1.3138140916 & -2.0367250551 \\
\hline $\mathrm{H}$ & 1.6045374208 & 0.8254425474 & 2.1895270032 \\
\hline $\mathrm{O}$ & -2.3833528349 & -1.5033585276 & 1.3468614322 \\
\hline $\mathrm{O}$ & -1.8598086920 & -1.6058905080 & 2.6627313294 \\
\hline $\mathrm{O}$ & -2.4323215709 & 1.7321088402 & 0.6405296100 \\
\hline $\mathrm{O}$ & -3.5781958357 & 0.9378298714 & 0.3651746337 \\
\hline $\mathrm{C}$ & 2.6898175297 & -0.2069273727 & -0.0580158450 \\
\hline $\mathrm{C}$ & 3.2793937586 & -1.5043201043 & -0.6159083173 \\
\hline $\mathrm{H}$ & 2.8385153882 & -2.3503790630 & -0.0855374342 \\
\hline $\mathrm{H}$ & 3.0483060167 & -1.5669643428 & -1.6785672571 \\
\hline $\mathrm{C}$ & 3.3034808054 & 1.0495895206 & -0.6146482128 \\
\hline $\mathrm{H}$ & 3.4273107343 & 1.0310571900 & -1.6974021801 \\
\hline $\mathrm{H}$ & 2.4102880713 & 2.0597395626 & -0.4923827884 \\
\hline $\mathrm{H}$ & 4.1619651796 & 1.4182545442 & -0.0571229727 \\
\hline $\mathrm{Cl}$ & 5.0477788538 & -1.5623583448 & -0.4173761852 \\
\hline $\mathrm{O}$ & 2.7790237781 & -0.3253844746 & 1.3166592427 \\
\hline $\mathrm{O}$ & 2.5463793864 & 0.9317416990 & 1.9361584699 \\
\hline $\mathrm{C}$ & -0.4540842402 & 2.6892962977 & -0.0730427297 \\
\hline $\mathrm{H}$ & -0.0490234205 & 2.4123505896 & 0.9015282166 \\
\hline $\mathrm{Cl}$ & -1.0447864547 & 4.3724691414 & 0.0784123469 \\
\hline $\mathrm{O}$ & 0.5214425686 & 2.5910914707 & -1.0647540816 \\
\hline $\mathrm{O}$ & 1.7358575109 & 3.0204478478 & -0.5419445311 \\
\hline
\end{tabular}




\begin{tabular}{|c|c|c|c|c|}
\hline \multirow[t]{38}{*}{$\mathrm{TS}_{\mathrm{R} 2-7-3}$} & $\mathrm{P}$ & 0.0807000556 & -0.3624045944 & 0.7632914958 \\
\hline & $\mathrm{O}$ & 0.2611370085 & -0.4387939647 & 2.2477695839 \\
\hline & $\mathrm{O}$ & 0.7605163966 & 0.8752269413 & 0.0537729030 \\
\hline & $\mathrm{O}$ & 0.6097760532 & -1.6076645597 & -0.0516382956 \\
\hline & $\mathrm{O}$ & -1.4450062549 & -0.2361944964 & 0.3138924979 \\
\hline & $\mathrm{C}$ & -1.1670038047 & -3.1902898881 & -0.5687361563 \\
\hline & $\mathrm{C}$ & 0.2317987256 & -3.0043303204 & 0.0198908130 \\
\hline & $\mathrm{C}$ & 1.3265079301 & -3.7555529904 & -0.7081584932 \\
\hline & $\mathrm{Cl}$ & -1.2487824265 & -2.6372330413 & -2.2638516378 \\
\hline & $\mathrm{H}$ & -1.9012625190 & -2.6222742920 & -0.0038775219 \\
\hline & $\mathrm{H}$ & -1.4131532610 & -4.2523281976 & -0.5411289545 \\
\hline & $\mathrm{H}$ & 1.0769697296 & -4.8173180147 & -0.7468122985 \\
\hline & $\mathrm{H}$ & 2.2664682656 & -3.6203967586 & -0.1699475748 \\
\hline & $\mathrm{H}$ & 1.4220355272 & -3.3654374374 & -1.7218239254 \\
\hline & $\mathrm{H}$ & -1.2802187459 & 0.4047482297 & 3.0264058821 \\
\hline & $\mathrm{C}$ & -2.2460541870 & 0.9369989810 & 0.5748498354 \\
\hline & $\mathrm{H}$ & -0.3962491510 & -2.2604137631 & 2.5881004476 \\
\hline & $\mathrm{C}$ & -3.6659963219 & 0.4560808978 & 0.7049456752 \\
\hline & $\mathrm{H}$ & -4.3368580781 & 1.1955529215 & 1.1395741036 \\
\hline & $\mathrm{H}$ & -4.0580219147 & 0.5155234307 & -0.6102752426 \\
\hline & $\mathrm{H}$ & -3.7777063088 & -0.5667686912 & 1.0522517600 \\
\hline & $\mathrm{H}$ & 1.8130521556 & 0.5305300324 & 2.6989072424 \\
\hline & $\mathrm{O}$ & 0.2831474212 & -3.4771510980 & 1.3324273512 \\
\hline & $\mathrm{O}$ & -0.8216474159 & -2.9813537751 & 2.0839364344 \\
\hline & $\mathrm{O}$ & -1.7893268185 & 1.6157730243 & 1.7003970850 \\
\hline & $\mathrm{O}$ & -2.1315768277 & 0.8591148123 & 2.8579181661 \\
\hline & $\mathrm{C}$ & 2.2034190173 & 1.1528780351 & 0.0909819149 \\
\hline & $\mathrm{C}$ & 2.7384953457 & 0.5318586725 & -1.1988738412 \\
\hline & $\mathrm{H}$ & 2.6500318784 & -0.5537393088 & -1.1434968770 \\
\hline & $\mathrm{H}$ & 2.1528603448 & 0.9120423083 & -2.0354420095 \\
\hline & $\mathrm{C}$ & 2.3434661488 & 2.6522547786 & 0.1981263303 \\
\hline & $\mathrm{H}$ & 1.9581304550 & 3.1151262802 & -0.7129704082 \\
\hline & $\mathrm{H}$ & 1.7774888127 & 3.0127576346 & 1.0558932066 \\
\hline & $\mathrm{H}$ & 3.3974596967 & 2.9044304092 & 0.3209990181 \\
\hline & $\mathrm{Cl}$ & 4.4480957897 & 0.9410568195 & -1.4874241497 \\
\hline & $\mathrm{O}$ & 2.8022136704 & 0.4312169225 & 1.1185751004 \\
\hline & $\mathrm{O}$ & 2.5713038900 & 1.0585239451 & 2.3724856845 \\
\hline & $\mathrm{C}$ & -2.0639908628 & 1.8962176317 & -0.6709530225 \\
\hline
\end{tabular}




\begin{tabular}{|c|c|c|c|c|}
\hline & $\mathrm{H}$ & -1.4867129587 & 1.3558996281 & -1.4245215844 \\
\hline & $\mathrm{Cl}$ & -1.2640355370 & 3.4275068587 & -0.3209742282 \\
\hline & $\mathrm{O}$ & -3.3286954552 & 2.2392898001 & -1.1855081610 \\
\hline & $\mathrm{O}$ & -3.8953374689 & 1.0452528566 & -1.6285370392 \\
\hline $\mathrm{TS}_{\mathrm{R} 2-7-4}$ & $\mathrm{P}$ & 0.0411553879 & -0.3162819247 & 0.7357678946 \\
\hline & $\mathrm{O}$ & 0.0640841478 & -0.4326976416 & 2.2218815618 \\
\hline & $\mathrm{O}$ & 0.9185965936 & 0.8412620213 & 0.1185196807 \\
\hline & $\mathrm{O}$ & 0.6142342755 & -1.5538279711 & -0.0643935945 \\
\hline & $\mathrm{O}$ & -1.4135468302 & -0.1218915910 & 0.1118763115 \\
\hline & $\mathrm{C}$ & -1.1952913010 & -3.0200379206 & -0.8135739468 \\
\hline & $\mathrm{C}$ & 0.1471778069 & -2.9245318635 & -0.0869344656 \\
\hline & $\mathrm{C}$ & 1.2637876186 & -3.7179320659 & -0.7337462320 \\
\hline & $\mathrm{Cl}$ & -1.0786523385 & -2.4294550638 & -2.4920844204 \\
\hline & $\mathrm{H}$ & -1.9544812710 & -2.4289189188 & -0.3085643210 \\
\hline & $\mathrm{H}$ & -1.4930427630 & -4.0690297023 & -0.8369473934 \\
\hline & $\mathrm{H}$ & 0.9555750620 & -4.7591826564 & -0.8431263338 \\
\hline & $\mathrm{H}$ & 2.1500530549 & -3.6659196911 & -0.0986670561 \\
\hline & $\mathrm{H}$ & 1.4864959468 & -3.2975144199 & -1.7146245414 \\
\hline & $\mathrm{H}$ & -1.1996173185 & 0.6609865606 & 2.9074241922 \\
\hline & $\mathrm{C}$ & -2.2823712564 & 0.9677466176 & 0.4761126306 \\
\hline & $\mathrm{H}$ & -0.6998543349 & -2.2207983078 & 2.4249130685 \\
\hline & $\mathrm{C}$ & -3.6197453028 & 0.3768444318 & 0.8694292151 \\
\hline & $\mathrm{H}$ & -4.3185277972 & 1.1793545324 & 1.1180921218 \\
\hline & $\mathrm{H}$ & -4.0131031075 & -0.2135823031 & 0.0428888322 \\
\hline & $\mathrm{H}$ & -3.4862615796 & -0.2584837783 & 1.7436807716 \\
\hline & $\mathrm{H}$ & 3.0801777855 & -0.0296688715 & -0.4400689864 \\
\hline & $\mathrm{O}$ & 0.0450585092 & -3.4416805943 & 1.2083438287 \\
\hline & $\mathrm{O}$ & -1.1031613446 & -2.9149003682 & 1.8668012199 \\
\hline & $\mathrm{O}$ & -1.7028161491 & 1.7654767711 & 1.4752402860 \\
\hline & $\mathrm{O}$ & -1.9665585197 & 1.2643767142 & 2.7806107493 \\
\hline & $\mathrm{C}$ & 1.7608624684 & 1.8262311392 & 0.7283296498 \\
\hline & $\mathrm{C}$ & 1.7048749011 & 2.9985574122 & -0.2370340793 \\
\hline & $\mathrm{H}$ & 0.4295444392 & 3.3082351269 & -0.2685465078 \\
\hline & $\mathrm{H}$ & 2.2228019219 & 3.8840863664 & 0.1313595169 \\
\hline & $\mathrm{C}$ & 1.3394291949 & 2.2871629435 & 2.1153228379 \\
\hline & $\mathrm{H}$ & 0.3167773807 & 2.6658335479 & 2.0901416738 \\
\hline & $\mathrm{H}$ & 1.4109031100 & 1.4577753216 & 2.8185108933 \\
\hline
\end{tabular}




\begin{tabular}{|c|c|c|c|c|}
\hline & $\mathrm{H}$ & 2.0192257797 & 3.0826636348 & 2.4274259759 \\
\hline & $\mathrm{Cl}$ & 2.0365939482 & 2.7229112280 & -1.9160043596 \\
\hline & $\mathrm{O}$ & 3.0273906539 & 1.2586234212 & 0.9343496289 \\
\hline & $\mathrm{O}$ & 3.5825072212 & 0.7882333574 & -0.2810958370 \\
\hline & $\mathrm{C}$ & -2.3813001150 & 1.9417900766 & -0.7240482781 \\
\hline & $\mathrm{H}$ & -3.0000339015 & 2.7917253857 & -0.4271628417 \\
\hline & $\mathrm{Cl}$ & -3.1378160469 & 1.1410702839 & -2.1158664860 \\
\hline & $\mathrm{O}$ & -1.1495958241 & 2.4110161070 & -1.2315489570 \\
\hline & $\mathrm{O}$ & -0.7514334666 & 3.5326262826 & -0.5169794235 \\
\hline \multirow[t]{28}{*}{$\mathrm{TS}_{\mathrm{R} 2-7-5}$} & $\mathrm{P}$ & 0.0252175489 & -0.3582143843 & 0.2889298274 \\
\hline & $\mathrm{O}$ & 0.3554831703 & -0.0546518409 & 1.6962054949 \\
\hline & $\mathrm{O}$ & -0.9866752369 & -1.5605590928 & 0.0341283768 \\
\hline & $\mathrm{O}$ & -0.6623196707 & 0.8172166747 & -0.5506848989 \\
\hline & $\mathrm{O}$ & 1.2338237363 & -0.7770100059 & -0.6503247667 \\
\hline & $\mathrm{C}$ & -1.4907428104 & 2.9799005080 & 0.0487580109 \\
\hline & $\mathrm{C}$ & -0.3347891928 & 2.2096125707 & -0.6001676969 \\
\hline & $\mathrm{C}$ & 1.0152458624 & 2.5587227736 & -0.0298549479 \\
\hline & $\mathrm{Cl}$ & -1.5386283406 & 2.7464682353 & 1.8137660305 \\
\hline & $\mathrm{H}$ & -2.4342057100 & 2.6289968284 & -0.3669399821 \\
\hline & $\mathrm{H}$ & -1.3611577853 & 4.0430589918 & -0.1556574516 \\
\hline & $\mathrm{H}$ & 1.2913338364 & 3.6003065095 & -0.1900614994 \\
\hline & $\mathrm{H}$ & 1.9195759791 & 1.9047073445 & -0.8580409317 \\
\hline & $\mathrm{H}$ & 1.2009804402 & 2.1750988811 & 0.9709706063 \\
\hline & $\mathrm{H}$ & 1.4607839609 & -2.4904723187 & -2.4328780313 \\
\hline & $\mathrm{C}$ & 2.5497714690 & -1.2556483806 & -0.3873059730 \\
\hline & $\mathrm{H}$ & -1.3072448998 & 1.4615413593 & -3.0253453949 \\
\hline & $\mathrm{C}$ & 2.5612051534 & -2.2616762866 & 0.7470356241 \\
\hline & $\mathrm{H}$ & 3.5830523402 & -2.6106980868 & 0.9138689264 \\
\hline & $\mathrm{H}$ & 2.1770870652 & -1.8072132088 & 1.6609007659 \\
\hline & $\mathrm{H}$ & 1.9379310486 & -3.1093130616 & 0.4595787579 \\
\hline & $\mathrm{H}$ & -1.1965812633 & -0.4491302917 & 2.6910711125 \\
\hline & $\mathrm{O}$ & -0.2376110410 & 2.5616650480 & -1.9584415558 \\
\hline & $\mathrm{O}$ & -1.4825118642 & 2.3140851120 & -2.5974244703 \\
\hline & $\mathrm{O}$ & 2.9885070035 & -1.8016295673 & -1.6057713392 \\
\hline & $\mathrm{O}$ & 2.1780467499 & -2.9157347987 & -1.9356690513 \\
\hline & $\mathrm{C}$ & -2.3775275239 & -1.5128730577 & 0.4880442999 \\
\hline & $\mathrm{C}$ & -3.1654528464 & -1.0752933235 & -0.7476473619 \\
\hline
\end{tabular}




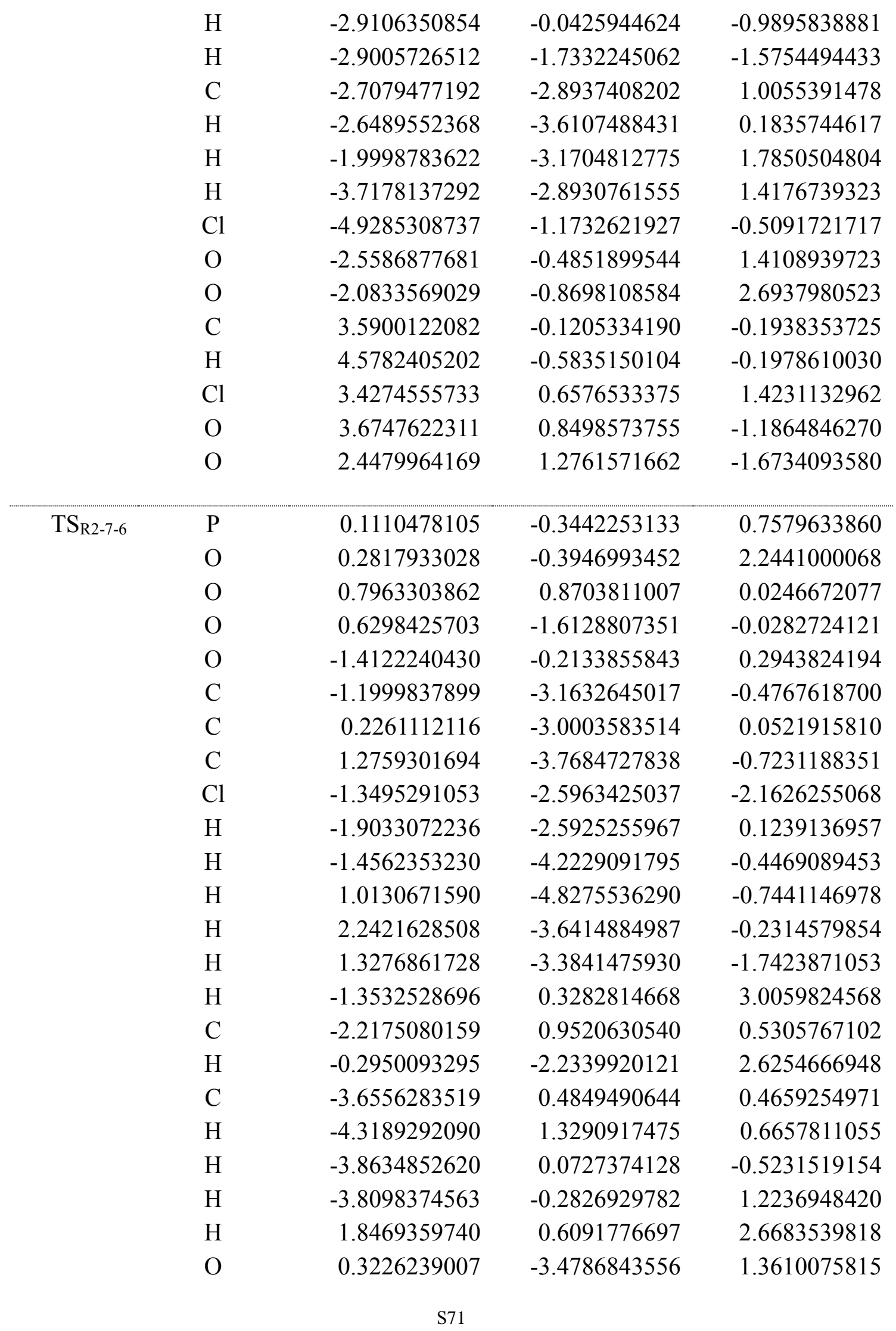




\begin{tabular}{|c|c|c|c|c|}
\hline & $\mathrm{O}$ & -0.7414903424 & -2.9667492806 & 2.1578584449 \\
\hline & $\mathrm{O}$ & -1.8651835619 & 1.5761672957 & 1.7266712480 \\
\hline & $\mathrm{O}$ & -2.2238935112 & 0.7323880778 & 2.8120842820 \\
\hline & $\mathrm{C}$ & 2.2336270599 & 1.1620039263 & 0.0461125810 \\
\hline & $\mathrm{C}$ & 2.7628990159 & 0.5073529756 & -1.2292868882 \\
\hline & $\mathrm{H}$ & 2.6905786641 & -0.5769555115 & -1.1377593502 \\
\hline & $\mathrm{H}$ & 2.1591536374 & 0.8520951246 & -2.0687866543 \\
\hline & $\mathrm{C}$ & 2.3585849793 & 2.6651148166 & 0.1084448398 \\
\hline & $\mathrm{H}$ & 1.9584366161 & 3.0959961727 & -0.8119457567 \\
\hline & $\mathrm{H}$ & 1.7933843203 & 3.0449542931 & 0.9580071466 \\
\hline & $\mathrm{H}$ & 3.4108499705 & 2.9315556758 & 0.2152834464 \\
\hline & $\mathrm{Cl}$ & 4.4636218613 & 0.9276031864 & -1.5548932917 \\
\hline & $\mathrm{O}$ & 2.8402868403 & 0.4726034343 & 1.0919862062 \\
\hline & $\mathrm{O}$ & 2.6013300680 & 1.1321637152 & 2.3278266769 \\
\hline & $\mathrm{C}$ & -1.9039936285 & 2.0321194476 & -0.5345867051 \\
\hline & $\mathrm{H}$ & -2.7397535380 & 3.0274687438 & -0.4202644501 \\
\hline & $\mathrm{Cl}$ & -1.9198980444 & 1.4351934424 & -2.1795207295 \\
\hline & $\mathrm{O}$ & -0.9099868698 & 2.9231308577 & -0.2597883852 \\
\hline & $\mathrm{O}$ & -1.9097720054 & 3.9982246220 & -0.2043787938 \\
\hline \multirow[t]{18}{*}{$\mathrm{TS}_{\mathrm{R} 2-9-1}$} & $\mathrm{P}$ & 0.7594861539 & -0.0414942311 & -0.2350983944 \\
\hline & $\mathrm{O}$ & 0.9693961356 & -0.0489921436 & -1.7009336856 \\
\hline & $\mathrm{O}$ & 2.0461242595 & 0.1990553718 & 0.6645006965 \\
\hline & $\mathrm{O}$ & -0.2601138239 & 1.0206472146 & 0.3404857142 \\
\hline & $\mathrm{O}$ & 0.1785941603 & -1.3837022377 & 0.4078797497 \\
\hline & $\mathrm{C}$ & -0.7157363553 & 2.2514199812 & -0.2120463927 \\
\hline & $\mathrm{C}$ & 0.3636595380 & 3.3072995257 & -0.1539409971 \\
\hline & $\mathrm{H}$ & -2.6131632799 & 0.8729889187 & -1.9536504556 \\
\hline & $\mathrm{H}$ & 0.6655460801 & 3.4492596783 & 0.8834632288 \\
\hline & $\mathrm{H}$ & -0.0125715161 & 4.2443707318 & -0.5706270538 \\
\hline & $\mathrm{H}$ & 1.2261539210 & 2.9859825221 & -0.7404564286 \\
\hline & $\mathrm{H}$ & -2.6199110313 & -2.5490218576 & 2.1422911571 \\
\hline & $\mathrm{C}$ & -0.9033810206 & -2.2111148719 & -0.0449390149 \\
\hline & $\mathrm{H}$ & 0.1572145333 & 1.3240371418 & -2.5294027031 \\
\hline & $\mathrm{C}$ & -0.4233641060 & -3.1120829823 & -1.1671946978 \\
\hline & $\mathrm{H}$ & -0.0492913534 & -2.4916456459 & -1.9835766160 \\
\hline & $\mathrm{H}$ & 0.3843204916 & -3.7372922733 & -0.7803456167 \\
\hline & $\mathrm{H}$ & -1.2447740249 & -3.7375078291 & -1.5209389168 \\
\hline
\end{tabular}




$\begin{array}{lrrr}\mathrm{H} & 1.6040937615 & -2.3689835724 & 1.4356132528 \\ \mathrm{O} & -1.2444991079 & 2.0588002128 & -1.5042848344 \\ \mathrm{O} & -0.2796233436 & 2.2076919354 & -2.5318520402 \\ \mathrm{O} & -1.1925477051 & -3.0383161224 & 1.0309235304 \\ \mathrm{O} & -1.7021213700 & -2.2324919920 & 2.0910917475 \\ \mathrm{C} & 3.2792279937 & -0.5224964121 & 0.4547183942 \\ \mathrm{C} & 3.9193992691 & -0.1503744099 & -0.8877869487 \\ \mathrm{H} & 4.9122504537 & -0.5963867171 & -0.9386493779 \\ \mathrm{H} & 3.2982784760 & -0.5000776129 & -1.7115765863 \\ \mathrm{Cl} & 4.0955797097 & 1.6163313636 & -1.0582249784 \\ \mathrm{O} & 2.9982764746 & -1.8870093213 & 0.2754244360 \\ \mathrm{O} & 2.5730723066 & -2.4639793384 & 1.5019272207 \\ \mathrm{C} & -2.1362290798 & -1.3554374139 & -0.4431217906 \\ \mathrm{H} & -2.3861771923 & -0.3840467926 & 0.4295940257 \\ \mathrm{Cl} & -3.6315126426 & -2.3086684528 & -0.4037618259 \\ \mathrm{O} & -1.8712009870 & -0.8040812742 & -1.6583008324 \\ \mathrm{O} & -2.9754330721 & -0.0258221934 & -2.0934704436 \\ \mathrm{C} & 4.1460134024 & -0.2244318108 & 1.6561218106 \\ \mathrm{H} & 5.0596367855 & -0.8194249740 & 1.5896271543 \\ \mathrm{H} & 4.3981897423 & 0.8371983247 & 1.6643911405 \\ \mathrm{H} & 3.6135738050 & -0.4820957497 & 2.5702614249 \\ \mathrm{C} & -1.9914620806 & 2.5856877192 & 0.5953699663 \\ \mathrm{H} & -2.4342293703 & 3.4880571307 & 0.1697262695 \\ \mathrm{Cl} & -1.6326586152 & 2.9032800856 & 2.3059582844 \\ \mathrm{O} & -3.0036763620 & 1.6213531469 & 0.4362617742 \\ \mathrm{O} & -2.7866130132 & 0.4777082277 & 1.1805616531\end{array}$

$\mathrm{TS}_{\mathrm{R} 2-9-2}$

\section{P}

O

$\mathrm{O}$

$\mathrm{O}$

O

C

C

$\mathrm{H}$

$\mathrm{H}$

$\mathrm{H}$

$\mathrm{H}$

P

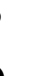

O

O

C

C

H

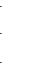

H

0.0871490093
-0.2404419549
0.6644390575
-1.1294938251
1.1557570119
-2.2243561091
-1.9872447558
2.3958175543
-2.7904906423
-1.0313974007
-1.9770611575

$\begin{array}{rr}-0.3836918344 & -0.3781148375 \\ -0.3131614837 & -1.8179852849 \\ 0.9608615644 & 0.2726178349 \\ -0.6962924419 & 0.6008013650 \\ -1.4745359512 & 0.0453057224 \\ -1.6133890240 & 0.3823396840 \\ -2.6153423363 & -0.7277345367 \\ 1.2264727490 & 1.9880697064 \\ -3.3524822851 & -0.7062300751 \\ -3.1195240118 & -0.5633771674 \\ -2.1046792312 & -1.6916034929\end{array}$




\begin{tabular}{|c|c|c|c|}
\hline $\mathrm{H}$ & 0.9655883194 & -1.5578349985 & -2.7142471345 \\
\hline $\mathrm{C}$ & 2.5020139280 & -1.5641532311 & -0.4747621490 \\
\hline $\mathrm{H}$ & -1.7414646382 & -1.3515880547 & 3.0005391491 \\
\hline $\mathrm{C}$ & 2.9462636967 & -2.9846521237 & -0.2210159155 \\
\hline $\mathrm{H}$ & 3.9281810145 & -3.1410789045 & -0.6668530008 \\
\hline $\mathrm{H}$ & 2.9992869798 & -3.1587331599 & 0.8559067273 \\
\hline $\mathrm{H}$ & 2.2245431623 & -3.6650358183 & -0.6724083202 \\
\hline $\mathrm{H}$ & 2.6656753652 & 3.4013702738 & 1.0441781700 \\
\hline $\mathrm{O}$ & -2.3573514642 & -2.3623637777 & 1.5476948540 \\
\hline $\mathrm{O}$ & -2.6307961437 & -1.4897881603 & 2.6368593430 \\
\hline $\mathrm{O}$ & 2.5383446420 & -1.1637684532 & -1.8106860897 \\
\hline $\mathrm{O}$ & 1.7901618809 & -2.0750616443 & -2.6012725535 \\
\hline $\mathrm{C}$ & -0.0012400501 & 2.2451646813 & 0.3119646612 \\
\hline $\mathrm{C}$ & -0.8345143141 & 2.4594923053 & -0.9493057184 \\
\hline $\mathrm{H}$ & -0.3591525476 & 2.1391958975 & -1.8758731154 \\
\hline $\mathrm{H}$ & -1.9678248037 & 1.6882005461 & -0.8894746977 \\
\hline $\mathrm{Cl}$ & -1.5350738466 & 4.0411195039 & -1.0736127284 \\
\hline $\mathrm{O}$ & 1.0157089129 & 3.2008076648 & 0.2035500163 \\
\hline $\mathrm{O}$ & 1.7977597582 & 3.1602068218 & 1.4045508909 \\
\hline $\mathrm{C}$ & 3.3342215130 & -0.4587582931 & 0.2214886798 \\
\hline $\mathrm{H}$ & 3.0771131581 & 0.5091181285 & -0.2141826015 \\
\hline $\mathrm{Cl}$ & 5.0918814595 & -0.6832879715 & -0.0651801161 \\
\hline $\mathrm{O}$ & 3.0215128820 & -0.4922323250 & 1.5682518611 \\
\hline $\mathrm{O}$ & 3.2631758657 & 0.7960242827 & 2.1110070761 \\
\hline $\mathrm{C}$ & -0.7868550475 & 2.3531340723 & 1.6049532981 \\
\hline $\mathrm{H}$ & -0.1303777003 & 2.1602193472 & 2.4537049629 \\
\hline $\mathrm{H}$ & -1.2056531196 & 3.3570721277 & 1.6921729982 \\
\hline $\mathrm{H}$ & -1.5951301211 & 1.6191366248 & 1.5989196791 \\
\hline $\mathrm{C}$ & -3.4163986064 & -0.6673055768 & 0.1500518586 \\
\hline $\mathrm{H}$ & -3.5115289495 & 0.0387434044 & 0.9726300892 \\
\hline $\mathrm{Cl}$ & -4.9401851633 & -1.5738947231 & 0.0171693913 \\
\hline $\mathrm{O}$ & -3.1865249583 & 0.0297655786 & -1.0526175032 \\
\hline $\mathrm{O}$ & -3.0953858515 & 1.3824802411 & -0.8099089803 \\
\hline $\mathrm{P}$ & 0.1920268353 & -0.0264899339 & 0.8439558960 \\
\hline $\mathrm{O}$ & 0.1203455464 & 0.7762178011 & 2.0874022645 \\
\hline $\mathrm{O}$ & 1.4141160788 & -1.0348585883 & 0.8488620960 \\
\hline $\mathrm{O}$ & -1.0107208144 & -1.0409182532 & 0.5986418331 \\
\hline
\end{tabular}




\begin{tabular}{|c|c|c|c|}
\hline $\mathrm{O}$ & 0.3300311621 & 0.8180034425 & -0.5030864625 \\
\hline $\mathrm{C}$ & -2.3443590694 & -0.8522721013 & 0.1136636264 \\
\hline $\mathrm{C}$ & -3.1619831246 & -2.0782701343 & 0.4625985104 \\
\hline $\mathrm{H}$ & 1.9927587092 & 1.2764582797 & 2.5823564225 \\
\hline $\mathrm{H}$ & -2.6780301766 & -2.9708714698 & 0.0716149225 \\
\hline $\mathrm{H}$ & -4.1581846950 & -1.9775142789 & 0.0279913311 \\
\hline $\mathrm{H}$ & -3.2410135207 & -2.1397959068 & 1.5492212771 \\
\hline $\mathrm{H}$ & -2.0309813219 & 2.3892571085 & -1.2118935329 \\
\hline $\mathrm{C}$ & 0.7303505636 & 2.1951130104 & -0.6971025274 \\
\hline $\mathrm{H}$ & -1.8809214931 & 1.6074931826 & 1.3832138578 \\
\hline $\mathrm{C}$ & 1.4082505544 & 2.2145135600 & -2.0502947417 \\
\hline $\mathrm{H}$ & 1.5986406729 & 3.2430950486 & -2.3547461704 \\
\hline $\mathrm{H}$ & 2.3533390897 & 1.6674665938 & -1.9857066492 \\
\hline $\mathrm{H}$ & 0.7540693785 & 1.7367658062 & -2.7803689191 \\
\hline $\mathrm{H}$ & 3.5270639509 & -0.0186534279 & 0.0652475378 \\
\hline $\mathrm{O}$ & -2.9801742009 & 0.1864789916 & 0.8026775559 \\
\hline $\mathrm{O}$ & -2.3121787123 & 1.4100564206 & 0.5302238248 \\
\hline $\mathrm{O}$ & -0.3923959185 & 3.0140662334 & -0.5963631020 \\
\hline $\mathrm{O}$ & -1.2616192354 & 2.7506940305 & -1.6903328437 \\
\hline $\mathrm{C}$ & 1.8388152108 & -1.9708945191 & -0.1708616461 \\
\hline $\mathrm{C}$ & 1.5128571378 & -3.3394439549 & 0.4258531011 \\
\hline $\mathrm{H}$ & 2.0610633107 & -3.4495433190 & 1.3617425232 \\
\hline $\mathrm{H}$ & 0.4373798563 & -3.3827084761 & 0.6067370666 \\
\hline $\mathrm{Cl}$ & 1.9520490892 & -4.6888152472 & -0.6497783958 \\
\hline $\mathrm{O}$ & 3.2335123544 & -1.8684269024 & -0.1832730443 \\
\hline $\mathrm{O}$ & 3.5813404371 & -0.5981197090 & -0.7174453455 \\
\hline $\mathrm{C}$ & 1.5951628103 & 2.6923172672 & 0.4928924944 \\
\hline $\mathrm{H}$ & 0.9479915757 & 2.9177159363 & 1.3405311099 \\
\hline $\mathrm{Cl}$ & 2.4648482795 & 4.2001990562 & 0.0931031684 \\
\hline $\mathrm{O}$ & 2.4722120099 & 1.6644356560 & 0.8215646502 \\
\hline $\mathrm{O}$ & 2.7754832056 & 1.7339254212 & 2.2061695302 \\
\hline $\mathrm{C}$ & 1.2774758073 & -1.7483826984 & -1.5590134267 \\
\hline $\mathrm{H}$ & 1.6883244229 & -2.4702061306 & -2.2635407900 \\
\hline $\mathrm{H}$ & -0.0508429485 & -2.1509325339 & -1.5928613765 \\
\hline $\mathrm{H}$ & 1.2609010820 & -0.7244755379 & -1.9189922391 \\
\hline $\mathrm{C}$ & -2.2238505662 & -0.5768401314 & -1.4090433619 \\
\hline $\mathrm{H}$ & -1.4332523347 & 0.1507810470 & -1.6007920391 \\
\hline $\mathrm{Cl}$ & -3.7253519220 & 0.0947641322 & -2.0624346025 \\
\hline
\end{tabular}




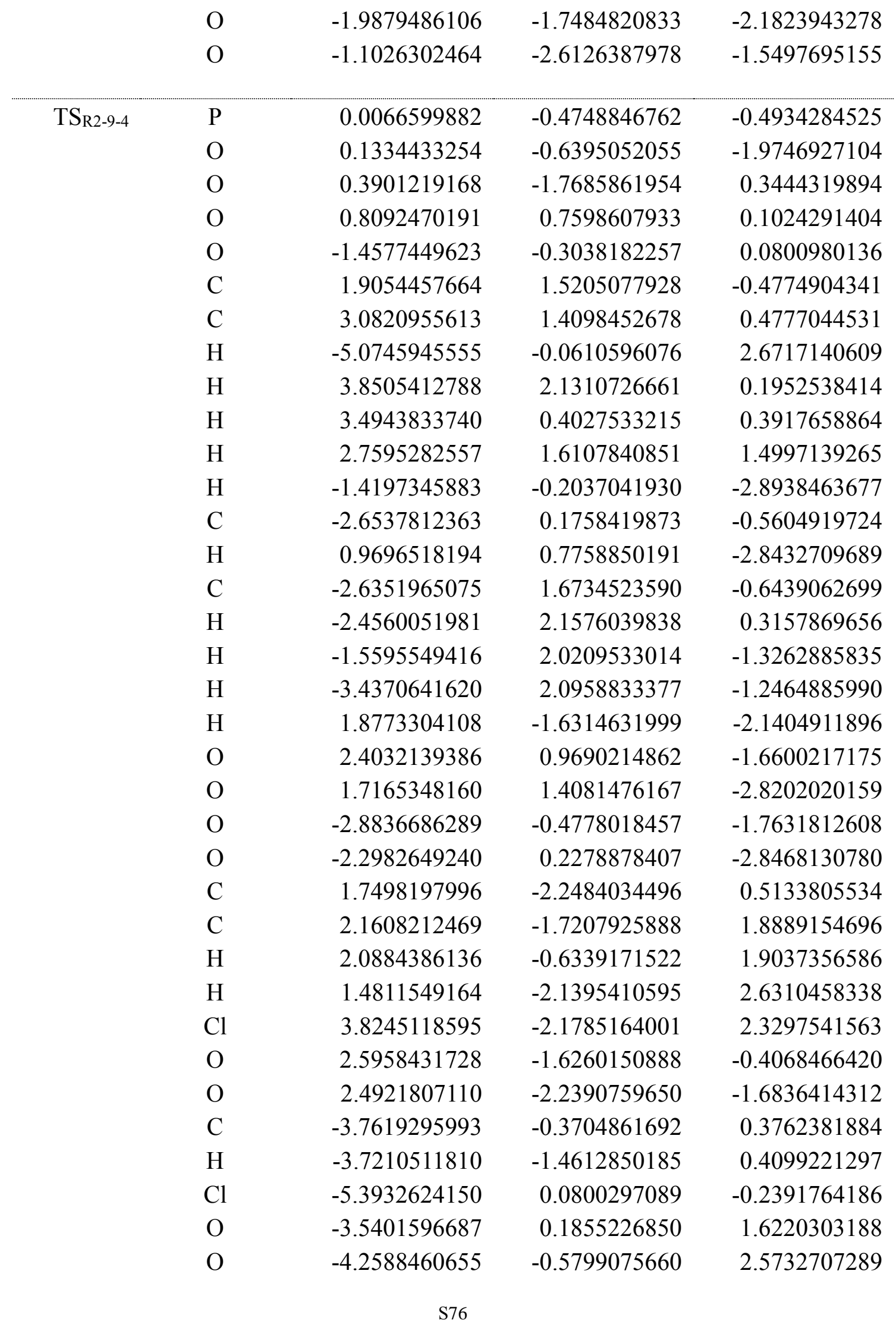




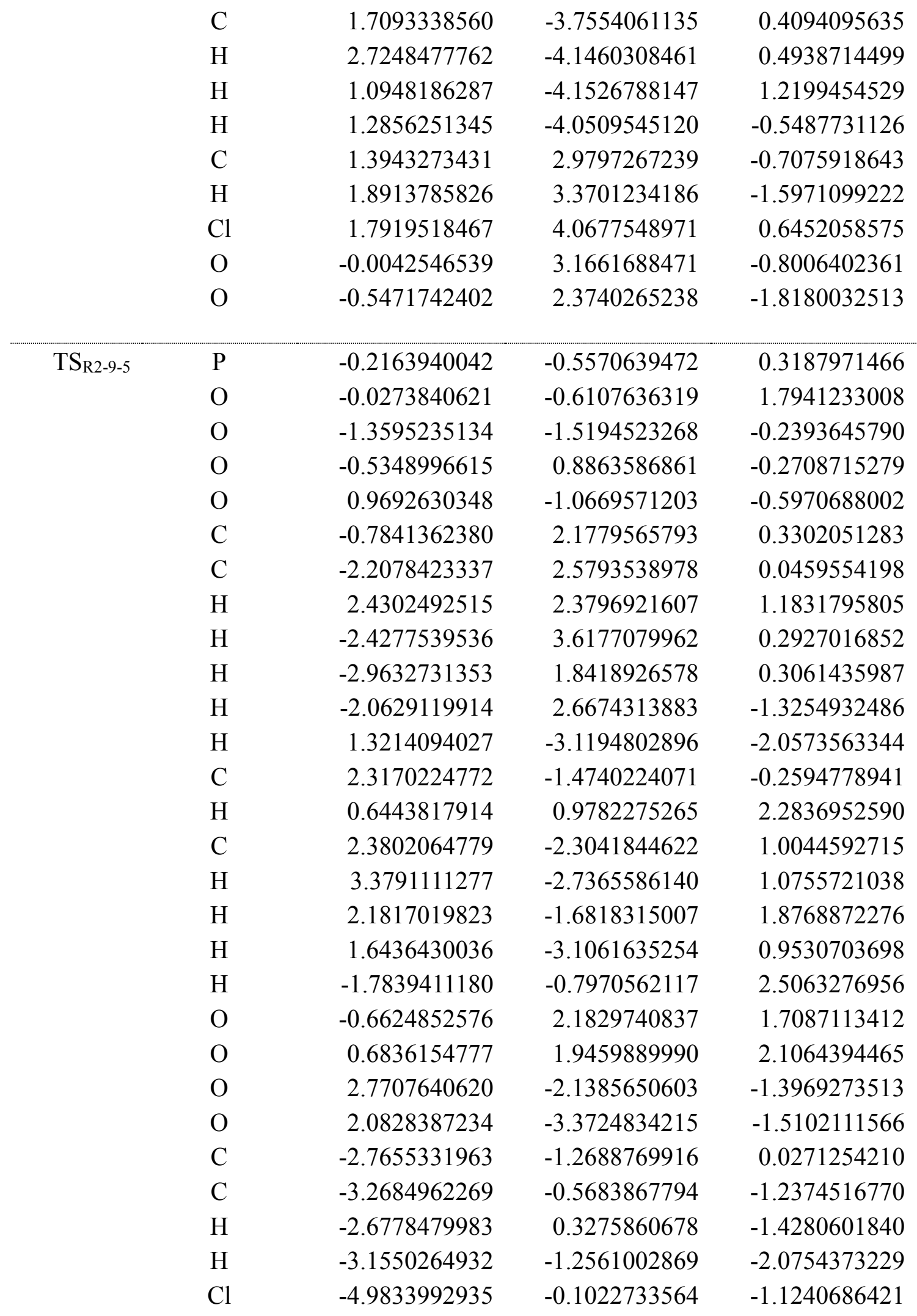




\begin{tabular}{|c|c|c|c|c|}
\hline & $\mathrm{O}$ & -2.8949887423 & -0.3392202209 & 1.0655000617 \\
\hline & $\mathrm{O}$ & -2.7345232388 & -0.9640259332 & 2.3314518664 \\
\hline & $\mathrm{C}$ & 3.1565271082 & -0.1804771086 & -0.2328287636 \\
\hline & $\mathrm{H}$ & 3.0825407825 & 0.3363552009 & -1.1927993922 \\
\hline & $\mathrm{Cl}$ & 4.8914344637 & -0.5329085610 & 0.0431397345 \\
\hline & $\mathrm{O}$ & 2.6171938457 & 0.5794339328 & 0.7845784818 \\
\hline & $\mathrm{O}$ & 3.0414999742 & 1.9268897876 & 0.5699080355 \\
\hline & $\mathrm{C}$ & -3.4191505725 & -2.6028863733 & 0.3013606066 \\
\hline & $\mathrm{H}$ & -4.4708106514 & -2.4428269010 & 0.5454231848 \\
\hline & $\mathrm{H}$ & -3.3417474367 & -3.2288693875 & -0.5900837498 \\
\hline & $\mathrm{H}$ & -2.9254077293 & -3.0950718828 & 1.1374640752 \\
\hline & $\mathrm{C}$ & 0.2142508368 & 3.1230996915 & -0.4354067422 \\
\hline & $\mathrm{H}$ & 1.0681382606 & 2.5441179533 & -0.7945762488 \\
\hline & $\mathrm{Cl}$ & 0.8067082742 & 4.4624814813 & 0.5537190877 \\
\hline & $\mathrm{O}$ & -0.4612121570 & 3.7744457359 & -1.5011006585 \\
\hline & $\mathrm{O}$ & -1.2670924031 & 2.8464520446 & -2.1539318569 \\
\hline \multirow[t]{21}{*}{$\mathrm{TS}_{\mathrm{R} 2-9-6}$} & $\mathrm{P}$ & -0.1943494192 & -0.3657502876 & 0.2790664592 \\
\hline & $\mathrm{O}$ & -0.1257802620 & -0.2966187304 & 1.7610426185 \\
\hline & $\mathrm{O}$ & -1.1672094881 & -1.4854999782 & -0.3002033670 \\
\hline & $\mathrm{O}$ & -0.6702961212 & 0.9661495149 & -0.4596754152 \\
\hline & $\mathrm{O}$ & 1.1519043360 & -0.6950705620 & -0.4843524584 \\
\hline & $\mathrm{C}$ & -0.8215306686 & 2.3001997144 & 0.0363875290 \\
\hline & $\mathrm{C}$ & -2.2740705665 & 2.7125835832 & -0.1247667276 \\
\hline & $\mathrm{H}$ & 2.8263466139 & 2.2333679092 & 1.1464785158 \\
\hline & $\mathrm{H}$ & -2.3855523252 & 3.7677596307 & 0.1283775384 \\
\hline & $\mathrm{H}$ & -2.8810196585 & 2.0947268882 & 0.5409918301 \\
\hline & $\mathrm{H}$ & -2.5886345801 & 2.5530090522 & -1.1582183335 \\
\hline & $\mathrm{H}$ & 1.2582530423 & -2.6279689051 & -2.1178402847 \\
\hline & $\mathrm{C}$ & 2.2960367668 & -1.4518592206 & -0.0197318392 \\
\hline & $\mathrm{H}$ & 0.7522839803 & 1.3195497100 & 2.0735096331 \\
\hline & $\mathrm{C}$ & 1.9845883588 & -2.3862699257 & 1.1308312396 \\
\hline & $\mathrm{H}$ & 2.8442261464 & -3.0431917345 & 1.2690227646 \\
\hline & $\mathrm{H}$ & 1.8039436195 & -1.8164486550 & 2.0424457560 \\
\hline & $\mathrm{H}$ & 1.1090505059 & -2.9912687377 & 0.8901211986 \\
\hline & $\mathrm{H}$ & -1.8068631907 & -0.7708627986 & 2.4398814523 \\
\hline & $\mathrm{O}$ & -0.5688998813 & 2.4511608491 & 1.3927767514 \\
\hline & $\mathrm{O}$ & 0.8001862791 & 2.2233854526 & 1.6902786904 \\
\hline
\end{tabular}




\begin{tabular}{|c|c|c|c|c|}
\hline & $\mathrm{O}$ & 2.7753820989 & -2.1013959739 & -1.1573941326 \\
\hline & $\mathrm{O}$ & 1.8501429220 & -3.1125525520 & -1.5196723250 \\
\hline & $\mathrm{C}$ & -2.6100114450 & -1.4456144843 & -0.0585254896 \\
\hline & $\mathrm{C}$ & -3.1912036643 & -0.8451049297 & -1.3382236556 \\
\hline & $\mathrm{H}$ & -2.8355616212 & 0.1788437387 & -1.4513116317 \\
\hline & $\mathrm{H}$ & -2.8621390772 & -1.4471430280 & -2.1851049462 \\
\hline & $\mathrm{Cl}$ & -4.9718741965 & -0.8213471554 & -1.3281853706 \\
\hline & $\mathrm{O}$ & -2.9069791182 & -0.5235376331 & 0.9472268071 \\
\hline & $\mathrm{O}$ & -2.7112444092 & -1.0910775674 & 2.2351369229 \\
\hline & $\mathrm{C}$ & 3.3669389418 & -0.3849516301 & 0.2844066173 \\
\hline & $\mathrm{H}$ & 3.5494111532 & 0.2242778335 & -0.6031956763 \\
\hline & $\mathrm{Cl}$ & 4.9282164750 & -1.1423706969 & 0.7301401021 \\
\hline & $\mathrm{O}$ & 2.8556345372 & 0.3779451335 & 1.3200575954 \\
\hline & $\mathrm{O}$ & 3.5554768082 & 1.6159095270 & 1.3436871222 \\
\hline & $\mathrm{C}$ & -3.0449999653 & -2.8621903315 & 0.2338635293 \\
\hline & $\mathrm{H}$ & -4.1068835986 & -2.8685511175 & 0.4830402684 \\
\hline & $\mathrm{H}$ & -2.8730838825 & -3.4775687368 & -0.6519550265 \\
\hline & $\mathrm{H}$ & -2.4746103761 & -3.2593466496 & 1.0718626301 \\
\hline & $\mathrm{C}$ & 0.1070497854 & 3.1876700680 & -0.8191461543 \\
\hline & $\mathrm{H}$ & -0.4954138927 & 3.5351387520 & -1.9230493073 \\
\hline & $\mathrm{Cl}$ & 1.6932214655 & 2.5830763479 & -1.2482350687 \\
\hline & $\mathrm{O}$ & 0.0678826106 & 4.5005037942 & -0.4586696245 \\
\hline & $\mathrm{O}$ & -0.5808956686 & 4.8189260621 & -1.7370154373 \\
\hline $\mathrm{TS}_{\mathrm{R} 2-10-1}$ & $\mathrm{P}$ & -0.2045289377 & 0.1937344770 & -0.5685312888 \\
\hline & $\mathrm{O}$ & -0.4757896263 & 1.1479865096 & -1.6596234097 \\
\hline & $\mathrm{O}$ & 0.4595041147 & -1.1544576913 & -1.0639110459 \\
\hline & $\mathrm{O}$ & 0.8001566087 & 0.6736205763 & 0.5840345493 \\
\hline & $\mathrm{O}$ & -1.5056562172 & -0.1936667802 & 0.2865816411 \\
\hline & $\mathrm{C}$ & 1.7538484259 & 2.9341053737 & -0.4470570599 \\
\hline & $\mathrm{C}$ & 0.9116213637 & 1.9973599121 & 1.1135208616 \\
\hline & $\mathrm{C}$ & -0.4043742977 & 2.7566515376 & 1.1796619248 \\
\hline & $\mathrm{Cl}$ & 3.1376097735 & 2.0511588997 & -0.9563508580 \\
\hline & $\mathrm{H}$ & 1.9943708149 & 3.8906330894 & 0.0053372327 \\
\hline & $\mathrm{H}$ & 0.9284610053 & 2.8642020918 & -1.1531065482 \\
\hline & $\mathrm{H}$ & -0.9442900681 & 2.7409678868 & 0.2317116337 \\
\hline & $\mathrm{H}$ & -0.1959111921 & 3.7825061952 & 1.4842894817 \\
\hline & $\mathrm{H}$ & -1.0129989856 & 2.2742582701 & 1.9505343625 \\
\hline
\end{tabular}




\begin{tabular}{|c|c|c|c|c|}
\hline & $\mathrm{C}$ & 1.1574774250 & -2.1432514691 & -0.2641663469 \\
\hline & $\mathrm{H}$ & 1.1588014360 & -3.0264165708 & -0.9083434760 \\
\hline & $\mathrm{C}$ & 0.4534830524 & -2.4549017077 & 1.0437523503 \\
\hline & $\mathrm{H}$ & 0.4532509876 & -1.5875502188 & 1.7094901392 \\
\hline & $\mathrm{H}$ & -0.5790979990 & -2.7601878578 & 0.8592215037 \\
\hline & $\mathrm{H}$ & 0.9797194479 & -3.2737503140 & 1.5394016030 \\
\hline & $\mathrm{C}$ & 2.5972851241 & -1.6632653206 & -0.0964429019 \\
\hline & $\mathrm{H}$ & 2.9891437537 & -1.3230924489 & -1.0550965435 \\
\hline & $\mathrm{H}$ & 2.6748238179 & -0.8645903236 & 0.6418183890 \\
\hline & $\mathrm{Cl}$ & 3.6253899351 & -3.0256406679 & 0.4549776686 \\
\hline & $\mathrm{C}$ & -2.7995971386 & -0.2079189034 & -0.3623483271 \\
\hline & $\mathrm{H}$ & -2.9445637340 & 0.7583784924 & -0.8582319155 \\
\hline & $\mathrm{C}$ & -2.8859742358 & -1.3419160202 & -1.3665212895 \\
\hline & $\mathrm{H}$ & -3.8866218322 & -1.3786633863 & -1.8028380528 \\
\hline & $\mathrm{H}$ & -2.1636169714 & -1.1949521476 & -2.1736237837 \\
\hline & $\mathrm{H}$ & -2.6790747064 & -2.2973623477 & -0.8733065766 \\
\hline & $\mathrm{C}$ & -3.7702827501 & -0.3426035672 & 0.8025768303 \\
\hline & $\mathrm{H}$ & -3.6657887824 & -1.3195690129 & 1.2786536858 \\
\hline & $\mathrm{H}$ & -3.5918010085 & 0.4430651504 & 1.5368288570 \\
\hline & $\mathrm{Cl}$ & -5.4609032507 & -0.1827154461 & 0.2405561321 \\
\hline & $\mathrm{O}$ & 1.7960186473 & 2.1398957400 & 1.9530075776 \\
\hline $\mathrm{TS}_{\mathrm{R} 2-10-2}$ & $\mathrm{P}$ & -0.0312264737 & -0.1530004085 & 1.4097725163 \\
\hline & $\mathrm{O}$ & -0.3148823760 & 0.7032153582 & 2.5655223416 \\
\hline & $\mathrm{O}$ & -1.2116175188 & -1.1834809738 & 0.9863683063 \\
\hline & $\mathrm{O}$ & 0.2935227474 & 0.5897116178 & 0.0155587708 \\
\hline & $\mathrm{O}$ & 1.2016027168 & -1.1219059668 & 1.5838011968 \\
\hline & $\mathrm{C}$ & 0.1241813421 & 3.0038684054 & 0.1319497742 \\
\hline & $\mathrm{C}$ & -0.4825270576 & 1.7141928724 & -0.4525329626 \\
\hline & $\mathrm{C}$ & -0.4688343174 & 1.6260005165 & -1.9673996931 \\
\hline & $\mathrm{Cl}$ & 1.8139290592 & 3.2181173257 & -0.4045702738 \\
\hline & $\mathrm{H}$ & 0.1157827693 & 2.9243327946 & 1.2197214968 \\
\hline & $\mathrm{H}$ & -0.4501530732 & 3.8680536892 & -0.2002373276 \\
\hline & $\mathrm{H}$ & 0.5537068471 & 1.5881141497 & -2.3460501988 \\
\hline & $\mathrm{H}$ & -0.9840554743 & 2.4947873738 & -2.3831191016 \\
\hline & $\mathrm{H}$ & -1.0039115547 & 0.7217397555 & -2.2720258912 \\
\hline & $\mathrm{C}$ & -2.4196043849 & -0.7065333852 & 0.4257774846 \\
\hline & $\mathrm{H}$ & -2.2264921755 & 0.4037900256 & 0.0001489387 \\
\hline
\end{tabular}




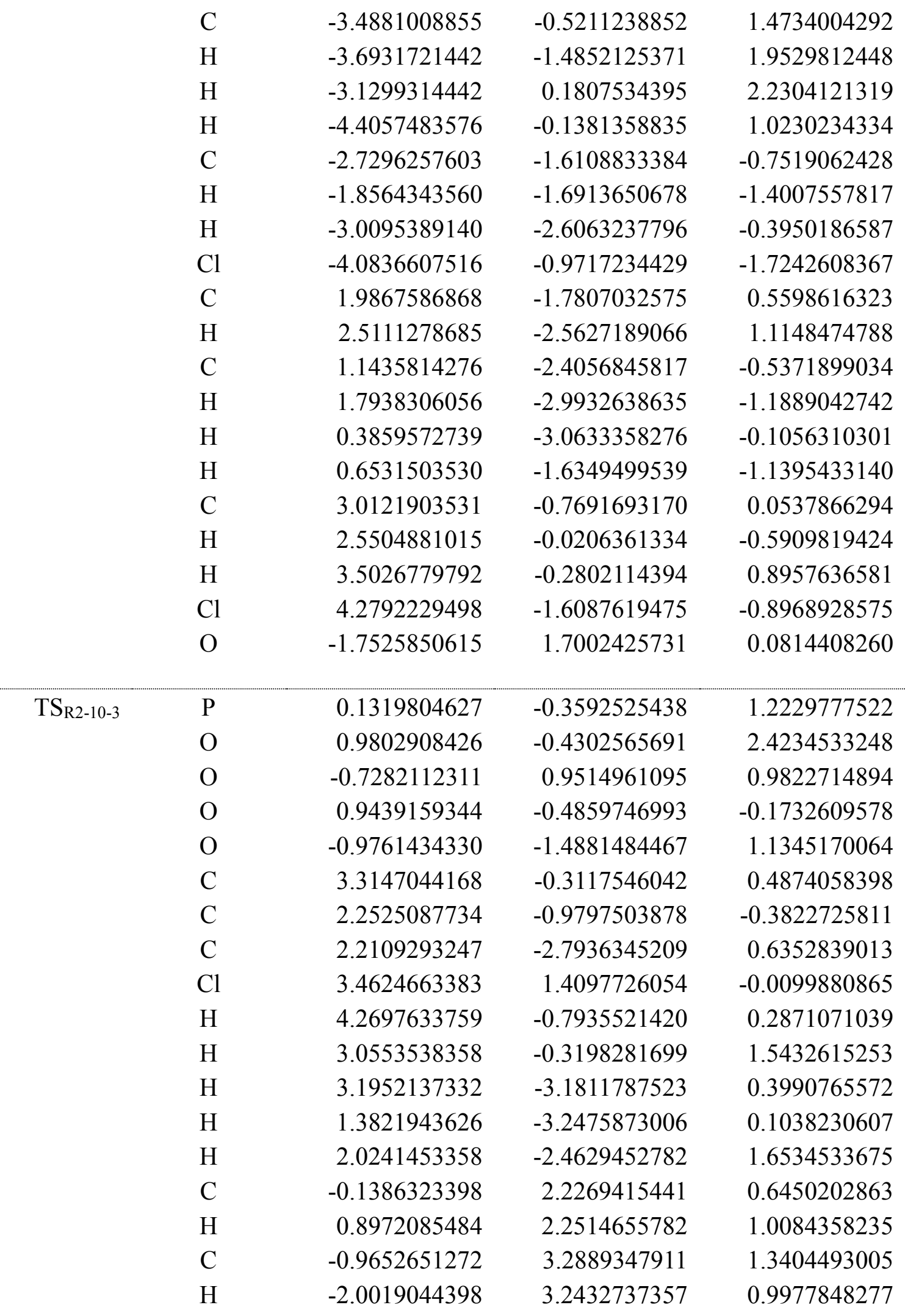




\begin{tabular}{|c|c|c|c|c|}
\hline & $\mathrm{H}$ & -0.9384658570 & 3.1317178281 & 2.4203619902 \\
\hline & $\mathrm{H}$ & -0.5626959281 & 4.2808385555 & 1.1177101176 \\
\hline & $\mathrm{C}$ & -0.0872959984 & 2.3594176192 & -0.8699531178 \\
\hline & $\mathrm{H}$ & 0.2750363116 & 3.3527127431 & -1.1415565726 \\
\hline & $\mathrm{H}$ & 0.5616877880 & 1.5997282778 & -1.3046087112 \\
\hline & $\mathrm{Cl}$ & -1.7101113036 & 2.1498075351 & -1.6066865446 \\
\hline & $\mathrm{C}$ & -1.8996428285 & -1.5220966633 & 0.0075708122 \\
\hline & $\mathrm{H}$ & -1.5095515799 & -0.8930409342 & -0.8010891362 \\
\hline & $\mathrm{C}$ & -2.0034168302 & -2.9651611881 & -0.4358936688 \\
\hline & $\mathrm{H}$ & -2.7185146744 & -3.0554540158 & -1.2560135171 \\
\hline & $\mathrm{H}$ & -1.0298766754 & -3.3188775933 & -0.7829951929 \\
\hline & $\mathrm{H}$ & -2.3339309681 & -3.5917782390 & 0.3977832725 \\
\hline & $\mathrm{C}$ & -3.2005671677 & -0.9234156523 & 0.5268416596 \\
\hline & $\mathrm{H}$ & -3.6062751725 & -1.5354339923 & 1.3346975429 \\
\hline & $\mathrm{H}$ & -3.0296678763 & 0.0966393468 & 0.8707495297 \\
\hline & $\mathrm{Cl}$ & -4.4274621807 & -0.8498078704 & -0.7750107029 \\
\hline & $\mathrm{O}$ & 2.4973713876 & -1.4007565462 & -1.5020073018 \\
\hline $\mathrm{TS}_{\mathrm{R} 2-10-4}$ & $\mathrm{P}$ & 0.1335871418 & -0.6389251701 & 1.1178172988 \\
\hline & $\mathrm{O}$ & 0.9748442399 & -0.9495955315 & 2.2805630087 \\
\hline & $\mathrm{O}$ & -0.4826277884 & 0.8298665163 & 1.0174371303 \\
\hline & $\mathrm{O}$ & 0.9154639356 & -0.6865001659 & -0.3002971007 \\
\hline & $\mathrm{O}$ & -1.1504571509 & -1.5556922787 & 0.9983114686 \\
\hline & $\mathrm{C}$ & 2.0596594733 & -1.3952550479 & -2.2345999703 \\
\hline & $\mathrm{C}$ & 1.3716311052 & -1.8770890945 & -0.9561012569 \\
\hline & $\mathrm{C}$ & 2.3738605584 & -2.6485312423 & -0.0895823713 \\
\hline & $\mathrm{Cl}$ & 0.9827230718 & -0.4235881787 & -3.2797363256 \\
\hline & $\mathrm{H}$ & 2.3713250913 & -2.2672013887 & -2.8098524483 \\
\hline & $\mathrm{H}$ & 2.9219249961 & -0.7750582917 & -1.9874526081 \\
\hline & $\mathrm{H}$ & 3.1985553731 & -1.9837440662 & 0.1789258486 \\
\hline & $\mathrm{H}$ & 2.7519847616 & -3.5120486662 & -0.6400724955 \\
\hline & $\mathrm{H}$ & 1.8808874300 & -2.9826584498 & 0.8246216360 \\
\hline & $\mathrm{C}$ & 0.3576977247 & 1.9924542066 & 0.8965278089 \\
\hline & $\mathrm{H}$ & 1.3700658766 & 1.7431361025 & 1.2446793714 \\
\hline & $\mathrm{C}$ & -0.2401818412 & 3.0716784817 & 1.7769536856 \\
\hline & $\mathrm{H}$ & -1.2663262793 & 3.2838646086 & 1.4674143803 \\
\hline & $\mathrm{H}$ & -0.2445490375 & 2.7371363796 & 2.8159966454 \\
\hline & $\mathrm{H}$ & 0.3491884163 & 3.9899011939 & 1.7020844492 \\
\hline
\end{tabular}




\begin{tabular}{|c|c|c|c|c|}
\hline & $\mathrm{C}$ & 0.4737365281 & 2.3671425310 & -0.5744398120 \\
\hline & $\mathrm{H}$ & 1.0593985805 & 3.2818813340 & -0.6823297878 \\
\hline & $\mathrm{H}$ & 0.9306536614 & 1.5595867433 & -1.1469393446 \\
\hline & $\mathrm{Cl}$ & -1.1313097840 & 2.6718027314 & -1.3201967506 \\
\hline & $\mathrm{C}$ & -2.2224821501 & -1.4891429454 & 0.0326201127 \\
\hline & $\mathrm{H}$ & -2.6443400016 & -2.4974987104 & 0.0552968137 \\
\hline & $\mathrm{C}$ & -3.2607712307 & -0.4639263474 & 0.4640772713 \\
\hline & $\mathrm{H}$ & -4.1337753980 & -0.5293332468 & -0.1898558232 \\
\hline & $\mathrm{H}$ & -3.5707244276 & -0.6746352102 & 1.4902599736 \\
\hline & $\mathrm{H}$ & -2.8461400135 & 0.5458436586 & 0.4130269236 \\
\hline & $\mathrm{C}$ & -1.6881807412 & -1.2241189567 & -1.3693433604 \\
\hline & $\mathrm{H}$ & -1.3100506823 & -0.2126207165 & -1.5310754357 \\
\hline & $\mathrm{H}$ & -0.7576658440 & -1.9752229981 & -1.5160059181 \\
\hline & $\mathrm{Cl}$ & -2.8638712144 & -1.6237708706 & -2.6120344164 \\
\hline & $\mathrm{O}$ & 0.3229624087 & -2.7380863532 & -1.1891146511 \\
\hline \multirow[t]{22}{*}{$\mathrm{TS}_{\mathrm{R} 2-10-5}$} & $\mathrm{P}$ & 0.0626574477 & -0.3865978315 & 1.4310553895 \\
\hline & $\mathrm{O}$ & 0.8300898948 & -0.5816302715 & 2.6654664469 \\
\hline & $\mathrm{O}$ & -0.6834773949 & 1.0019798701 & 1.2119707978 \\
\hline & $\mathrm{O}$ & 0.8999106651 & -0.4727896949 & 0.0596843557 \\
\hline & $\mathrm{O}$ & -1.1315120592 & -1.4405179818 & 1.2717676030 \\
\hline & $\mathrm{C}$ & 2.8409173811 & -1.1974321684 & -1.1027807410 \\
\hline & $\mathrm{C}$ & 1.5007978929 & -1.6537198821 & -0.5027440683 \\
\hline & $\mathrm{C}$ & 1.7629750453 & -2.7323070455 & 0.5463650727 \\
\hline & $\mathrm{Cl}$ & 2.6665397394 & 0.1715654918 & -2.2323827528 \\
\hline & $\mathrm{H}$ & 3.2929307948 & -2.0256224404 & -1.6475452435 \\
\hline & $\mathrm{H}$ & 3.4891214663 & -0.8725346941 & -0.2878344684 \\
\hline & $\mathrm{H}$ & 2.3580849856 & -2.3365472718 & 1.3717202768 \\
\hline & $\mathrm{H}$ & 2.2835323762 & -3.5635466131 & 0.0662510330 \\
\hline & $\mathrm{H}$ & 0.8180305073 & -3.1015035054 & 0.9532535144 \\
\hline & $\mathrm{C}$ & -0.0818726261 & 2.1352220039 & 0.5505762894 \\
\hline & $\mathrm{H}$ & 1.0111766771 & 2.0418141460 & 0.5955558998 \\
\hline & $\mathrm{C}$ & -0.5377725558 & 3.3764423884 & 1.2900033590 \\
\hline & $\mathrm{H}$ & -1.6273344983 & 3.4494824086 & 1.2667402257 \\
\hline & $\mathrm{H}$ & -0.2070589830 & 3.3302031704 & 2.3292232436 \\
\hline & $\mathrm{H}$ & -0.1130159700 & 4.2684838997 & 0.8211085195 \\
\hline & $\mathrm{C}$ & -0.4553811617 & 2.1025253200 & -0.9239113069 \\
\hline & $\mathrm{H}$ & -0.0364634235 & 2.9726732656 & -1.4327200403 \\
\hline
\end{tabular}




$\begin{array}{lrrr}\mathrm{H} & -0.0831972919 & 1.1915437415 & -1.3944262138 \\ \mathrm{Cl} & -2.2348850817 & 2.1441504500 & -1.1691600234 \\ \mathrm{C} & -1.9120847560 & -1.4645412107 & 0.0604409002 \\ \mathrm{H} & -1.7035173758 & -0.5744433605 & -0.5459749220 \\ \mathrm{C} & -1.5543421145 & -2.7127780175 & -0.7035235305 \\ \mathrm{H} & -2.1147609536 & -2.8377033663 & -1.6309640903 \\ \mathrm{H} & -0.3994780276 & -2.5421998745 & -1.1143414422 \\ \mathrm{H} & -1.5391177966 & -3.6141130803 & -0.0864161874 \\ \mathrm{C} & -3.3625665628 & -1.3979682619 & 0.5292735047 \\ \mathrm{H} & -3.6033818827 & -2.2522162301 & 1.1639356953 \\ \mathrm{H} & -3.5157787775 & -0.4671773286 & 1.0768560842 \\ \mathrm{Cl} & -4.4828990428 & -1.4061789450 & -0.8642312556 \\ \mathrm{O} & 0.7367734423 & -2.0932885700 & -1.5588974449\end{array}$

$\begin{array}{rrrrr}\mathrm{TS}_{\mathrm{R} 2-10-6} & \mathrm{P} & 0.2009344009 & -0.5395804898 & 1.0784823928 \\ \mathrm{O} & 1.0114428195 & -0.8340718718 & 2.3414282122 \\ \mathrm{O} & -0.5948580365 & 0.8419609489 & 1.1571857812 \\ \mathrm{O} & 1.0714485977 & -0.5522661529 & -0.1777022035 \\ \mathrm{O} & -0.9911308069 & -1.5913833641 & 1.0178386136 \\ \mathrm{C} & 3.3347992037 & -0.8050171678 & 1.2251207324 \\ \mathrm{C} & 2.8027432982 & -1.4020768890 & -0.1361276809 \\ \mathrm{C} & 2.3316974892 & -2.8368866893 & -0.0341165361 \\ \mathrm{Cl} & 3.8012192856 & 0.8739048681 & 1.2210186873 \\ \mathrm{H} & 4.1621828149 & -1.4194009100 & 1.5877209803 \\ \mathrm{H} & 2.2519865839 & -0.8913045835 & 1.8975933986 \\ \mathrm{H} & 1.7366748324 & -2.9910124473 & 0.8673398528 \\ \mathrm{H} & 3.2189124288 & -3.4762009126 & -0.0093355229 \\ \mathrm{H} & 1.7430455870 & -3.0684344124 & -0.9217346870 \\ \mathrm{C} & 0.0033338451 & 2.0749795492 & 0.7074698827 \\ \mathrm{H} & 1.0899038233 & 2.0243373790 & 0.8612676855 \\ \mathrm{C} & -0.5971345416 & 3.1859350706 & 1.5444600789 \\ \mathrm{H} & -0.1796171035 & 4.1503159664 & 1.2417468216 \\ \mathrm{H} & -1.6813954259 & 3.2135527156 & 1.4125262059 \\ \mathrm{H} & -0.3719180941 & 3.0181595664 & 2.5993798246 \\ \mathrm{C} & -0.2171882710 & 2.2213519112 & -0.7908413432 \\ \mathrm{H} & 0.1732414151 & 3.1812955532 & -1.1334567970 \\ \mathrm{H} & 0.2716242789 & 1.4066266028 & -1.3251604636 \\ \mathrm{Cl} & -1.9616188848 & 2.1652174798 & -1.2165367323\end{array}$




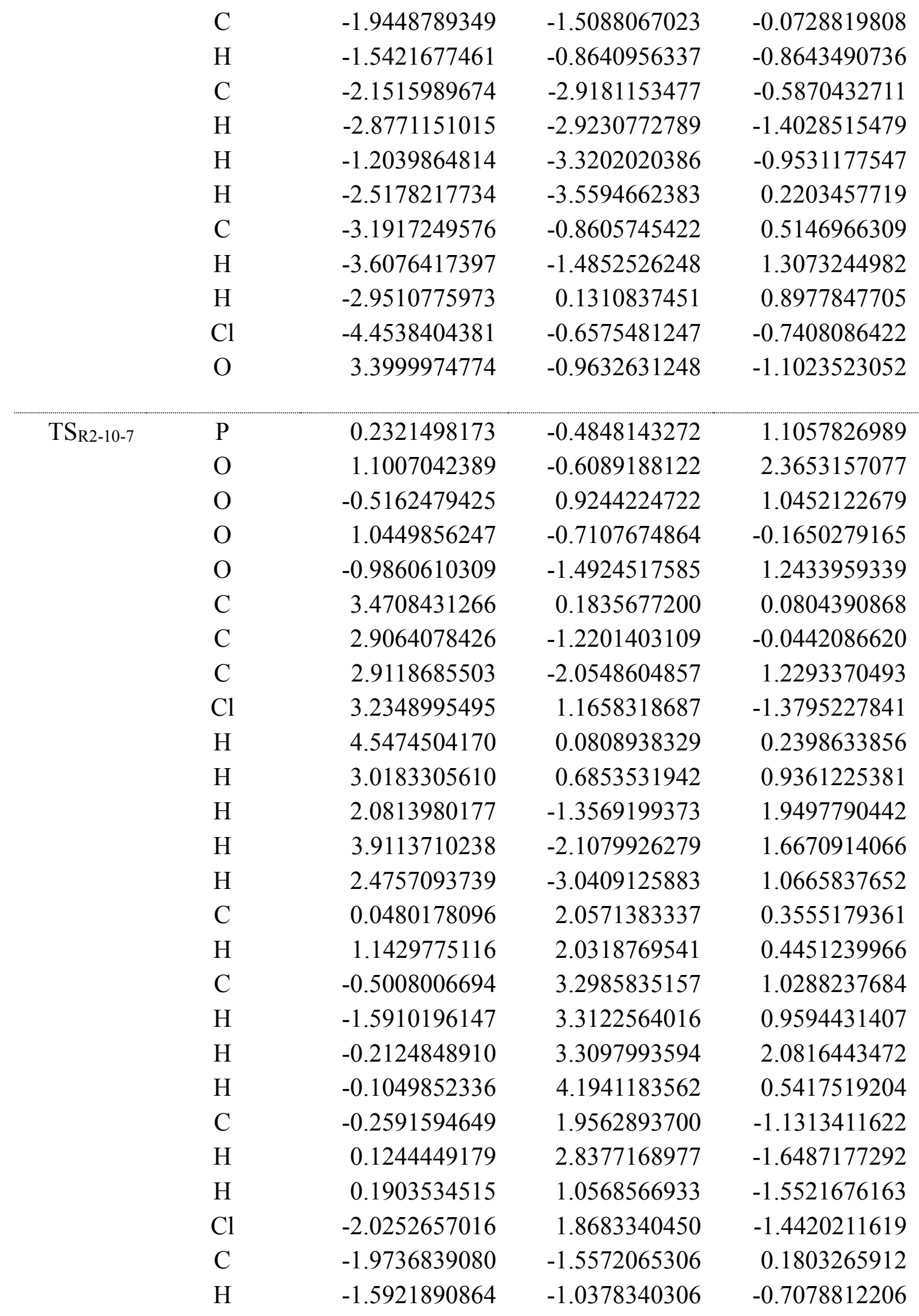




\begin{tabular}{|c|c|c|c|c|}
\hline & C & -2.2083085429 & -3.0221693784 & -0.1206866020 \\
\hline & $\mathrm{H}$ & -2.9595579072 & -3.1310884382 & -0.9052971594 \\
\hline & $\mathrm{H}$ & -1.2762628199 & -3.4837631863 & -0.4539520963 \\
\hline & $\mathrm{H}$ & -2.5563520297 & -3.5363756019 & 0.7803219279 \\
\hline & $\mathrm{C}$ & -3.1929276031 & -0.8158125433 & 0.7126850075 \\
\hline & $\mathrm{H}$ & -3.5822485091 & -1.3162300366 & 1.6011286504 \\
\hline & $\mathrm{H}$ & -2.9316985045 & 0.2176514923 & 0.9418332273 \\
\hline & $\mathrm{Cl}$ & -4.4999065726 & -0.7802430677 & -0.5119365024 \\
\hline & $\mathrm{O}$ & 3.1076805682 & -1.9398978793 & -1.0198843051 \\
\hline \multirow[t]{28}{*}{$\mathrm{TS}_{\mathrm{R} 2-10-8}$} & $\mathrm{P}$ & 0.1141698188 & -0.3179398466 & 1.3079780397 \\
\hline & $\mathrm{O}$ & 0.9621502443 & -0.4047426508 & 2.5066051683 \\
\hline & $\mathrm{O}$ & -0.7280776372 & 1.0022271347 & 1.0496176353 \\
\hline & $\mathrm{O}$ & 0.9478633088 & -0.4409273275 & -0.0725133933 \\
\hline & $\mathrm{O}$ & -1.0105888263 & -1.4363813351 & 1.2210783484 \\
\hline & $\mathrm{C}$ & 3.3023278719 & -0.1893006864 & 0.4101176916 \\
\hline & $\mathrm{C}$ & 2.2069126336 & -1.1076651352 & -0.0918893397 \\
\hline & $\mathrm{C}$ & 2.1940694331 & -2.5100664075 & 0.4493811962 \\
\hline & $\mathrm{Cl}$ & 3.3695663901 & 1.3122459646 & -0.5645452887 \\
\hline & $\mathrm{H}$ & 4.2666792540 & -0.6867045001 & 0.3063004714 \\
\hline & $\mathrm{H}$ & 3.1120965803 & 0.0829253069 & 1.4491213992 \\
\hline & $\mathrm{H}$ & 3.0874011558 & -2.8592346499 & 0.9600991841 \\
\hline & $\mathrm{H}$ & 2.3479040021 & -2.7387134649 & -0.8670024053 \\
\hline & $\mathrm{H}$ & 1.2331916184 & -2.9156723104 & 0.7590006348 \\
\hline & $\mathrm{C}$ & -0.1114008416 & 2.2350400085 & 0.6158276103 \\
\hline & $\mathrm{H}$ & 0.9597823063 & 2.2094038859 & 0.8589148446 \\
\hline & $\mathrm{C}$ & -0.7938163828 & 3.3616150861 & 1.3634426152 \\
\hline & $\mathrm{H}$ & -1.8635463970 & 3.3678090359 & 1.1412958567 \\
\hline & $\mathrm{H}$ & -0.6534323542 & 3.2314851165 & 2.4381916404 \\
\hline & $\mathrm{H}$ & -0.3648276625 & 4.3218480006 & 1.0640321676 \\
\hline & $\mathrm{C}$ & -0.2172285119 & 2.3193471672 & -0.8991666218 \\
\hline & $\mathrm{H}$ & 0.1726665666 & 3.2775271426 & -1.2474115179 \\
\hline & $\mathrm{H}$ & 0.3352330320 & 1.5047409659 & -1.3670862251 \\
\hline & $\mathrm{Cl}$ & -1.9214920715 & 2.1916982666 & -1.4505889623 \\
\hline & $\mathrm{C}$ & -1.9054540061 & -1.4770391100 & 0.0739565409 \\
\hline & $\mathrm{H}$ & -1.5071471130 & -0.8396240309 & -0.7242635922 \\
\hline & $\mathrm{C}$ & -1.9764823483 & -2.9196171247 & -0.3794724510 \\
\hline & $\mathrm{H}$ & -2.6631409163 & -3.0179053042 & -1.2224998578 \\
\hline
\end{tabular}




\begin{tabular}{crrrr} 
& $\mathrm{H}$ & -0.9863697460 & -3.2575604371 & -0.6960449817 \\
$\mathrm{H}$ & -2.3240770040 & -3.5548341815 & 0.4407487808 \\
$\mathrm{C}$ & -3.2251495784 & -0.8959849724 & 0.5635952808 \\
$\mathrm{H}$ & -3.6381217065 & -1.5104586523 & 1.3657610856 \\
$\mathrm{H}$ & -3.0769722375 & 0.1286945205 & 0.9061390357 \\
$\mathrm{Cl}$ & -4.4262950769 & -0.8483151882 & -0.7642487616 \\
$\mathrm{O}$ & 2.4357574720 & -1.5963197366 & -1.3878357496 \\
\hline $\mathrm{TS} \mathrm{R}_{\mathrm{N}-10-9}$ & & & \\
\hline $\mathrm{P}$ & 0.1376289515 & -0.2690843938 & 1.3037574106 \\
$\mathrm{O}$ & 0.9493432346 & -0.2837539858 & 2.5282275484 \\
$\mathrm{O}$ & -0.7694379700 & 1.0001902448 & 0.9974161063 \\
$\mathrm{O}$ & 1.0028883386 & -0.3746870711 & -0.0628104822 \\
$\mathrm{O}$ & -0.9396959033 & -1.4346828382 & 1.2145367208 \\
$\mathrm{C}$ & 3.4545925403 & -0.2732384735 & 0.1452632010 \\
$\mathrm{C}$ & 2.2168936113 & -1.1090660607 & -0.1352616274 \\
$\mathrm{C}$ & 2.1298588374 & -2.4723977618 & 0.5150233463 \\
$\mathrm{Cl}$ & 3.3151915393 & 1.3983347015 & 0.5827195525 \\
$\mathrm{H}$ & 3.5843841897 & -0.4264463057 & -1.1517281661 \\
$\mathrm{H}$ & 4.2869721066 & -0.7815598336 & 0.6279330415 \\
$\mathrm{H}$ & 1.9169357262 & -2.3858062309 & 1.5826023951 \\
$\mathrm{H}$ & 3.0784442012 & -2.9934886084 & 0.3708382989 \\
$\mathrm{H}$ & 1.3406958535 & -3.0500389551 & 0.0272906631 \\
$\mathrm{C}$ & -0.2308369599 & 2.3095640772 & 0.7289803890 \\
$\mathrm{H}$ & 0.7644728510 & 2.3837686669 & 1.1847554802 \\
$\mathrm{C}$ & -1.1713787343 & 3.3135044238 & 1.3646355978 \\
$\mathrm{H}$ & -2.1724956155 & 3.2153620060 & 0.9380123516 \\
$\mathrm{H}$ & -1.2230398548 & 3.1409835214 & 2.4413655360 \\
$\mathrm{H}$ & -0.8099534846 & 4.3301341366 & 1.1864357296 \\
$\mathrm{C}$ & -0.0586396631 & 2.4699485523 & -0.7744545986 \\
$\mathrm{H}$ & 0.2862494858 & 3.4800780839 & -1.0038210862 \\
$\mathrm{H}$ & 0.6483918829 & 1.7369333548 & -1.1620449488 \\
$\mathrm{Cl}$ & -1.6063440427 & 2.2125544018 & -1.6457394838 \\
$\mathrm{C}$ & -1.8408089876 & -1.5207273204 & 0.0775053777 \\
$\mathrm{H}$ & -1.4465949648 & -0.9158234053 & -0.7479086932 \\
$\mathrm{C}$ & -1.9244417252 & -2.9812026101 & -0.3136071921 \\
$\mathrm{H}$ & -2.6267235596 & -3.1113749159 & -1.1393643005 \\
$\mathrm{H}$ & -0.9437255622 & -3.3424786962 & -0.6302275397 \\
$\mathrm{H}$ & -2.2612476885 & -3.5772765100 & 0.5396512432 \\
& & & \\
& & $\mathrm{~S} 87$ & & \\
& & & & \\
& & & \\
& & & \\
& & &
\end{tabular}




\begin{tabular}{lrrr}
$\mathrm{C}$ & -3.1630028128 & -0.9267370357 & 0.5488551235 \\
$\mathrm{H}$ & -3.5785812882 & -1.5228387118 & 1.3636111226 \\
$\mathrm{H}$ & -3.0176870429 & 0.1046568288 & 0.8681562407 \\
$\mathrm{Cl}$ & -4.3604240765 & -0.9090334962 & -0.7833807943 \\
$\mathrm{O}$ & 2.5994708969 & -1.1829010996 & -1.4889698337 \\
\hline
\end{tabular}

293 


\section{References}

295 (1) Mellouki, A.; Wallington, T. J.; Chen, J., Atmospheric chemistry of oxygenated 296 volatile organic compounds: impacts on air quality and climate. Chem. Rev. 2015, 115, (10), 297 3984-4014.

298 (2) Suarez-Bertoa, R.; Picquet-Varrault, B.; Tamas, W.; Pangui, E.; Doussin, J. F., 299 Atmospheric fate of a series of carbonyl nitrates: photolysis frequencies and $\mathrm{OH}$-oxidation 300 rate constants. Environ. Sci. Technol. 2012, 46, (22), 12502-12509.

301 (3) Calvert, J. G.; Mellouki, A.; Orlando, J. J.; Pilling, M. J.; Wallington, T. J., The 302 Mechanisms of Atmospheric Oxidation of the Oxygenates. Oxford University Press: New 303 York, 2011.

304 (4) Chan, B.; Ball, G. E., A benchmark ab initio and dft study of the structure and binding 305 of methane in the sigma-alkane complex $\mathrm{CpRe}(\mathrm{CO})_{2}\left(\mathrm{CH}_{4}\right)$. J. Chem. Theory Comput. 2013, 306 9, (5), 2199-208.

307 (5) Atkinson, R.; Arey, J., Atmospheric degradation of volatile organic compounds. Chem. 308 Rev. 2003, 103, (1), 4605-4638.

309 (6) Boyd, A. A.; Flaud, P.-M.; Daugey, N.; Lesclaux, R., Rate constants for $\mathrm{RO}_{2}+\mathrm{HO}_{2}$ 310 reactions measured under a large excess of $\mathrm{HO}_{2}$. J. Phys. Chem. A 2003, 107, 818-821. 